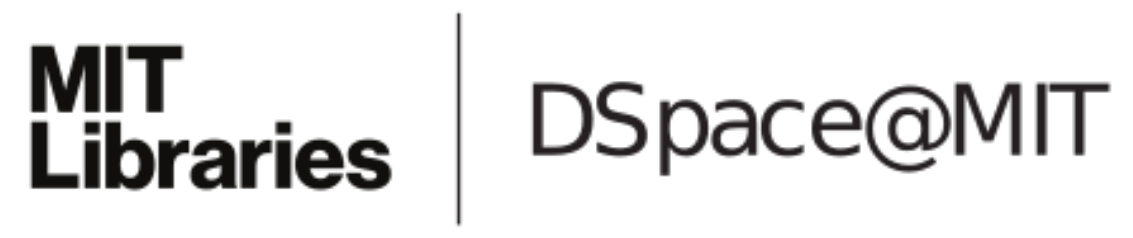

\author{
MIT Open Access Articles \\ Sourcing Flexibility, Spot Trading, \\ and Procurement Contract Structure
}

The MIT Faculty has made this article openly available. Please share how this access benefits you. Your story matters.

Citation: Pei, P. P.-E., D. Simchi-Levi, and T. I. Tunca. “Sourcing Flexibility, Spot Trading, and Procurement Contract Structure." Operations Research 59.3 (2011): 578-601.

As Published: http://dx.doi.org/10.1287/opre.1100.0905

Publisher: Institute for Operations Research and the Management Sciences

Persistent URL: http://hdl.handle.net/1721.1/69921

Version: Author's final manuscript: final author's manuscript post peer review, without publisher's formatting or copy editing

Terms of use: Creative Commons Attribution-Noncommercial-Share Alike 3.0 


\title{
Sourcing Flexibility, Spot Trading, and Procurement Contract Structure
}

\author{
Pamela Pen-Erh Pei* \\ Operations Research Center \\ M.I.T.
}

\author{
David Simchi-Levi ${ }^{\dagger}$ \\ Operations Research Center \\ M.I.T.
}

\author{
Tunay I. Tunca ${ }^{\ddagger \S}$ \\ Graduate School of Business \\ Stanford University
}

August 2007. This Revision: July, 2010

\begin{abstract}
We analyze the structure and pricing of option contracts for an industrial good in the presence of spot trading. We combine the analysis of spot trading and buyers' disparate private valuations for different suppliers' products, and jointly endogenize the determination of three major dimensions in contract design: (i) sales contracts versus options contracts; (ii) flat-price versus volume-dependent contracts; (iii) volume discounts versus volume premia. We build a model where a supplier of an industrial good transacts with a manufacturer who uses the supplier's product to produce an end good with an uncertain demand. We show that, consistent with industry observations, volume-dependent optimal sales contracts always demonstrate volume discounts (i.e., involve concave pricing). However, options are more complex agreements, and optimal option contracts can involve both volume discounts and volume premia. Three major contract structures commonly emerge in optimality. First, if the seller has a high discount rate relative to the buyer and the seller's production costs or the production capacity is low, the optimal contracts tend to be flat-price sales contracts. Second, when the seller has a relatively high discount rate compared to the buyer but production costs or production capacity is high, the optimal contracts are sales contracts with volume discounts. Third, if the buyer's discount rate is high relative to the seller's, then the optimal contracts tend to be volume-dependent options contracts and can involve both volume discounts and volume premia. However, when the seller's production capacity is sufficiently low, it is possible to observe flat price option contracts. We further provide links between production and spot market characteristics, contract design, and efficiency.
\end{abstract}

\footnotetext{
* Operations Research Center, 77 Massachusetts Avenue, Massachusetts Institute of Technology, Cambridge, MA 02137. e-mail: ppei@mit.edu

${ }^{\dagger}$ Operations Research Center, 77 Massachusetts Avenue, Massachusetts Institute of Technology, Cambridge, MA 02137. e-mail: dslevi.mit.edu

${ }^{\ddagger}$ Graduate School of Business, 518 Memorial Way, Stanford University, Stanford, CA 94305-5015. e-mail: tunca_tunay@gsb.stanford.edu

${ }^{\S}$ We thank Izak Duenyas (the Department Editor), the Associate Editor and three referees as well as Steve Graves, Evan Porteus, Jin Whang, Thomas Olavson from Hewlett-Packard Strategic Planning and Modeling group, and Venu Negali from Hewlett-Packard Procurement Risk Management Group for helpful comments and discussions.
} 


\section{Introduction}

As globalization of economies and advances in technology and communications increase the competitiveness of industries, companies find themselves increasingly under pressure to respond to ever faster changes in demand and supply. This stringent business environment forces companies to employ more flexible forms of procurement strategies and tools over and beyond the traditional, long-term, often close-relationship-based and rigid delivery agreements that historically dominated industrial procurement. As a consequence, there has been a rapid growth in the employment of two forms of procurement strategies in recent years: option contracts, and the utilization of spot trading to supplement existing contracts according to the resolution of uncertainties.

The first one of these two strategies, the use of flexible option contracts in industrial procurement, has drawn significant attention. In traditional sales contracts, a supplier of an industrial good agrees to deliver the procured goods to the buyer at the specified future date. On the other hand, option contracts in this context are agreements between a supplier and a buyer, where the buyer purchases the right to receive the delivery of the good from the supplier at a specified date at a predetermined exercise price. At the contract time, the buyer pays a reservation fee to purchase this right. At the time of delivery, depending on the resolution of uncertainties (such as the spot market price and consumer demand for the end product), the buyer decides the number of options to exercise up to the number reserved according to the contract agreement. Upon delivery by the supplier, the buyer pays an additional fee for the exercise of the options to the seller for only the exercised units. This provides flexibility and increased efficiency in risk sharing between the supply chain partners. Such contracts now have been used in a variety of industries and product categories, ranging from electricity, tools, and heavy equipment to specialized optical and electronic components.

Recent efforts, together with advancements in technology have also increased opportunities for utilizing industrial spot markets to complement existing contracts by allowing companies to connect with new trading partners and adjust to changing market conditions. In fact, today, in many industries in the United States and around the world, procurement is carried out as a mix of long-term agreements between suppliers and buyers, and spot purchases (see, e.g., Robertson 2002, Grey et al. 2005). However, there are significant downsides of spot trading industrial goods compared to procuring through contracts with known partners. First, there is naturally a spread between the buy and sell prices in these venues. That is, if one wants to sell a product, she has to accept a reduction in price compared to the price she would pay to buy the product. Second, and importantly, not all products are the same. Industrial goods from different suppliers have varying characteristics over a number of dimensions. As a consequence, an industrial buyer usually has different willingness-to-pay for different suppliers' products, valuing the products of their preferred suppliers, who are often their long term partners, at a premium. Such valuation differences stem from many dimensions such as compatibility, reliability, and match (see e.g., Donohue 2000, Levi et al. 2003). This means that spot purchases come with value losses due to product differences and transaction, 
adaptation, and compatibility costs (Williamson, 1981a,b, Malone et al. 1987, Baker et al. 2002). These inefficiencies create incentives to reduce reliance on spot markets for procurement and shift purchasing towards contracts with existing partners. Thus, the ability to adapt to trading with new suppliers becomes an important distinguishing characteristic. If a manufacturer is sufficiently flexible in product substitution, then his reliance on a given supplier is low, and he can switch between the products of long-term partners and new suppliers found on the spot market with relatively low value loss. In such a case, when signing long-term agreements, the buyer will have a strong position compared to the supplier. On the other hand, if a manufacturer's production process is not very flexible in terms of product substitution, procuring from new suppliers brings lower overall value, and hence he is highly reliant on his existing business partners.

As a result, the flexibility of a given manufacturer's production process becomes an important individual characteristic that determines the outside option of a manufacturer, and hence it is an important factor in the pricing of a procurement contract. As such, it is natural that the prices for sales or option contracts depend on the parties' respective information on the private valuation of the product of its long-term supplier compared to that of an outside provider. Therefore, at the contracting stage, a manufacturer would like to represent himself as minimally reliant on the supplier as possible. As we mentioned above, many factors combine to form the difference in the buyer's private valuation for the supplier's product compared to what he could buy from the spot, and determine a certain maximum willingness-to-pay (or, equivalently, cost of acquisition) for the buyer, which is normally private information to the buyer. Thus, when pricing the option contracts, the supplier has an informational disadvantage. Specifically, she has to determine the pricing terms for the option contracts without knowing the buyer's exact valuation for her product relative to those of other suppliers. The solution to this pricing problem is complex since it not only involves information asymmetry about the buyer's valuation, but also a simultaneous consideration of decision making on several dimensions, including the quantity of contracts to be agreed upon, as well as the exercising of option contracts based on the realization of uncertainties in spot market price and consumer demand. Further, the optimal exercise price for the contracts needs to be determined endogenously and simultaneously with the reservation price scheme. As a consequence, fundamental characteristics of the optimal contracts, such as volume-dependency structure and pricing, as well as the nature of the contracts (whether they will be options or sales contracts), are open for the supplier to decide, taking various components of uncertainty into account.

These observations lead to important research questions: How do non-linear pricing schemes for option contracts and spot market trading interact? What is the optimal joint option pricing scheme, including the reservation price schedule and the exercise price, in the presence of spot market trading? When is it optimal to employ flat (i.e., volume-independent) pricing? Alternatively, when a volume-dependent pricing scheme is employed, is it always optimal to offer volume discounts? When would a supplier offer options and when would she offer to simply sell to the buyer? How do market and industry characteristics such as production costs, production flexibility, spot price distribution, bid-ask spread for the spot price, and demand distribution affect contract characteristics such as the exercise price, reservation price, and the 
contracted quantity? In this paper, we study the answers to these questions. Specifically, our goal is threefold. First, starting with a general contract pricing point of view, we present the solution to the reservation pricing problem for the supplier for option agreements. In particular, we characterize the optimal general non-linear pricing scheme for the options on delivery of the industrial good with the presence of spot trading as an outside alternative for both parties, under information asymmetry on buyer's production flexibility and uncertainty on demand and spot price. Second, we determine the conditions under which it is optimal to sell the capacity or offer options, and the conditions under which it is optimal to offer volume-dependent pricing instead of flat pricing. Finally, we demonstrate the effects of market and industry characteristics on the specification of optimal contracts.

We show that, volume-dependent optimal sales contracts demonstrate volume discounts. However optimal option contracts can involve both volume discounts and volume premia. We show that three major contract structures commonly emerge in optimality: First, if the seller has a high discount rate relative to the buyer and the seller's marginal production cost or the production capacity is low, the optimal contracts tend to be flat-price sales contracts, i.e. the seller will make an offer to sell her capacity to the buyer at a lump-sum price. Second, when the seller has a relatively high discount rate compared to the buyer but production costs or production capacity is high, sales contracts with volume discounts are optimal. Third, if the buyer's discount rate is high relative to the seller's, then volume-dependent options contracts with both volume discounts and volume premia tend to be optimal. Exploring the effects of the industry and market characteristics on contracts, we find that increased average spot prices tend to increase the option exercise price, quantity contracted and reservation prices. Increased spot price variance, on the other hand, tends to decrease the exercise price and contracted quantity but increases the average reservation price. Finally, when the bid-ask spread in the spot market or production costs decrease, expected contracted quantity and average reservation price decrease.

The rest of this paper is organized as follows. Section 2 presents the literature review. Section 3 presents our model. Section 4 provides an analysis of the benchmark case where the buyer's preference valuation for the supplier's product is known to the supplier. Section 5 presents the design and characteristics of the optimal contracting schemes for the general case with buyer type uncertainty. Section 6 studies the effects of the industry and market characteristics on contract design. Section 7 provides a discussion of our model assumptions and potential extensions. Section 8 offers our concluding remarks. All proofs are given in the Online Supplement.

\section{Literature Review}

Supply chain contracting has received a considerable amount of attention in the literature in recent years. There are a large number of studies that explore a variety of supply chain contracting schemes. Cachon (2003) and Chen (2003) give comprehensive surveys of the literature in this area. Among many different contract structures studied in this literature, some examples are buy-back (Donohue 2000), "pay-to-delay" 
(Brown and Lee 1998), quantity flexibility (Tsay 1999), and revenue-sharing contracts (Cachon and Lariviere 2005). Option contracts for procurement are also among contract structures studied in the supply chain literature. Eppen and Iyer (1997) study option contracts (or "backup agreements") between catalog companies and manufacturers in the fashion industry. Examining data from the industry, they find that backup arrangements can have a substantial effect on expected profits and can increase contracted quantity. Barnes-Shuster et al. (2002) study channel coordination with option contacts, showing that coordination can be achieved through piece-wise linear exercise prices. They show, however, that to coordinate the channel through linear prices the supplier's individual rationality constraint has to be violated. Martinezde-Albeniz and Simchi-Levi (2008) study the bidding behavior in a market for supply option contracts with multiple suppliers and a single buyer. They show that in the Nash equilibrium of the bidding game, suppliers show clustering behavior. They also show that the loss of supply chain profit due to competition is, in general, at most $25 \%$ of the centralized supply chain profits.

A second main branch of the supply chain management literature that is closely related to our paper deals with spot trading in industrial goods. Kleindorfer and $\mathrm{Wu}$ (2003) provide a survey of the earlier literature in this area. Lee and Whang (2002) study the effect of a secondary market for excess inventory on a supply chain with a large number of buyers and a monopolistic supplier. They demonstrate that a secondary market increases allocative efficiency but may decrease the supplier's profits. Peleg et al. (2002) consider a multi-period setting with both long-term and spot purchases where unmet demand is carried to the next period. They identify the conditions under which each mode of procurement model is optimal. Dong and Durbin (2005) study industrial markets for surplus components and identify the conditions under which such a market would increase or decrease supplier and supply chain profits. Tunca and Zenios (2006) study the competition between relational contracts and auction markets in the presence of product quality differentiation among suppliers and determine the conditions under which long-term relational contracts can eliminate open auction markets and vice versa. Mendelson and Tunca (2007b) study sequential spot and long-term trading in a two-stage supply chain under asymmetric demand information. They define a concept for liquidity (or market impact factor) for industrial spot markets and demonstrate the important role it plays in supply chain efficiency and the generation of value and surplus in the supply chain. Shin and Tunca (2010) study market-based contracts that can be indexed to spot prices under diverse demand forecasts for multiple retailers and show that such contracts can aggregate the dispersed demand information and help coordinate the supply chain.

Wu et al. (2002) examine the interaction between capacity option contracts and spot trading. They explore a model with a single seller and multiple buyers, where the seller and the buyers first contract for capacity options and can later trade in the open spot market if it is desirable. They show that the buyers' optimal reservation level follows an index that combines the seller's reservation and execution costs. Wu and Kleindorfer (2005) utilize the same framework to examine a setting with multiple sellers with heterogenous technologies and a single buyer of the product. They characterize the equilibrium and explore its efficiency properties. Levi et al. (2003) introduce the notion of codifiability of the product and study the role of 
adaptation costs that an industrial buyer incurs when purchasing from the spot market. They show that codifiability and spot price distribution have significant effect on options contract pricing. Martinez-deAlbeniz and Simchi-Levi (2005a,b) employ a portfolio management approach to optimize supply option contracts in the presence of spot markets. They explore the mean-variance properties of supply option contract portfolios, and characterize the set of portfolios that a manufacturer must hold in order to achieve dominating mean-variance pairs. In a multi-period setting they also find the optimal replenishment policy for a portfolio of options.

In this paper, we aim to analyze the emergence of a variety of contract pricing structures. Earlier papers in the related literature start with a pre-set contract pricing form. For instance in Wu et al. (2002) the contracts are set as linear reservation price contracts. ${ }^{1}$ In contrast, we make the pricing structure endogenously emerge in optimality within a general class of contracts that encompasses commonly used contracting structures in practice. We jointly endogenize the determination of multiple dimensions of procurement contract specifications and find conditions under which the optimal contracts endogenously become sale contracts rather than option contracts (as suggested by $\mathrm{Wu}$ and Kleindorfer 2005 as an open research question), and under which optimal contracts will be volume-dependent instead of flat priced. Further, we explore the nature of volume dependency, and identify conditions, under which the optimal contracts demonstrate volume discounts and volume premia. These issues were not analyzed in the literature on industrial contracting with spot trading before. In addition, our paper introduces model features such as the buyer's true willingness-to-pay as his private information, and explores the role of difference between the discount rates of the buyer and the seller (which are almost always different in practice) on the contract structure. Finally, we also seek to understand the role of market and industry variables, such as spot price distribution and the statistical properties of informational asymmetry on the determination of contract structure and pricing. We explain the specifics of the interactions between these elements in detail in the next section, where we describe the model.

\section{The Model}

We study a two-stage supply chain. The supplier of an industrial intermediate good ("the seller" or " $S$ ") sells to a manufacturer ("the buyer" or " $B$ ", which can also be a retailer) who uses the intermediate good in his process to produce an end good. ${ }^{2}$ The demand for the end good, $D$, is uncertain and with a continuous distribution function $F_{D}$, density function $f_{D}$, and support $[\underline{D}, \bar{D}]$. The retail price for the end product is $p>0$. There are two time periods in the model. At time $t=1$, the buyer may reserve units from the seller, who is his preferred supplier, by purchasing $q \leq \bar{D}$ options, according to a price schedule $(R(q), w)$ the seller offers. The price schedule consists of a menu, $R(q)$, the reservation fee for $q$ options purchased, and the per-unit exercise fee, $w$. Each unit of option purchased gives the buyer the right to buy one unit

\footnotetext{
${ }^{1}$ Note that $\mathrm{Wu}$ et al. (2002) also focus on other dimensions of the setting such as the seller's capacity commitment decisions.

${ }^{2}$ As a language convention, we henceforth refer to the seller as "she" and the buyer as "he".
} 
of intermediate good from the seller at time $t=2$, at the exercise price $w \geq 0$. At time $t=1$, the buyer decides the amount of capacity to reserve, $q$, with the supplier and pays $R(q)$. The supplier who has a production capacity $K(\geq 0)$ then decides the number of units to produce $y \leq K$ at a unit production cost of $\beta>0$.

At time $t=2$, the consumer demand for the buyer's product, $D$, and the spot price for the intermediate good, $s$, are realized. The spot price, $s$, is uncertain at time $t=1$, with support $[\underline{s}, \bar{s}]$, where $0<\underline{s}<\bar{s}$. The distribution function $F_{s}$ for $s$ is continuous with density $f_{s}$. For tractability, we assume that $s$ and $D$ are independently distributed. ${ }^{3}$ We also assume that $s$ has increasing hazard rate; i.e., $f_{s}(s) / \bar{F}_{s}(s)$ is increasing, which is a commonly used weak assumption (see, e.g., Porteus 2002), and that $(d / d s) \log \left(f_{s}\right)$ is bounded on $[\underline{s}, \bar{s}]$, which ensures that $s$ distribution does not explode too fast over its support. As a regularity condition, we also assume that $\underline{s}>1 / f_{s}(\underline{s})$, which ensures a certain lower bound on the realization of the spot price. The first two conditions are satisfied by many common distributions on bounded support such as uniform, truncated normal, and truncated exponential. ${ }^{4}$ The third condition is on the support of the distribution and can also easily be simultaneously satisfied with the first two conditions by many distributions including the ones we mentioned above. For notational convenience, define $g_{s}(s) \triangleq \bar{F}_{s}(s) / f_{s}(s)$, which is decreasing in $s$. Observing $D$ and $s$, the buyer can exercise his options at the strike price $w$ up to the purchased amount, $q$. When the buyer places an order, exercising his options, the seller delivers the requested amount of the intermediate good. At the end of period 2, if either the seller or the buyer has any unused intermediate-good inventory, they can sell it to the spot market at price $(1-\phi) s$, where $0<\phi<1$ denotes the bid-ask spread at the spot market.

The buyer can also purchase the intermediate good from the spot market. However, normally, an industrial buyer has different valuations, or willingness-to-pay, for the products of different suppliers. Naturally, his valuation for a preferred supplier would be higher than that for outside suppliers. There are many reasons for this valuation difference, including such factors as the degree of fit of a given supplier's product, quality or reliability of that product, compatibility and specificity, the state of the buyer's production process, and the level and fit and integration of operating processes of the buyer and that supplier (see, e.g., Williamson 1981b, Malone et al. 1987, Hart and Moore 1988, Levi et al. 2003). Denote the difference in his willingness to pay for one unit of his preferred supplier's product and the product he procures from the open market by $\theta>0$. That is, every unit the buyer purchases on the spot comes with an additional reduction of payoff, or cost, of $\theta$. $\theta$ is private information to the buyer and a strong determinant of the buyer's dependence on his regular supplier, $S$. The supplier normally would not precisely know $\theta$. For his part, the buyer would protect information on the maximum extra amount he is willing to pay for the preferred supplier's product over that of others, since revealing it to the supplier would give her a strong advantage in contracting. From the seller's perspective, $\theta$ is a random variable with support $[\underline{\theta}, \bar{\theta}]$,

\footnotetext{
${ }^{3}$ See Section B in the Online Supplement, for an analysis with general correlation between the spot price and the demand realization for a special case of our model where introducing such correlation is tractable.

${ }^{4}$ Note that, granted that the standard definitions of heavy- and light-tailedness do not apply on bounded support, the increasing hazard rate condition is satisfied in our case in a wide spectrum of distributions on the support $[\underline{s}, \bar{s}]$ including "light-tailed" ones such as truncated normal, or "heavy-" or "fat-tailed" ones such as uniform.
} 
continuous distribution function $F_{\theta}$, and density function $f_{\theta}$. We assume that $\theta$ also has an increasing hazard rate, which means that $g_{\theta}(\theta) \triangleq \bar{F}_{\theta}(\theta) / f_{\theta}(\theta)$ is decreasing. As regularity conditions on the $s$ and $\theta$ distributions, we also assume $\underline{\theta} \geq \frac{1}{f_{\theta}(\underline{\theta})}$ and $f_{s}(\underline{s}) \in\left[\frac{1}{\phi \underline{s}+\underline{\theta}}, f_{\theta}(\underline{\theta})\right]$. The former maintains a balance in the low end of the $\theta$ distribution, and, together with the increasing hazard rate condition, can easily be simultaneously satisfied by many common distributions we mentioned above. The latter assumption is a technical assumption needed for tractability. It ensures the concavity of the seller's optimization problem and, in this sense, plays a similar role to the common single-crossing assumptions pricing and contracting literatures (see, e.g., Fudenberg and Tirole 1991, Mas-Colell et al. 1995). This assumption can again be easily satisfied by many common distributions on wide parameter ranges.

The supplier's discount rate between periods $t=1$ and $t=2$ is $r_{S}>0$; and the buyer's discount rate is $r_{B}>0$. In general, the two firms' discount rates would differ. There are a number of factors that determine a firm's discount rate. This rate reflects the firm's cost of capital, or borrowing rate, which, in turn, is affected by a number of characteristics such as firm size, and industry type and conditions. Usually, the larger the firm size, the lower its cost of capital and discount rate tend to be. For notational convenience, define $\rho=\left(1+r_{S}\right) /\left(1+r_{B}\right)$. Finally, for tractability, we also assume $p>\bar{s}+\bar{\theta}$, assuring that it is always profitable for the buyer to sell to the consumer market.

Having laid out the model description, before we move on to the analysis, let us highlight the types of contracts that can emerge. There are two main dimensions of contract characteristics here. First, the value of $w$ determines whether the contract is a sales or options contract. If $w=0$, the buyer will always exercise all the options he contracts with the supplier, which means that at $t=1$ the two parties are essentially entering into a sales agreement in which the supplier is effectively required to deliver all $q$ units to the buyer at time $t=2 .^{5}$ The same is also true when $0<w \leq s(1-\phi)$, in that the buyer in all cases will exercise his options, since he can sell them profitably to the spot market. In this case, the contracts will essentially be sales contracts with part of the payment deferred to $t=2$. However, if $w>s(1-\phi)$, the agreement is a true option contract; i.e., the seller may choose not to exercise the option with strictly positive probability. ${ }^{6}$ The second dimension of contract characteristic we will focus on will be volume dependency, which is captured by the nature of the pricing schedule $R(q)$. If the supplier offers a single lump-sum quantity to the buyer at a single price, i.e., if $R(q)$ is a single price-quantity point, that contract will be a flat-price contract. For instance, a contract where the seller offers the buyer a fixed capacity at a

\footnotetext{
${ }^{5}$ The sales contracts we have in our analysis can equivalently be called "forward contracts". In most cases in supplierbuyer relationships in the industry, however, "sales" or "purchases" are used commonly to refer to such contracts rather than "forward contracts". For consistency in terminology and conciseness, we use the term "sales contracts" throughout this paper.

${ }^{6}$ Forward and option contracts are also studied in the finance literature. From the perspective of that literature, such contracts are trading instruments that are priced in perfectly competitive markets often through arbitrage arguments. As such, for risk neutral traders, the expected returns for all such contracts are zero. As financial instruments, they can be used for a variety of purposes such as hedging, allocating capital across time, or speculative trading. See Duffie (1996) for a comprehensive treatment of forward and option contracts in the finance literature. In the industrial context we are studying however, the focus is on the agents who actually produce industrial goods at a certain cost, sell it to consumer markets for profit, have preferred trading partners whose products they value higher, and other production and supply chain specific features. In this environment, the supplier prices the forward (or sales) and options contracts to make profits from production and the value generation through the supply chain, and hence the performance of forward or option contracts take a different meaning than that in the context of the finance literature.
} 
fixed price is a flat-price contract. On the other hand, if the seller offers the buyer a pricing schedule that changes with the quantity contracted, i.e., if $R(q)$ varies with $q$, the contract is then a volume-dependent contract. For example, contracts with linear and non-linear $R(q)$, where the total reservation fee $R$ is monotonically increasing in $q$ are volume-dependent contracts. In our analysis, we will be studying the conditions for which the resulting contracts fall under each one of these types under the two dimensions of the characteristics we define above (see Section 5.3).

\section{Benchmark Case with Fixed Buyer Type}

Before we give the full analysis with random buyer type $\theta$, we first present the case of fixed $\theta$ as a simple benchmark. Here, as we will also do in the full solution, we first derive the optimal contract offer with a constant $w$, then optimize over $w$ to obtain the full optimal contract offer.

For optimal options contract design, the seller considers the buyer's optimal actions throughout the time horizon, given any feasible contract that she offers. The buyer's objective is to maximize his expected profits. Given the contract pricing and the exercise price, $(R(q, w), w)$, the buyer decides on the optimal capacity to reserve with the supplier, $q(R, w)$, at time $t=1$. At $t=2$, the buyer can exercise his options or purchase from the spot market or both. If there is a potential gain and the buyer has remaining options he does not use to satisfy consumer demand, he can also exercise his options to sell to the spot market. More specifically, at time $t=2$, given the values $(R(q, w), w)$ and $q(R, w)$ ( $q$ in short), when the two uncertain states $(s, D)$ are realized, the buyer optimally decides on three quantities: the number of options to exercise, the quantity to purchase from the spot market, and the quantity to sell to the spot market. The optimal decisions of the buyer on these three quantities are summarized in the following table:

\begin{tabular}{|c|c|c|c|}
\hline & Options exercised & Units purchased from the spot & Units sold to spot \\
\hline $0 \leq w<s(1-\phi)$ & $q$ & $(D-q)^{+}$ & $(q-D)^{+}$ \\
\hline$s(1-\phi) \leq w<s+\theta$ & $\min (D, q)$ & $(D-q)^{+}$ & 0 \\
\hline$s+\theta \leq w$ & 0 & $D$ & 0 \\
\hline
\end{tabular}

Define the buyer's expected discounted profit for given $q, w$ as $\pi_{B}(q, w, \theta)$. Then

$$
\begin{aligned}
\pi_{B}(q, w, \theta)=-R(q, w)+\frac{1}{1+} & r_{B}\left\{\left(p-E_{s}[\min (w, s+\theta)]\right) E_{D}[\min (D, q)]\right. \\
& \left.+\left(p-E_{s}[s]-\theta\right) E_{D}\left[(D-q)^{+}\right]+E_{s}\left[(s(1-\phi)-w)^{+}\right] E_{D}\left[(q-D)^{+}\right]\right\}
\end{aligned}
$$

In (1), the first expression accounts for the reservation cost of $q$ units. The second accounts for the buyer's discounted profit from selling up to $q$ units, where the buyer either exercises the options or buys from the spot market, whichever costs less. The third accounts for the buyer's discounted profit from selling above $q$ units, where the only choice for the buyer is to purchase from the spot market. The last expression accounts for the buyer's discounted profit from selling to the spot market when there are extra units available and 
the exercise cost is less than the spot's buy-back price. Note that (1), as the rest of the analysis in this section, utilizes the assumption of independence between $D$ and $s$ (which is relaxed in Section B in the online supplement for this benchmark case).

Let $\hat{\pi}_{B}(q, w, \theta)$ denote the buyer's net benefit from the option contract; i.e., the difference in his expected profits between the case he buys the option contract and the case he solely relies on spot trading. That is,

$$
\hat{\pi}_{B}(q, w, \theta)=\pi_{B}(q, w)-\frac{1}{1+r_{B}} E_{D}[D]\left(p-E_{s}[s]-\theta\right) .
$$

Combining (1) and (2), we then have

$$
\begin{aligned}
\hat{\pi}_{B}(q, w, \theta)=-R(q, w)+\frac{1}{1+r_{B}} E_{D}[\min (D, q)] & \int_{w-\theta}^{\bar{s}}(s+\theta-w) d F_{s}(s) \\
& +\frac{1}{1+r_{B}} E_{D}\left[(q-D)^{+}\right] \int_{\frac{w}{1-\phi}}^{\bar{s}}(s(1-\phi)-w) d F_{s}(s) .
\end{aligned}
$$

For future reference, denote $\varphi(q, w, \theta)=\left(1+r_{B}\right)\left(\hat{\pi}_{B}(q, w, \theta)+R(q, w)\right)$. Next, consider the seller's problem. For a given $w \geq 0$, the seller's expected profits can be written as

$$
\pi_{S}(q, w, \theta)=R(q, w)+V(q, w, \theta)
$$

where

$$
\begin{aligned}
V(q, w, \theta)=\frac{1}{1+r_{S}}\left\{\int_{\underline{s}}^{w-\theta} s(1-\phi) d F_{s}(s)\right. & +\int_{w-\theta}^{\frac{w}{1-\phi}}\left(w E_{D}[\min (D, q)]+s(1-\phi) E_{D}\left[(q-D)^{+}\right]\right) d F_{s}(s) \\
& \left.+w q \bar{F}_{s}\left(\frac{w}{1-\phi}\right)\right\}-\beta q+\left(\frac{E[s](1-\phi)}{1+r_{S}}-\beta\right)^{+}(K-q) .
\end{aligned}
$$

In (5), the first term represents the seller's discounted expected revenue when the spot price is sufficiently low that the buyer does not exercise any options, and the seller sells all she produces to the spot market. The second term in (5) is the seller's discounted expected revenue for the case where the buyer exercises some of the options, and the supplier sells the remaining to the spot market. The third term accounts for the seller's discounted expected revenue when the buyer exercises all options contracted. The fourth term represents the production costs for the units produced under the contracts. Finally, the last term is the seller's expected discounted profit from producing to directly sell to the spot market, which is positive only if $\beta<(E[s](1-\phi)) /\left(1+r_{S}\right)$. Notice that if the seller does not engage in contracting with the buyer at all, her expected profit is $\left(\frac{E[s](1-\phi)}{1+r_{S}}-\beta\right)^{+} \cdot K$. Define $\hat{\pi}_{S}(q, w)=\pi_{S}(q, w)-\left(\frac{E[s](1-\phi)}{1+r_{S}}-\beta\right)^{+} \cdot K$. The seller's problem can then be formulated as

$$
\begin{aligned}
\max _{R(\cdot, w)} & \pi_{S}(q, w, \theta) \equiv R(q, w)+V(q, w, \theta)=-\hat{\pi}_{B}(q, w, \theta)+\frac{\varphi(q, w)}{1+r_{B}}+V(q, w) \\
\text { subject to } & q=\arg \max _{\xi \geq 0} \hat{\pi}_{B}(\xi, w, \theta), \\
& \hat{\pi}_{B}(q, w, \theta) \geq 0, \hat{\pi}_{S}(q, w) \geq 0 .
\end{aligned}
$$


In (6), the first constraint is the Incentive Compatibility (IC) constraint for the buyer; i.e., it guarantees that the buyer chooses the best contract available to him. The second and third constraints are the Individual Rationality (IR) constraints for the buyer and the seller respectively, guaranteeing that neither is worse off by entering the contract (note that $\xi$ is a dummy variable for selection of $q$ in the optimization). The next proposition gives the optimal contract offer for the buyer for a fixed exercise price $w .^{7}$

\section{Proposition 1}

(i) For a given $w \geq 0$, define $\left.G(w)=E_{s}\left[(s(1-\phi)-w)^{+}\right]\right)$. In the optimal contract, the supplier offers the quantity

$$
q^{*}(w, \theta)=\min \left(K, \bar{F}_{D}^{-1}\left(\frac{\left(\left(1+r_{S}\right) \beta-E[s](1-\phi)\right)^{+}-(\rho-1) G(w)}{\int_{w-\theta}^{\bar{s}}(\phi s+\rho \theta) d F_{s}(s)+(\rho-1) E\left[(s-w) 1_{\{s+\theta \geq w\}}\right]-(\rho-1) G(w)}\right)\right),
$$

to the buyer at the price

$$
\begin{aligned}
R\left(q^{*}(w, \theta), w, \theta\right)=\frac{1}{1+r_{B}}\left(E_{D}\left[\min \left(D, q^{*}(w, \theta)\right)\right] \int_{w-\theta}^{\bar{s}}(s+\theta-w) d F_{s}(s)\right. & \\
& \left.+E_{D}\left[\left(q^{*}(w, \theta)-D\right)^{+}\right] \int_{\frac{w}{1-\phi}}^{\bar{s}}(s(1-\phi)-w) d F_{s}(s)\right) .
\end{aligned}
$$

(ii) By the definition given in (4), the supplier's problem for determining the optimal exercise price $w^{*}$ can be written as

$$
\max _{w \geq 0} \pi_{S}(q, w, \theta) \equiv \max _{w \geq 0} R\left(q^{*}(w, \theta), w, \theta\right)+V\left(q^{*}(w, \theta), w, \theta\right)
$$

Then,

(a) When $r_{B}<r_{S}, w^{*}=0$.

(b) When $r_{B}=r_{S}$, there is a continuum of optimal contracts. In particular, any $w^{*} \in[0, \underline{s}+\theta]$ is optimal.

(c) When $r_{B}>r_{S}, w^{*} \in[\underline{s}+\theta, \bar{s}+\theta]$. Further, there exists a $\delta_{r}>0$ such that when $r_{S}<r_{B}<r_{S}+\delta_{r}$, $\pi_{S}$ is non-monotonic in $w$ and $w^{*}<\bar{s}+\theta$.

As can be seen in part (i) of Proposition 1, the optimal contract quantity is either $K$ or carries the characteristics of a critical fractile solution. Specifically, critical fractile solution has the general structure

$$
\text { Optimal Quantity }=\bar{F}_{D}^{-1}\left(\frac{\text { Unit Production Cost }- \text { Unit Salvage Value }}{\text { Unit Sales Price }- \text { Unit Salvage Value }}\right)
$$

Parallel to (10), in the fractile expression in (7), the first term in the numerator $\left(\left(1+r_{S}\right) \beta-E[s](1-\phi)\right)^{+}$ is the production cost minus the expected sales price (in future dollars for the seller), provided that this

\footnotetext{
${ }^{7}$ The notation $1_{\{\}}$represents the indicator function.
} 
difference is positive. In other words, it is the amount that the seller needs to be compensated in order to be coaxed into producing one unit. In the second term, the expression $E_{s}\left[(s(1-\phi)-w)^{+}\right]$captures the expected salvage value for contracting one additional unit for the buyer. Given that the selling price $s(1-\phi)$ in the spot market is higher than the exercise price of the options, the buyer can salvage any unused quantity and obtain an amount $s(1-\phi)-w$ per unit. (The multiplier $\rho-1$ adjusts for the discount rate difference between the buyer and the seller.) The denominator of the fractile expression also reflects a parallel to (10). The first term captures the supply chain's expected savings from each unit contracted and exercised: When the spot price is high enough for the buyer to exercise options at price $w$, the unit loss, $\phi s$, from the resale of the good to the spot market due to the bid-ask spread as well as the buyer's loss, $\theta$ (adjusted for the discount rate difference between the buyer and the seller), due to purchasing from a non-preferred supplier on the spot market are avoided. In addition, given the spot price is sufficiently high, by exercising his options, the buyer also avoids spending an excess of $s-w$ in the spot market as captured by the second term. That is, the first two terms in the denominator combined reflect (discount adjusted) the expected benefit for the buyer of contracting one unit with the supplier. Finally, the last term $(\rho-1) G(w)$ again reflects the buyer's expected salvage value for the contacted units unsold to the spot market.

An important observation from part (i) of Proposition 1 is that if $\theta$ is fixed, the contract offer is fixed and is not volume dependent. This is because the supplier knows the buyer's preferences and offers the precise bundle that extracts all expected surplus the buyer makes from the contract in terms of reservation fees: the first term in (8) corresponds to the buyer's expected discounted savings from exercising his options instead of buying the intermediate good from the spot; and the second term corresponds to his surplus from exercising and selling any potential unused option to the spot market provided that the spot selling price $(s(1-\phi))$ exceeds the option exercise price $(w)$. As we will see in Section 5, when one takes into account the uncertainty in the buyer's preferences, in the optimal contract, in many cases, the buyer will have to tie the price paid to the quantity of the options purchased, resulting in volume-dependent pricing as commonly observed in practice. This will also result in the supplier's leaving positive expected surplus to the buyer.

When optimizing $w$, the supplier needs to consider the trade-off between collecting revenues now (in the form of reservation fees, $\left.R\left(q^{*}(w, \theta), w, \theta\right)\right)$ and collecting revenues in the future (in the form of exercise fees as they affect $V\left(q^{*}(w, \theta), w, \theta\right)$, where $V$ is as defined in (5)). Therefore, an important factor affecting the supplier's decision will be her discount rate. However, the supplier is interacting with the buyer in signing the contracts, and hence she has to take the buyer's preferences into account when determining the optimal exercise price. The final outcome reflects a combination of the preferences of both parties.

In the optimal contracts, the sales versus options decision hinges on the relative magnitudes of the seller's and the buyer's discount rates. As part (ii)(a) of Proposition 1 states, when the seller's discount rate is higher than the buyer's, the seller's optimal action is to offer a sales contract. Given the seller has a higher discount rate than the buyer, it becomes an efficient fee collection arrangement to front- 
load the payment from the buyer to the seller by setting the exercise price to zero and maximizing the reservation fee. That is, the discounted value of the revenue increase from increased exercise price cannot compensate for the loss in the highest expected reservation price the seller can get in the corresponding optimal contracts.

For the "knife-edge" case when the seller and the buyer have the same discount rate, there is a continuum of optimal exercise prices for the supplier as stated in part (ii)(b) of Proposition 1 . As long as $0 \leq w \leq \underline{s}+\theta$, i.e., as long as it is certain that the buyer will always exercise all units he contracted for, the supplier can compensate exactly for the expected reduction in the optimal reservation fee resulting from the increased exercise price she can get from the buyer. This is because the present value of the expected revenue increase in time $t=2$ is equal to the expected minimum reduction in reservation fee each type of buyer requires for the reduction in the value of option. Thus, in the optimal contracts, for the range $0 \leq w \leq \underline{s}+\underline{\theta}$, increasing $w$ has the effect of transferring revenue from future to present at the same rate, and hence the supplier's profit is constant in $w$ over this range. However, when the exercise price increases further, there will be strictly positive probability that the buyer will not exercise some of the units he contracted for, and for $w>\underline{s}+\theta$, the seller's profit will strictly decrease compared to the optimal level. ${ }^{8}$

When $r_{B}>r_{S}$, seller's profit can be non-monotonic in $w$. Given the buyer has a higher discount rate than the seller, in the optimal reservation price scheme, the seller can have a relatively steep increase in the exercise price with a small decrease in the reservation price. For low $w$ values, the seller can keep the present value of the difference as her profit, and increase her profits with increased exercise price. However, as $w$ increases, the value of the options start decreasing rapidly for the buyer. In this case, the supplier has to offer large discounts in reservation fees in order to sell the options. Consequently, beyond a certain point, increased exercise price decreases the supplier's overall profit, and the resulting profit curve is maximized at an interior unit exercise price, on $[\underline{s}+\theta, \bar{s}+\theta]$, as stated in part (ii)(c) of Proposition 1.

\section{Optimal Contract Design and Characteristics with Buyer Type Un- certainty}

We now present the contract solution for the general case with supplier uncertainty on the buyer type. We again start with the optimal design of the contract offered by the seller to the buyer for a given exercise price, $w$. We then provide the solution of the problem of determining the optimal exercise price $w^{*}$, deriving

\footnotetext{
${ }^{8}$ Note that in $\mathrm{Wu}$ et al. (2002), it is stated that the optimal exercise price for the model they examine (in which the buyer and the seller have equal discount rates) is the marginal production cost for the supplier, while in our model, we find that there is a continuum of exercise prices that are optimal for the buyer. Keeping in mind that the two models have a number of differences, it is worth pointing out the optimality of a unique exercise price in Wu et al. (2002) depends on the strict positivity of the density function of the spot price distribution on the relevant range. For instance, if the lower bound of support for the spot distribution in Wu et al. (2002) is higher than the marginal cost, a continuum of exercise prices from zero to the lower bound of the support of the spot price distribution would be optimal in that case as well. The key for both cases is that (given the buyer and seller discount rates are the same), as long as the seller guarantees that the buyer will exercise all the options purchased, she only cares about the total payment per option and can freely switch the payment back and forth between the reservation and exercise fees.
} 
the full contract characterization.

\subsection{Seller's Optimal Reservation Price Schedule for Fixed Exercise Price}

Given the optimal exercise and purchasing strategies of the buyer as discussed in Section 4, for any given exercise price $w \geq 0$, the seller's problem is to find the optimal reservation price schedule, $R(q(w, \theta), w)$, maximizing her expected profits, $\pi_{S}(w)$, where $q(w, \theta)$ is the reservation quantity for a type $\theta$ buyer. Note that there are two equivalent ways of representing the reservation price schedule. First, one can express the reservation price as a function of the quantity contracted, i.e., $R(q)$. Equivalently, noticing that each type $\theta$ buyer will pick a certain quantity $q$, which will correspond to a certain reservation fee $R$, one can write the reservation price schedule as $(R(\theta), q(\theta))$. As it is usually done in the literature (see, e.g., Myerson 1981) we will use the latter representation in the derivation of the solution. One can then map the reservation fee $R(\theta)$ with the corresponding quantity $q(\theta)$ for each $\theta$ to obtain $R(q)$.

We can write

$$
\pi_{S}(w)=E_{\theta}[R(q(w, \theta), w)+V(q(w, \theta), w, \theta)],
$$

where $V(q, w, \theta)$ is as defined in Section 4. Specifically, the optimal contract has to make sure that the quantity purchased by a type $\theta$ buyer is indeed his optimal quantity given the contract terms. In addition, no buyer type, nor the seller, should have negative expected gains upon contract agreement. Given this, the seller's problem can now be formulated as

$$
\begin{aligned}
\max _{R(\cdot, w)} & \int_{\underline{\theta}}^{\bar{\theta}}[R(q(w, \theta), w)+V(q(w, \theta), w, \theta)] d F_{\theta}(\theta) \\
\text { s.t. } & q(w, \theta)=\arg \max _{\xi \geq 0}\left[\hat{\pi}_{B}(\xi, w, \theta)\right], \forall \theta, \\
& \hat{\pi}_{B}(q(w, \theta), w, \theta) \geq 0, \forall \theta, \\
& \int_{\underline{\theta}}^{\bar{\theta}}[R(q(w, \theta), w)+V(q(w, \theta), w, \theta)] d F_{\theta}(\theta) \geq\left(\frac{E[s](1-\phi)}{1+r_{S}}-\beta\right)^{+} \cdot K, \\
& 0 \leq q(w, \theta) \leq K, \forall \theta .
\end{aligned}
$$

In (12), parallel to (6) in Section 4, the first constraint is the Incentive Compatibility (IC) constraint for the buyer, guaranteeing that the quantity purchased by each type of buyer is the best option for that type, and the second and third constraints are the Individual Rationality (IR) constraints for the buyer and the seller, respectively, guaranteeing that each one is better off participating in the contract than not participating. The final constraint states the capacity limit on the seller's production.

We first start with the case $K \geq \bar{D}$, and later we extend the solution to the case with $K<\bar{D}$. Presenting the solution to (12) for the former case, we first identify a set of conditions that determine whether the seller chooses a flat-price schedule or a volume-dependent pricing scheme. The following lemma will be helpful in this characterization. 
Lemma 1 For $w \in[0, \bar{s}(1-\phi)]$, let $G(w)$ be as defined in Proposition 1. If $r_{S}>r_{B}$, and $\beta \leq E[s](1-$ $\phi)\left(1+r_{B}\right)^{-1}$, then there exists a unique $\tilde{w}_{c} \in[0, \bar{s}(1-\phi)]$ such that $G\left(\tilde{w}_{c}\right)=\left(\left(1+r_{S}\right) \beta-E[s](1-\right.$ $\phi))^{+} /(\rho-1)$.

In certain cases, specifically when the number of options contracted exceeds consumer demand, and the option exercise price is lower than the bid price at the spot market, the buyer can exercise the remaining options to sell to the spot market. The expected per-unit profit the buyer gets from such a transaction is $G(w)$ as defined in Lemma (1). In establishing a certain mathematical property of $G(w)$, Lemma 1 is critical for the conditions that yield to volume-dependent pricing in option contracts, which we give next.

Definition We say the flat-price conditions are satisfied when all of the following three conditions are satisfied: $($ i $) r_{S}>r_{B}$; (ii) $\beta \leq E[s](1-\phi)\left(1+r_{B}\right)^{-1}$; and $($ iii $) w \leq \tilde{w}_{c}$ where $\tilde{w}_{c}$ is as defined in Lemma 1.

The flat-price conditions play a critical role in determining the nature of procurement contracts. They essentially require the supplier discount the future payments more than the buyer and that the unit production cost and exercise price of the contract are sufficiently low. Specifically, note that for any $\tilde{w}_{c} \geq 0, G\left(\tilde{w}_{c}\right)=\left(\left(1+r_{S}\right) \beta-E[s](1-\phi)\right) /(\rho-1)$ is equivalent to the condition

$$
\frac{G\left(\tilde{w}_{c}\right)}{1+r_{B}}=\left(\beta-\frac{1}{1+r_{S}} E[s](1-\phi)\right)^{+}-\frac{1}{1+r_{S}} G\left(\tilde{w}_{c}\right)
$$

The left-hand side of equation (13) is the buyer's expected discounted benefit from having one remaining option, with an exercise price of $\tilde{w}_{c}$, in excess of the consumer demand; i.e., to sell to the spot market. The right-hand side is the seller's opportunity cost of committing to one unit of option at exercise price $\tilde{w}_{c}$. Specifically, it is the present value of cost of producing the unit, less the expected amount she can get from the spot market for that unit (provided that the former is larger than the latter), adjusted for the expected exercise price, $\tilde{w}_{c}$, she will get from the buyer for that unit. Combining this intuition with the fact that $G$ is decreasing in $w$, the flat price condition (iii) implies gains from trade between the buyer and the seller from each unit committed at exercise price $w$, and by Lemma 1, flat price conditions $(i)$ and (ii) guarantee the existence of such $w$.

Given this intuition, we can now present the optimal contract offer for a fixed exercise price.

\section{Proposition 2}

(i) If the flat price conditions are satisfied, the optimal contracts are not volume-dependent. Rather, in the optimal offer, the reservation price is constant and given by $\varphi(\bar{D}, w, \underline{\theta}) /\left(1+r_{B}\right)$, and $q^{*}(w, \theta)=\bar{D}$ for all $\theta \in[\underline{\theta}, \bar{\theta}]$.

(ii) Suppose the flat price conditions are not satisfied. Then given $0<\rho<\bar{\rho}$, where $\bar{\rho} \triangleq 1+\frac{\phi}{\sup _{s \in[s, s]}\left|\frac{d g_{s}(s)}{d s}\right|}$, the optimal reservation price schedule for the seller is volume-dependent. Specifically, the optimal 
quantity ordered for type $\theta$ buyer is

$$
q^{*}(w, \theta)=\bar{F}_{D}^{-1}\left(\frac{\left(\left(1+r_{S}\right) \beta-E[s](1-\phi)\right)^{+}-(\rho-1) G(w)}{\eta(w, \theta)-(\rho-1) G(w)}\right)
$$

where

$$
\eta(w, \theta)=\int_{w-\theta}^{\bar{s}}\left(\phi s+\rho \theta-g_{\theta}(\theta)\right) d F_{s}(s)+(\rho-1) \int_{w-\theta}^{\bar{s}}\left(s-g_{\theta}(\theta)-w\right) d F_{s}(s),
$$

and $G$ is as defined in Lemma 1. The optimal total reservation fee paid by a type $\theta$ buyer is

$$
R\left(q^{*}(w, \theta), w\right)=\frac{1}{1+r_{B}}\left(\varphi\left(q^{*}(w, \theta), w, \theta\right)-\left.\int_{\underline{\theta}}^{\theta} \frac{\partial \varphi(q, w, a)}{\partial a}\right|_{q=q^{*}(w, a)} d a\right) .
$$

Further, $q^{*}(w, \theta)<\bar{D}$ and $q^{*}(w, \theta)$ is monotonically increasing in $\theta$ on $\theta \in[\underline{\theta}, \bar{\theta}]$.

As part $(i)$ of Proposition 2 states, when the seller has a higher discount rate and the production costs and the option exercise price $w$ are sufficiently low, the seller prefers to offer a flat-price contract and the buyer, independent of his type, $\theta$, chooses to purchase up to the seller's production capacity. However, when any of these three conditions are not satisfied, the seller finds it optimal to employ volume-dependent pricing. Note that $g_{s}(s)$ is monotonically decreasing in $s$, finite at $\underline{s}$, and $d \log \left(f_{s}(s)\right) / d s$ is bounded on $[\underline{s}, \bar{s}]$. Therefore $\left|\frac{d g_{s}(s)}{d s}\right|<\infty$, for all $s \in[\underline{s}, \bar{s}]$, and hence $\bar{\rho}>1 .^{9}$ For conciseness in exposition, in the remaining propositions, we will assume $\rho<\bar{\rho}$.

Part (ii) of Proposition 2 gives the optimal contract structure when the optimal contract is volume dependent. As in the case for fixed $\theta$, which was given in Proposition 1, the quantity for any given $\theta \in[\underline{\theta}, \bar{\theta}]$ is a critical fractile solution. The structure of the critical fractile for each $\theta$ closely parallels the structure for the fixed $\theta$ case discussed in Section 4, with a difference in the denominator: The upside of contracting each unit, captured by $\eta(w, \theta)$ in Proposition 2, is modified by the adjustment terms $g_{\theta}(\theta)$, due to the supplier's uncertainty about $\theta$ on the contracted quantity. Notice that, since $g_{\theta}(\theta) \geq 0$ for all $\theta \in[\underline{\theta}, \bar{\theta}]$, the net effect of the information asymmetry is reduced quantity contracted for each buyer type, $\theta$.

We next extend the result of Proposition 2 to the low capacity $(K \leq \bar{D})$ case. Note that in this case three possible general reservation price schedules may emerge. $R(q(\theta, w), w)$ can be (i) flat, i.e., nondependent on $\theta$; (ii) fully volume dependent, i.e., monotonically increasing on the entire range of $\theta$; (iii) mixed-menu, i.e., first monotonically increasing then flat on $[\underline{\theta}, \bar{\theta}]$.

Proposition 3 Suppose $K<\bar{D}$. The optimal reservation quantity schedule satisfies $q^{*}(w, \theta, K)=$

\footnotetext{
${ }^{9}$ The proposition is valid for $\rho<\bar{\rho}$ only, and tractability is lost for higher $\rho$ values. However, the region $\rho<\bar{\rho}$, for $\bar{\rho}$ as given in the proposition normally covers the practically relevant range. First, $\rho$ is normally around (but almost never equal to) one since $r_{S}$ and $r_{B}$, the discount rates for the two agents tend to be relatively close and between 0 to $10 \%$, meaning $\rho$ is often less than 1.1. This is because firms can typically borrow from banks at the ongoing "Bank Prime Loan Rate" plus a few percentage points, and large firms can even often borrow at rates under the prime loan rate (Berk and DeMarzo 2007). The historical prime rates (with exceptions for brief periods) tend to be under $10 \%$ (see, e.g., U.S.F.R.B. 2008).
} 
$\min \left\{K, q^{*}(w, \theta)\right\}$, where $q^{*}(w, \theta)$ is as defined in (14). The optimal price schedule can be obtained by plugging $q^{*}(w, \theta, K)$ in (16). Further,

(i) If $\eta(w, \underline{\theta}) \bar{F}_{D}(K) \geq\left(\left(1+r_{S}\right) \beta-E[s](1-\phi)\right)^{+}-(\rho-1) F_{D}(K) G(w)$, the contract is a flat price offer at $q^{*}(w, \theta)=K$.

(ii) If $\eta(w, \bar{\theta}) \bar{F}_{D}(K) \leq\left(\left(1+r_{S}\right) \beta-E[s](1-\phi)\right)^{+}-(\rho-1) F_{D}(K) G(w)$, the contract offer is fully volume dependent, and $q^{*}(w, \theta)$ is increasing for the entire support $[\underline{\theta}, \bar{\theta}]$.

(iii) Otherwise, there exists $\theta^{c}(w) \in[\underline{\theta}, \bar{\theta}]$ such that $\eta\left(w, \theta^{c}(w)\right) \bar{F}_{D}(K)=\left(\left(1+r_{S}\right) \beta-E[s](1-\phi)\right)^{+}$ $-(\rho-1) F_{D}(K) G(w)$, and the reservation quantity is increasing in $\theta$ for $\underline{\theta} \leq \theta<\theta^{c}(w)$, and flat and equal to $K$ for $\theta \geq \theta^{c}(w)$.

When the supplier's production capacity is not always sufficient to meet the consumer demand, the buyer makes his plans on the possibility of purchasing from the spot. In certain cases, especially when capacity is low and production costs are low, the buyer types with high marginal willingness to pay for the supplier's product over that of other suppliers' (i.e., types with high $\theta$ ) prefer to purchase the entire capacity of the supplier. This means that when the buyer type spectrum $[\underline{\theta}, \bar{\theta}]$ is sufficiently wide, there will be "mixed menus", in which at the lower end of the $\theta$ spectrum, each buyer type will have a different quantity to purchase, while at the higher end a group of buyer types will be bunched up, all buying the entire capacity, $K$, of the supplier. Low production costs also have implications on the contract and production plans. When $\beta<E[s](1-\phi) /\left(1+r_{S}\right)$, the supplier always produces up to capacity, $K$, since even if the buyer does not purchase all units produced, the supplier makes positive expected profits by selling $K-q^{*}(w, \theta)$ units directly to the spot market.

From Propositions 2 and 3 we can obtain the following corollary:

Corollary 1 If $r_{S}$ increases or there is an increase in $s$ in the sense of first-order stochastic dominance of distribution, the contract structure is more likely to be fully flat-price than mixed-menu, and more likely to be mixed-menu than fully volume dependent. Higher $K, r_{B}, w, \beta$, favor a volume-dependent reservation pricing scheme in the same sense.

As an agreement between the buyer and the seller, the contract reflects the incentives, preferences, and outside options of both the buyer and the supplier. Although the supplier determines the pricing of the goods she offers, she also needs to consider the buyer's incentives. As Corollary 1 indicates, a higher seller discount rate and a lower buyer discount rate move the pricing regime from a volume-dependent scheme to a flat price. Given $r_{S}>r_{B}$, i.e., the seller has a higher discount rate than that of the buyer, she has a higher valuation of payments made in the first period than in the second. Therefore, a higher $r_{S}$ makes it easier for the seller to commit to a large bundle up front. On the other hand, a higher $r_{B}$ reduces the buyer's willingness for commitment; the degree that the buyer wants to reduce the number of options he 
purchases also depends on his type, $\theta$, and consequently, a volume-dependent scheme becomes optimal for the supplier.

We can also see from Corollary 1 that higher production capacity moves contracting towards volume dependency rather than flat price. When the supplier has low capacity, an agreement in which the buyer reserves the entire capacity at a fixed price is more attractive for the parties since the buyer, regardless of his type, is likely to use a large part of the supplier's production. On the other hand, when the supplier's capacity increases, lower $\theta$ type buyers would unlikely be highly willing to buy all the capacity, and hence the seller would have to significantly reduce her price in order to sell to all types. Instead, she chooses to differentiate the intended quantities for lower and higher $\theta$ type buyers by volume-dependent pricing, and as a result, in the contract outcome at least some of the different buyer types purchase different quantities and pay accordingly. A higher exercise price $(w)$ can also switch the pricing regime from a flat-price scheme to a mixed-menu or volume-dependent scheme. This is because of a fundamental trade-off that the supplier faces when pricing the options contracts: the seller needs to strike a balance between receiving payments today in the form of reservation fees $(R(q))$, and receiving payments in the future in the form of exercise fees $(w)$. These two factors (imperfectly) substitute for each other in determining the pricing schedule for the contracts. When $w$ is large, the substantial part of the revenue for the supplier is in the second period. Given that the supplier has a higher discount rate than the buyer, she is less willing to commit to large quantities and hence switches to a sliding scale of commitment volume. A similar effect occurs when production cost $(\beta)$ is large, a high production cost reduces the supplier's incentive to commit to large quantities and shifts the pricing regime to a volume-dependent scheme as stated. Finally, when spot price is higher, i.e., if $s$ increases in the sense of first order stochastic dominance, it is more desirable for both the buyer and the seller to commit to a larger amount, and hence the seller is more likely to offer her entire capacity to the buyer.

The nature of volume dependency of the reservation price on the quantity, as reflected in the shape of $R(q)$ (e.g., convex or concave) will be discussed in the next section.

\subsection{Volume Dependency}

An important issue about the structure of the contract offer is the nature of volume dependency in the price schedule. A very common form of volume dependent pricing employed in practice is volume discounts; i.e., a reduction in average pricing with higher purchases. Volume discounts imply a concave total price curve as a function of quantity purchased. The opposite of volume discounts is volume premia; i.e., increasing average cost with quantity purchased. This is a reverse form of volume incentive, which can be viewed as extra incentives given to the seller by the buyer to commit to a high level of production. Conversely, it can be the case that the seller takes advantage of the premium the buyer puts on the seller's product by charging higher average prices to more dependent types of buyers (higher $\theta$ types). Contrary to volume discounts, volume premia imply convex total price curves. Given the complexity of the transaction structure at $t=2$ 
with options commitments and the resulting complexity of the two-sided determination of options contract terms, it is an open question whether either or both types of volume-dependent pricing structures can emerge in the optimal contracts. The following proposition states the conditions under which the optimal contract offers volume discounts and/or volume premia to the buyer at a given part of the reservation price curve.

Proposition 4 Suppose, for a given $w \geq 0$, the optimal contract is volume dependent.

(i) If $w \leq \underline{s}(1-\phi)$, then $R(q)$ is globally concave in q; i.e., the optimal contract offers quantity discounts for the entire reservation price curve, $R(q)$.

(ii) Suppose $w=\underline{s}+\tilde{\theta}$, where $\tilde{\theta} \in[\underline{\theta}, \bar{\theta}]$. Then $R(q)$ is locally concave at $q(\theta)$ if

$$
\rho \geq \frac{E\left[\left(\underline{s}+\tilde{\theta}-s(1-\phi)^{+}\right]\right.}{g_{\theta}(\theta)-\frac{d g_{\theta}(\theta)}{d \theta}(E[s]-\underline{s}+\theta-\tilde{\theta})+E\left[(s(1-\phi)-\underline{s}-\tilde{\theta})^{+}\right]},
$$

and locally convex at $q(\theta)$ otherwise.

Part $(i)$ of Proposition 4 states that for low $w$ values, the optimal contract only takes the shape of volume discounts. An important implication of this is for the case of $w=0$; i.e., when the contract is a "sales" contract rather than an "option" contract. The following corollary states the result.

Corollary 2 When the contract terms are such that the supplier is selling the intermediate good to the buyer (i.e., $w=0)$, volume-dependent pricing, if employed by the seller, always implies volume discounts.

This result is consistent with the widespread use of volume discount schemes for procurement sales contracts in most every industry and sector from food to chemicals, energy, and electronics (see, e.g., Wilson 1993 and Katz et al. 1994). Compared to options contracts, sales contracts are relatively simpler in that they do not offer flexibility to the buyer. The buyer commits to receiving all units at the time of the delivery and pays an upfront price for it. Corollary 2 states that as the level of such fixed commitments increases with the quantity sold, the seller finds it more profitable to offer incentives to the buyer to purchase more. These incentives strengthen the buyer's willingness to commit to larger quantities, increasing the supplier's profits.

On the other hand, option contracts are more complex agreements in terms of the possibilities they imply on the behavior of the involved parties. As a consequence, their volume-dependent pricing schedule is also more complicated. As part (ii) of Proposition 4 indicates, volume discounts can exist in the optimal contract for at least part of the offer curve for large option exercise price values as well. However, and remarkably, part (ii) of Proposition 4 also states that, unlike the reservation price curves for sales contracts, optimal option reservation price curves can exhibit volume premia instead of volume discounts. Further, it is possible that the same contract offer can involve volume discounts and volume premia at different 
parts of the pricing curve. So when and at what ranges do the optimal option contracts show concave and convex pricing characteristics? The following corollary sheds some light on this question.

Corollary 3 Suppose $w=\underline{s}+\tilde{\theta}$, where $\tilde{\theta} \in[\underline{\theta}, \bar{\theta}]$, and the optimal contract is volume dependent. Given $d^{2} g_{\theta}(\theta) / d \theta^{2} \geq 0$, (i) if $E\left[(w-s(1-p))^{+}\right] /\left(E[s]+\bar{\theta}-w+E\left[(s(1-\phi)-w)^{+}\right]\right)<\rho<\bar{\rho}$, where $\bar{\rho}$ is as defined in Proposition 2, then optimal contract has quantity discounts for all $q \geq q^{*}(w, \tilde{\theta})$; and (ii) if $0<\rho<E\left[(w-s(1-p))^{+}\right] /\left(g_{\theta}(\tilde{\theta})-\left(d g_{\theta}(\tilde{\theta}) / d \theta\right)(E[s]-\underline{s})+E\left[(s(1-\phi)-w)^{+}\right]\right)$, then the optimal contract has quantity premia for all $q \geq q^{*}(w, \tilde{\theta})$.

Corollary 3 states that both quantity discounts and quantity premia can easily be observed in large segments of the pricing curve. The condition $d^{2} g_{\theta}(\theta) / d \theta^{2} \geq 0$ is a relatively weak condition, satisfied by many common distributions such as exponential and uniform. Given these two conditions are satisfied, the high end of the pricing curve will demonstrate either volume discounts or volume penalties in its entirety. The former occurs for relatively high levels of supplier discount rates, and the latter is optimal for relatively low levels of it. To see an example for this corollary, consider a case with $\theta$ and $s$ distributions are uniform with supports $[2,3]$, and $[2,4]$ respectively, and $w=\underline{s}+\underline{\theta}=4$. For uniform $\theta$ distribution, the two bounds given in Corollary 3 are equal, because $g_{\theta}(\theta)=\bar{\theta}-\theta$, and hence $d g_{\theta} / d \theta=-1$. That is, for this example, there is a critical $\rho$ value $((1+3 \phi) / 2)$, above which the entire $R(q)$ curve is concave, and below which the entire curve is convex. For a range of $\phi$ values, from 0.1 to 0.5 , for instance, the critical $\rho$ is on the range 0.65 to 1.25 .

Another interesting issue is the effect of the exercise price, $w$, on the expected contracted quantity, which is presented in the following proposition.

\section{Proposition 5}

(i) If $r_{S} \geq r_{B}$, expected contracted quantity, $E\left[q^{*}(w, \theta)\right]$, decreases in the exercise price $w$.

(ii) If $r_{S}<r_{B}, E\left[q^{*}(w, \theta)\right]$ increases in $w$ for $w<\underline{s}+\underline{\theta}$, and can be increasing or decreasing in $w$ for $w \geq \underline{s}+\underline{\theta}$.

Part $(i)$ of Proposition 5 states that given the seller has a higher discount rate than the buyer, when the exercise price increases, she does not decrease the reservation price substantially enough to generate increased buyer demand for the options. As a consequence, the expected total number of option contracts decreases with increased exercise price, as can also be seen in panel $(a)$ of Figure 1. However, when the seller has a lower discount rate than the buyer, for low $w$ values, she may find it optimal to decrease the reservation price significantly in the optimal contracts to increase the quantity contracted. Even then, beyond a certain threshold value, as $w$ increases, the value of the options diminish sharply, making it not worthwhile for the seller to reduce the reservation price to keep the contracted quantity high, as also stated in part (ii) of Proposition 5. Consequently, the number of options sold can be maximized at an 

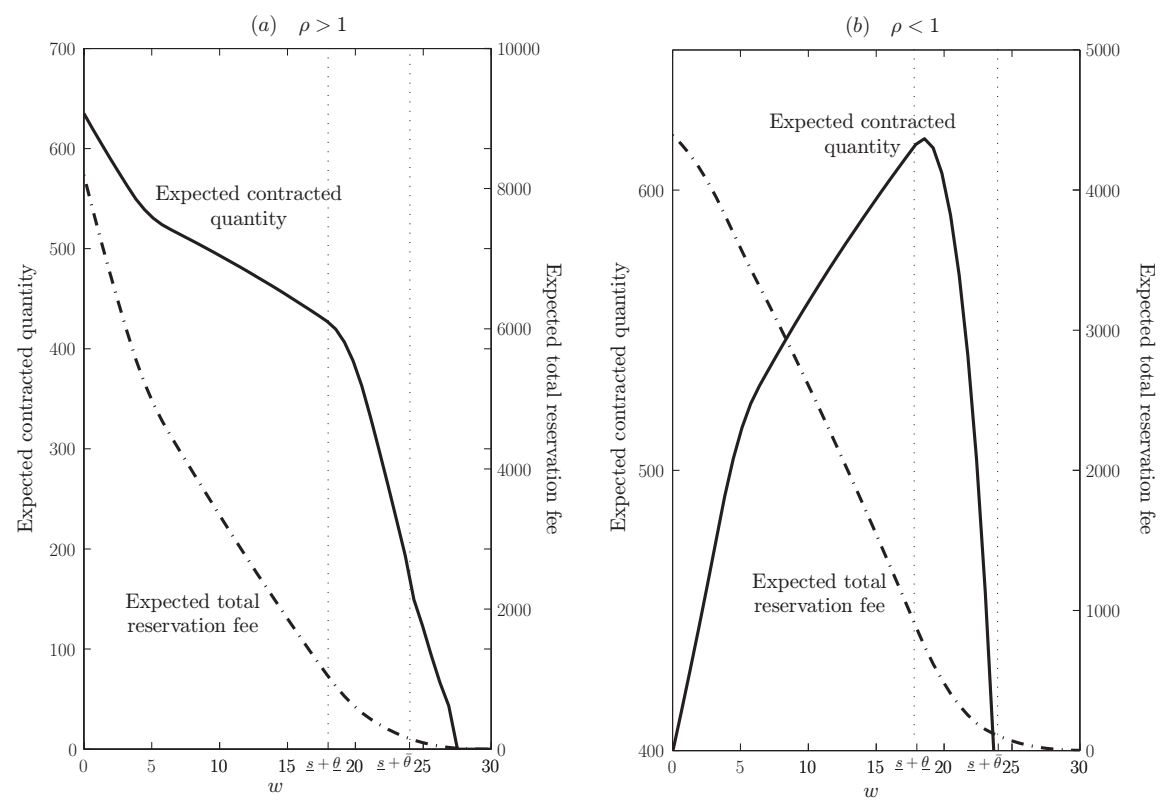

Figure 1: The effect of the exercise price $(w)$ on the expected contracted quantity $\left(E\left[q^{*}(w, \theta)\right]\right)$, and the expected reservation fee collected by the supplier $\left(R\left(q^{*}(w, \theta), w\right)\right)$. For panel (a) $\rho=1.36$, and for panel (b) $\rho=0.73$. For both panels, $s, \theta$ and $D$ have truncated normal distributions on $[10,18],[8,14]$, and $[0,1200]$, means 14, 11 and 600, and standard deviations 5, 4 and 100, respectively. The remaining parameters are $p=40, \beta=8$, and $\phi=0.65$.

intermediate $w$ level, as can also be seen in panel $(b)$ of Figure 1. However, as can further be seen in the figure, the reservation price decreases with increased exercise price, even though the number of contracts signed increases sharply, as demonstrated in panel $(b)$. We will further use the results of Proposition 5 when we explore the effects of parameters on optimal contract design in Section 6.1.

\subsection{Determination of the Optimal Exercise Price and Contract Structure}

Having found the optimal contract structure for a fixed exercise price $w$, we can now explore the determination of optimal exercise price. Our analysis will parallel the general outline of the exercise price optimization given in Section 4. The major and consequential difference is the randomness of the buyer type, $\theta$. By using (11), the supplier's global optimization problem is now

$$
\max _{w \geq 0} \pi_{S}(w) \equiv \max _{w \geq 0} \int_{\underline{\theta}}^{\bar{\theta}}\left[R\left(q^{*}(w, \theta), w\right)+V\left(q^{*}(w, \theta), w, \theta\right)\right] d F_{\theta}(\theta)
$$

We again start with the case where $K \geq \bar{D}$.

Proposition 6 Suppose $K \geq \bar{D}$, and $\rho<\bar{\rho}$, where $\bar{\rho}$ is as defined in Proposition 2.

(i) If $r_{S}>r_{B}, w^{*}=0$. That is, selling the intermediate good is optimal for the supplier, rather than 
(a) $\rho>1$

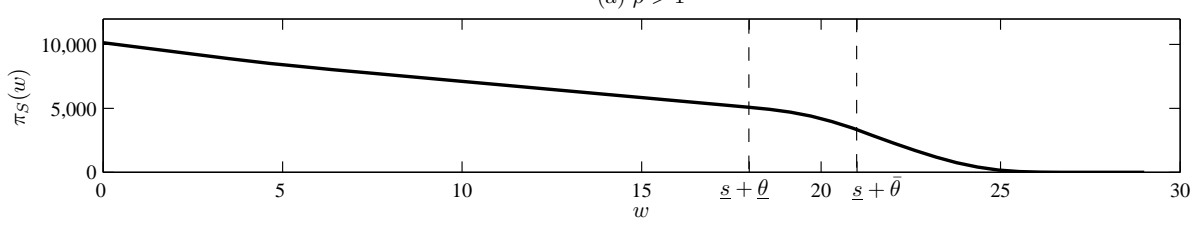

(b) $\rho=1$
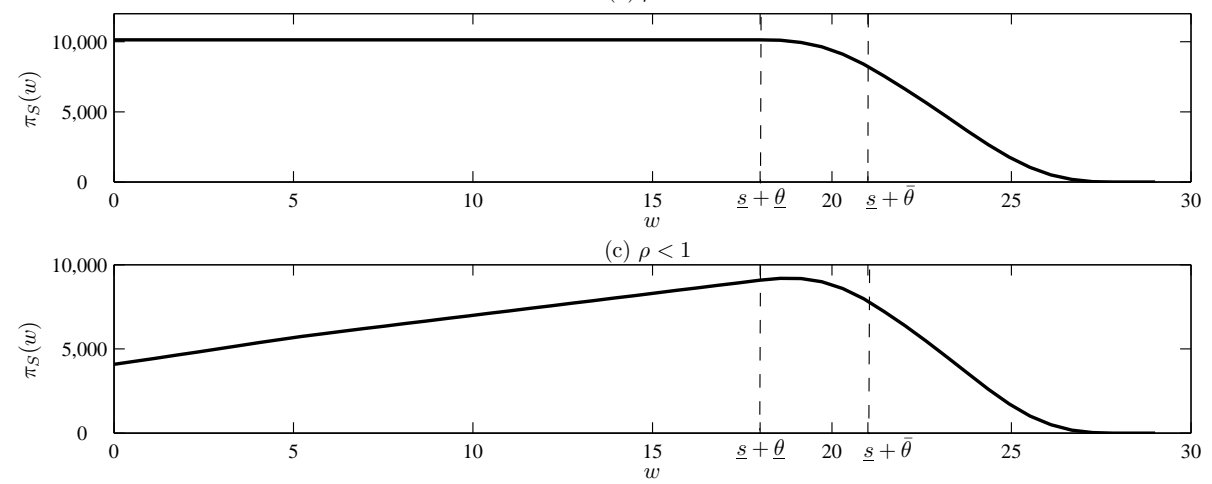

Figure 2: The supplier's expected profit $\left(\pi_{S}\right)$ curves as a function of the exercise price, $w$, for the three main regions stated in Proposition 6. In panel (a), $\rho=1.5$, in panel (b) $\rho=1$, and in panel (c) $\rho=2 / 3$. In all panels, $s$ and $\theta$ have truncated normal distributions on $[10,18]$ and $[8,11]$, means 14 and 9.5 , and standard deviations 5 and 1.5, respectively. Demand distribution is increasing triangular with support $[0,1200]$. The remaining parameters are $p=30, \beta=8$, and $\phi=0.65$.

offering options to the buyer.

(ii) If $r_{S}=r_{B}$, the seller's profit is maximized by setting any exercise price $w^{*}$, where $0 \leq w^{*} \leq \underline{s}+\underline{\theta}$.

(iii) If $r_{S}<r_{B}, \underline{s}+\underline{\theta}<w^{*} \leq \bar{s}+\bar{\theta}$, i.e., the seller prefers offering options to selling the intermediate good.

Figure 2 depicts the seller's expected profit as a function of the exercise price $w$. As can be seen from panels (a) and (b) of this figure, similar to the case with fixed $\theta$, for $r_{S}>r_{B}$, optimal contracts are sales contracts with $w^{*}=0$; and for $r_{S}=r_{B}$, there is a continuum of optimal exercise prices, in this case on $w \in[0, \underline{s}+\underline{\theta}]$. For $r_{B}>r_{S}$, however, the optimal exercise price is increasing in this case all the way up to and beyond $\underline{s}+\underline{\theta}$, as can be seen in panel (c) of Figure 2, and is optimized for a $w^{*} \in(\underline{s}+\underline{\theta}, \underline{s}+\bar{\theta})$. This means that the supplier offers true option contracts to the buyer with a non-zero probability that the buyer will not exercise the options he buys. This is because the seller is facing a trade-off between increasing the exercise price and increasing the quantity of the options sold, and the increased exercise price pays off by increasing the expected profits while keeping the number of options sold high.

Combining the results of Proposition 6 with those of Propositions 2 and 4, we can deduce the characterization of full optimal contract structure for $K>\bar{D}$ and $\beta>E[s](1-\phi)\left(1+r_{S}\right)^{-1}$, which is summarized in panel (a) of Figure 3. Recall Proposition 2 stated that when the seller has a higher discount rate than the buyer and production costs are sufficiently low, the optimal contract is a flat-price contract. Thus, 
by Proposition 6, optimal contracts in this region will be flat-price sales contracts. When $r_{S}>r_{B}$, if the production costs are beyond the critical threshold, the optimal contracts are volume-dependent. Further, by Proposition 4 (or more specifically Corollary 2), since these contracts are sales contracts, they will demonstrate volume discounts as also indicated in panel (a) of Figure 3. On the other hand, if the buyer has a higher discount rate than the seller (i.e., $\rho<1$ ), then the optimal contract is a volume-dependent option contract. Further, since by Proposition 6 the optimal exercise price is between $\underline{s}+\underline{\theta}$ and $\underline{s}+\bar{\theta}$, by part (ii) of Proposition 4 the reservation price curve can have concave and convex parts; i.e., the optimal contract offer can have volume discounts on the options as well as volume premia or both.

Parallel to our analysis in Section 5, we can also expand the results of Proposition 6 to low capacity case.

Proposition 7 Suppose $K<\bar{D}$, and $\rho<\bar{\rho}$, where $\bar{\rho}$ is as defined in Proposition 2.

(i) If $r_{B} \leq r_{S}$, then $w^{*}=0$. That is, sales contracts are optimal for the seller rather than options.

(ii) If $r_{B}>r_{S}$, then there exists $w^{*} \in[0, \underline{s}+\bar{\theta}]$, that optimizes seller's profit. Moreover,

(a) If $E[s]+\bar{\theta}-g_{\theta}(\bar{\theta})>\beta\left(1+r_{S}\right)$, then there exists a cutoff value $\gamma, \underline{K}>0$, such that if $r_{B} \leq r_{S}+\gamma$, and $K<\underline{K}$, then $w^{*}=0$; i.e., sales contracts are optimal.

(b) There exists a $\bar{K}>0$ such that, if $K>\bar{K}$ then $w^{*} \in(\underline{s}+\underline{\theta}, \bar{s}+\bar{\theta}]$. That is, the seller prefers offering options to selling the intermediate good.

Panel (b) of Figure 3 demonstrates the contract structure in relation to capacity and the relative discount rates of the buyer and the seller, captured by $\rho=\left(1+r_{S}\right) /\left(1+r_{B}\right)$. The first thing to notice is that, unlike the case with $K>\bar{D}$, sales contracts can be optimal even when the buyer's discount rate is higher than that of the seller's, provided that the seller's capacity is sufficiently low. This is because, given the seller's capacity is low enough, there is a strong chance that the buyer will use most or all of this capacity at the time demand is realized. Then, provided that the buyer's discount rate is not too high compared to that of the seller, the seller may be better off offering the buyer to pay up front for the entire capacity at a slightly lower price instead of offering a higher exercise price, volume-dependent contract. As production capacity, $K$, increases, or the buyer's discount rate becomes higher compared to the seller's (i.e., $\rho$ decreases), the contract structure moves from flat price to mixed-menu to full volume discount, as was noted by Corollary 1. Note that, given the buyer's discount rate is higher than the seller's, once the capacity is large enough the contract is always fully volume dependent and involves a positive exercise price; i.e., in this case the contract is always a volume-dependent options contract. 

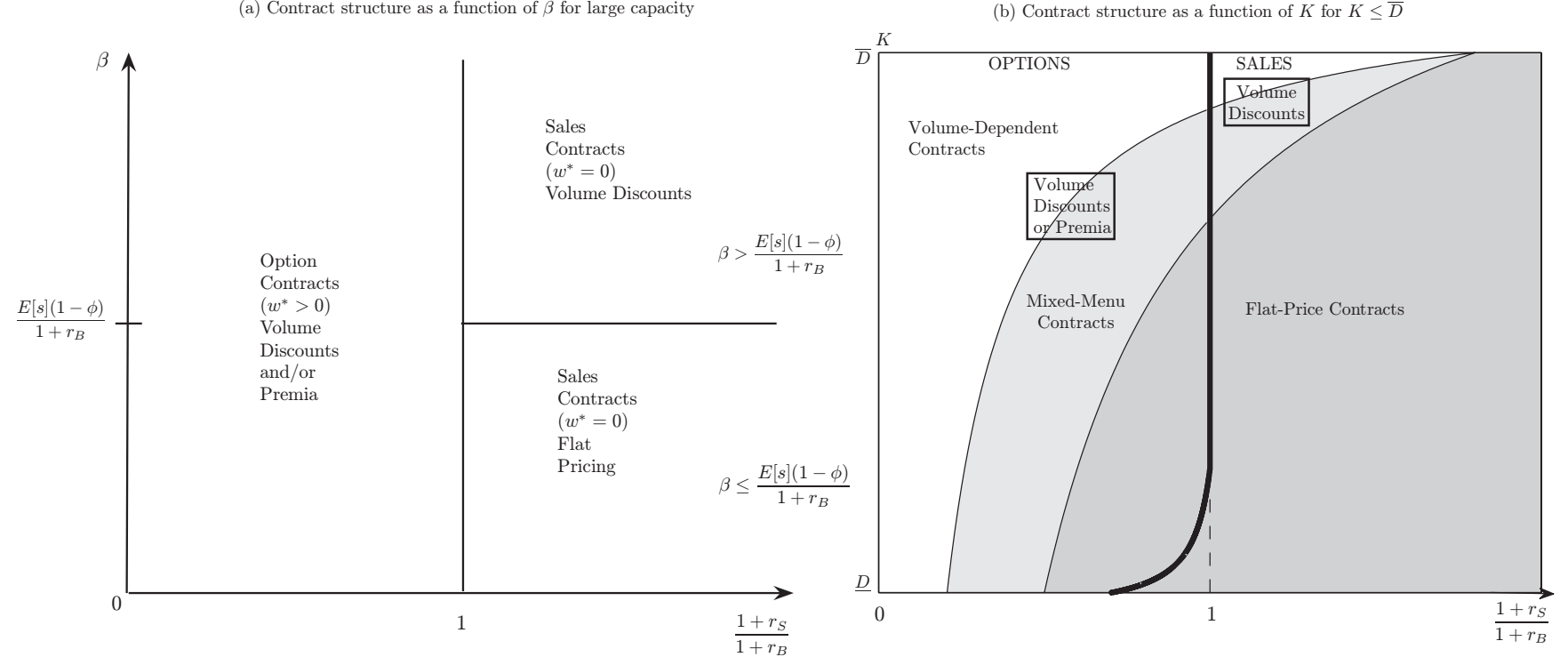

Figure 3: Contract structure characterization. Panel (a) illustrates the partition of the parameter space according to the characterization of contract structures for $K \geq \bar{D}$. The horizontal axis specifies $\rho$ (i.e., $\left.\left(1+r_{S}\right) /\left(1+r_{B}\right)\right)$, and the vertical axis specifies $\beta$. Panel (b) illustrates the partition under limited production capacity, $K$, with the vertical axis specifying $K$. The solid line separates sales contracts from options. The darkest shaded region indicates flat-price contracts, the medium shaded one indicates mixedmenu contracts, and the unshaded region indicates fully volume-dependent contracts.

\section{Contract Characteristics}

\subsection{The Effects of Parameters on Contract Design}

We next examine the effects of buyer, seller, and market characteristics (such as production costs, spot price distribution, bid-ask spread in the spot market, and information asymmetry between the buyer and the seller about buyer's production flexibility) on the optimal contract design, including exercise price, reservation price, and expected contracted quantity. In the remainder of the paper, for expositional simplicity, for increases in expected value of a random variable while preserving the shape of the distribution $(\theta$ or $s)$, we will use the term rightward shift in the probability distribution; i.e., $f_{\theta}(\theta+x)$ in the new distribution equals $f_{\theta}(\theta)$ in the old distribution for all $\theta$ and a constant $x>0$. Similarly, when we talk about increased variance, we will refer to mean preserving spreads that strictly reduce the infimum of the support of the distribution. ${ }^{10}$ Also note that for simplicity, for the entire Section 6, we will focus on the case of $K \geq \bar{D}, \beta \geq E[s](1-\phi)\left(1+r_{S}\right)^{-1}$, and $\rho<\bar{\rho}$, where $\bar{\rho}$ is as defined in Proposition 2.

We start with the effects on exercise price. The following proposition states the result.

Proposition 8 If $r_{B}>r_{S}$, then there exists $\delta_{1}>0$ such that when $1-\delta_{1}<\rho<1$, the optimal exercise

\footnotetext{
${ }^{10}$ The definition applies as long as the shift reduces the infimum of the distribution's support whether or not it increases the supremum of the support.
} 
price, $w^{*}$, increases with a rightward shift in the distribution of $\theta$ or $s$. It decreases with $\rho$, or if $\operatorname{Var}[s]$ or $\operatorname{Var}[\theta]$ increase with a mean preserving spread that reduces the infimum of the support of the distribution.

As the spot price or the buyer's sourcing inflexibility (measured by $\theta$ ) increases, buyer's alternative sources of procurement at $t=2$ become less attractive. Hence, the seller can charge a higher exercise price for the options. On the other hand, when $\rho$ increases, the buyer becomes values future payoffs less compared to the seller. Consequently, the seller can gain by offering contracts that shift the fee collection to $t=1$ by decreasing the exercise price. If the spot price distribution spreads out, then the probability that the buyer exercises his options decreases since it is more likely that the spot price will be lower than the option's exercise price. This decrease forces the supplier to reduce the exercise price to make the options more attractive to the buyer. Finally, when the uncertainty on the buyer's type increases, the probability that the buyer exercises his options decreases, inducing the supplier to reduce the exercise price of the options in the optimal contracts.

Another important measure is the expected quantity contracted between the buyer and the seller, i.e., $E_{\theta}\left[q^{*}(w, \theta)\right]$. The next proposition summarizes the effects of industry and market parameters on expected contracted quantity.

\section{Proposition 9}

(i) If $r_{B}<r_{S}$, expected contracted quantity, $E_{\theta}\left[q^{*}(w, \theta)\right]$, increases with an increase in $\rho$, or with a rightward shift in the distribution of $\theta$ or $s$; and it decreases with $\phi$ and $\beta$.

(ii) If $r_{B} \geq r_{S}$, then there exists $\delta_{2}>0$ such that when $1-\delta_{2}<\rho<1$, expected contracted quantity, $E_{\theta}\left[q^{*}(w, \theta)\right]$, increases with an increase in $\rho$, or with a rightward shift in the distribution of $\theta$ or $s$. It decreases with $\phi, \beta$, or if $\operatorname{Var}[s]$ increases with a mean preserving spread that reduces the infimum of the support of the distribution.

As mentioned above, increased spot price and increased buyer inflexibility increases the attractiveness of the options to the buyer. This increases the number of contracts purchased for both cases, $w^{*}=0$ and $w^{*}>0$. An increased bid-ask spread reduces the amount the buyer can recoup for the intermediate goods he cannot use to satisfy consumer demand, making the options less attractive. As a result, the number of contracts he purchases decreases. An increase in spot price variance increases the probability that the buyer can supplement his procurement from the spot market at time $t=2$. Consequently, when the seller is offering options, i.e., when $w^{*}>0$, the options become less crucial for him and the expected contracted quantity decreases.

Finally, we examine how the expected reservation fee changes with industry and market characteristics. The following proposition states the result. 


\section{Proposition 10}

(i) If $r_{B}<r_{S}$, expected reservation fee paid, $E_{\theta}\left[R\left(q^{*}\left(w^{*}, \theta\right), w^{*}\right)\right]$, increases with $\rho$, or with a rightward shift in the distribution of $s$ or $\theta$; and it decreases with $\phi$ and $\beta$.

(ii) If $r_{B} \geq r_{S}$, then there exists $\delta_{3}>0$ such that when $1-\delta_{3}<\rho<1$, expected reservation fee paid, $E_{\theta}\left[R\left(q^{*}\left(w^{*}, \theta\right), w^{*}\right)\right]$, increases with an increase in $\delta$, or with a rightward shift in the distribution of $\theta$ or $s$, or when $\operatorname{Var}[s]$ increases with a mean preserving spread that reduces the infimum of the support of the distribution. Further, when $1-\delta_{3}<\rho<1$, expected reservation fee paid, $E_{\theta}\left[R\left(q^{*}\left(w^{*}, \theta\right), w^{*}\right)\right]$, decreases with $\phi$ and $\beta$.

As before, an increase in the expected spot price or the buyer's expected inflexibility makes the options more valuable to the buyer. Consequently, the supplier increases the reservation price in the optimal contracts for both cases with $w^{*}=0$ and $w^{*}>0$. The effects of increase in discount rate differences and the spot price variance filter through the supplier's trade-off between the reservation and exercise prices of the options. As the supplier's discount rate becomes higher compared to the buyer, she prefers to shift her revenues to earlier rather than later. Consequently, she decreases the exercise price as stated in Proposition 8 and increases the reservation price. Further, an increase in spot price variance decreases the probability of the buyer exercising the options for any given fixed $w$. In response, the supplier finds it optimal to decrease the exercise price to increase the exercise probability. Part (ii) of Proposition 10 states that in the optimal contracts, the supplier is able to recuperate some of her losses from this decrease in exercise price by increasing the reservation fees. Lastly, an increase in the spot market bid-ask spread has a strong effect in reducing the value of the options. So much so that, even though the seller decreases the exercise price in the contract offer, she still has to reduce the reservation fees in order to maximize her profits.

\subsection{Uncertainty and Efficiency}

In this section, we provide a numerical analysis of the determinants of contract characteristics and supply chain efficiency. Specifically, we focus on the effects of distributions, i.e., mean and variance, of the buyer's sourcing flexibility and the spot price, which are two important factors in determining contract characteristics.

\subsubsection{The First-Best Solution}

In order to obtain a yardstick for measuring efficiency, we first calculate the first-best supply chain profit. The supply chain first-best is achieved under the assumption of an integrated channel with centralized decision making and no information asymmetry. Consider the integrated channel where the downstream unit has type $\theta$, i.e., purchasing the good from the outside supplier results in a value loss of $\theta$, and the 
production level $y(\theta)$ at $t=1$ is determined centrally. The corresponding supply chain profit level is then

$$
\pi_{S C}(y, \theta)=-\beta y+\frac{1}{1+r_{S C}}\left(p E[D]+E[s](1-\phi) E_{D}\left[(y-D)^{+}\right]-(E[s]+\theta) E_{D}\left[(D-y)^{+}\right]\right)
$$

where $r_{S C}$ is the discount rate for the integrated supply chain. With this set up, the following proposition presents the coordinated supply chain's first-best solution.

Proposition 11 The expected highest attainable profit level for the centralized system is

$$
\pi_{F B}=\frac{1}{1+r_{S C}}\left((p-E[s]-E[\theta]) E[D]+\int_{\underline{\theta}}^{\bar{\theta}}(\phi E[s]+\theta) \int_{0}^{y^{*}(\theta)} x d F_{D}(x) d F_{\theta}(\theta)\right),
$$

where $y^{*}(\theta)$ denotes the optimal production level of the centralized system with a type $\theta$ downstream unit and is given by

$$
y^{*}(\theta)=\bar{F}_{D}^{-1}\left(\frac{\left(1+r_{S}\right) \beta-E[s](1-\phi)}{\phi E[s]+\theta}\right) .
$$

In general, firm size is inversely related to a firm's discount factor or cost of capital (see, e.g., AshbaughSkaife and LaFond 2006, Berk and DeMarzo 2007, Hail and Leuz 2009 among others). Smaller firms normally borrow at higher interest rates than larger firms. One main reason for that is smaller firms, in general, are riskier than the larger ones (e.g., higher default rate). Therefore, since the integrated supply chain combines the supplier and the buyer, the discount rate for the centralized channel $r_{S C}$ should be considered as lower than both $r_{S}$ and $r_{B}$; i.e., $0 \leq r_{S C} \leq \min \left\{r_{S}, r_{B}\right\}$. We consider this range in our numerical analysis for the integrated channel's discount rate.

Utilizing the first-best solution as described in Proposition 11, we can now calculate the percentage efficiency of the optimal procurement contracts and the division of the surplus between the supplier and the buyer. Table 1 provides the contract characteristics as the spot price and buyer flexibility distributions and the relative discount rates of the seller and the buyer $(\rho)$ vary. Table 2 provides the corresponding efficiency measures. The tables present three sourcing-flexibility distribution cases to explore the effects of shifts in the expected value and variance: base (or "standard") case $(\theta \sim U[20,24]$, denoted by "ST"), a variancepreserving case $(\theta \sim U[28,32]$, denoted by "VP"), and a mean-preserving case $(\theta \sim U[15,29]$, denoted by "MP"). In addition to measuring supply chain efficiency with respect to the first-best $\left(\pi_{S C} / \pi_{F B}\right)$ and the supplier's share of the supply chain surplus $\left(\pi_{S} / \pi_{S C}\right)$, we also measure the percentage gain from employing an options contract compared to employing a traditional sales contract (i.e., with $w^{*}=0$ ) for the supplier $\left(\Gamma_{S}=\left(\pi_{S}\left(w^{*}\right)-\pi_{S}(0)\right) / \pi_{S}(0)\right)$ and the supply chain $\left(\Gamma_{S C}=\left(\pi_{S C}\left(w^{*}\right)-\pi_{S C}(0)\right) / \pi_{S C}(0)\right)$ as a whole.

\subsubsection{The effect of the distribution of the buyer's sourcing flexibility}

There are two underlying effects of decreased buyer flexibility (increased $\theta$ ) on supply chain efficiency and surplus distribution. First, reduced buyer flexibility reduces the ability of the supply chain as a whole to respond quickly to spot market and demand realizations, and hence decreases the overall efficiency of the 

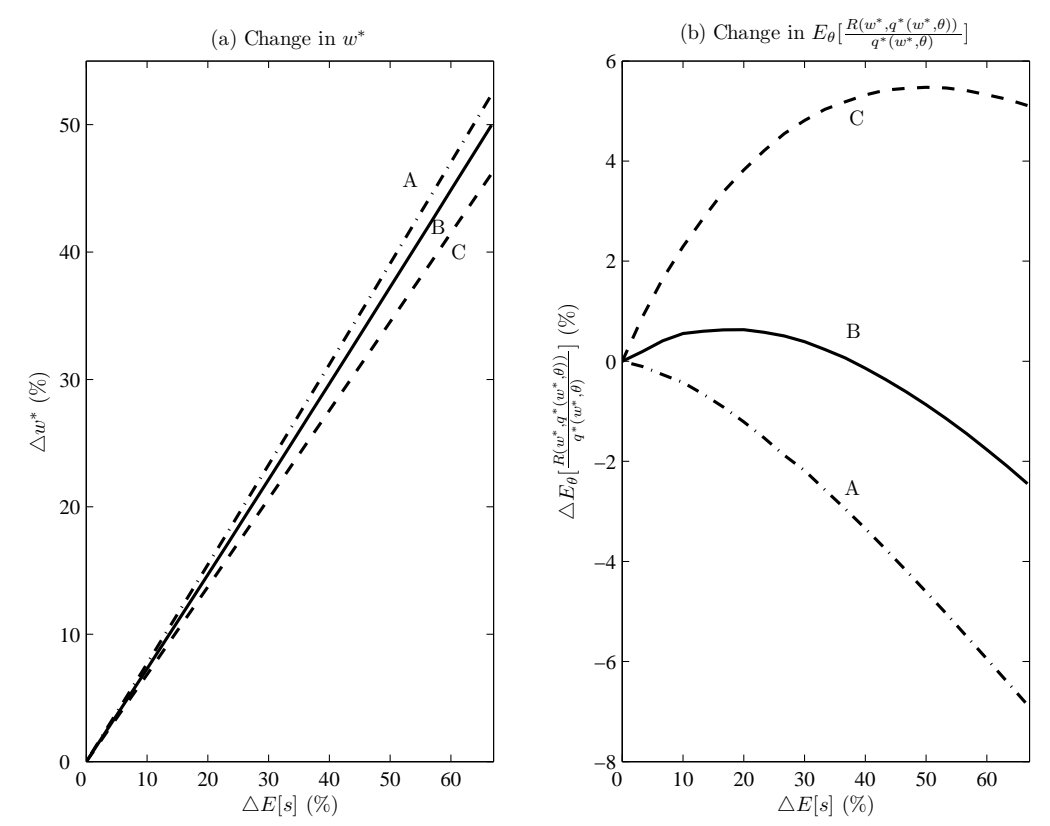

Figure 4: Percentage change in the optimal exercise price $w^{*}$ (panel (a)), and average reservation price $E_{\theta}\left[R\left(q^{*}\left(w^{*}, \theta\right), w^{*}\right) / q^{*}\left(w^{*}, \theta\right)\right]$ (panel (b)) with increase in expected spot price. For case $A, \rho=0.9$, for cases $B$ and $C, \rho=0.8$. The spot price $s$ has a truncated normal distribution with standard deviation 40 and support $[E[s]-9.5, E[s]+9.5]$ for cases $A$ and $B$, and standard deviation 3 and support $[E[s]-4, E[s]+4]$ for case $C$. For all cases, the demand has truncated normal distribution with mean 600, standard deviation 400 with support $[0,1200]$; and $\theta$ is $U[15,29]$. The remaining parameters are $p=100, \phi=0.75, \beta=13$, and the initial mean spot price is 30 .

supply chain. Second, increased $\theta$ increases the dependence of the buyer to the supplier. Increased buyer dependence shifts surplus from buyer to the seller, but also increases the contracted quantity at $t=1$, moving it closer to the first-best quantity, increasing supply chain efficiency. An increase in $\theta$ has inverse effects for the efficiency of the options and sales contracts. As Table 1 demonstrates, for $r_{S}<r_{B}$, increased $\theta$ induces the supplier to shift her fee collection from reservation $\left(E_{\theta}\left[R\left(q^{*}(\theta)\right) / q^{*}(\theta)\right]\right)$ to exercise $\left(w^{*}\right)$. This is because, for this range, the supplier has a lower discount rate than the buyer and takes advantage of the buyer's increased additional cost of outside procurement, pricing the contracts to delay payments. As a result, the contracted quantity increases significantly, and the negative effect of decreased production flexibility is mitigated by reduced exercise price, $w^{*}$, resulting in increased supply chain efficiency as can be observed in Table 2. For $r_{S} \geq r_{B}$ on the other hand, a decrease in buyer's sourcing flexibility reduces supply chain efficiency, as $w^{*}=0$ in this region; i.e., sales contracts are optimal, and the supplier incorporates the increased additional cost of the buyer's outside procurement on the reservation fees, increasing her expected profits but decreasing the supply chain efficiency overall.

An increase in $\theta$ spread, i.e., an increase in $\operatorname{Var}[\theta]$, again has two separate effects. First, increased uncertainty on $\theta$ increases the information asymmetry between the buyer and the seller, thereby increasing the information rent the seller gives to the buyer. Second, an increase in the $\theta$ spread increases the 
probability of the buyer to rely on spot purchases when possessing options to the supplier's capacity. This is because, under option contracts, for any given spot price realization $s$, any increase in the probability of higher $\theta$ does not change whether the buyer exercises the options, since he exercises them at the fixed fee $w$ beyond a certain $\theta$ value. On the other hand, an increase in the probability of lower $\theta$ values would directly decrease the probability of the exercise of options and his procurement costs. As can be seen from Table 1 , both of these effects force the seller not only to decrease her average unit reservation price but also the exercise price for the options. Consequently, the buyer's surplus increases as Table 2 displays. However, increased information asymmetry between the seller and the buyer reduces the operating efficiency of the supply chain. Interestingly, the supplier's relative gains from employing the optimal options contract versus a traditional sales contract $\left(\Gamma_{S}\right)$ increases with increased variance of flexibility, as the buyer's willingnessto-pay for option contracts increases compared to committing to sales contracts. In contrast, the supply chain's benefit from employing option contracts decreases, because the total quantity contracted between the buyer and the seller decreases and moves away from the first-best contract quantity.

\subsubsection{The effect of the spot price distribution}

As the expected spot price increases, the buyer becomes more dependent on contracts with the supplier. As a consequence the supplier can increase the fees she charges. However, increased spot price also has an effect on the shape of the reservation price schedule $R(q)$. Specifically, by inequality (17) given in Proposition 4, an increase in $E[s]$ increases the concavity of the reservation schedule $R(q)$ at the higher end of the curve, which flattens the price schedule and reduces the expected unit price. The role of these two effects on contract characteristics can be observed in Table 1 as well as in Figure 4. As can be seen in Table 1, for $r_{S} \geq r_{B}$, an increase in the expected spot price is reflected as an increase in the average reservation fee. For $r_{S}<r_{B}$, the seller's response to a spot price increase involves increasing the exercise price, as panel (a) of Figure 4 illustrates. The average unit reservation price, on the other hand, can increase or decrease with an increase in spot price, as can be seen in panel (b). Specifically, the average unit reservation fee can increase with $E[s]$ when $r_{S}$ is substantially lower than $r_{B}$ (i.e., when $\rho$ is low) and at low $E[s]$ values (see cases $B$ and $C$ in the figure). However, as the inequality (17) points out, increased $E[s]$ tends to flatten the reservation fee schedule and eventually decrease average unit reservation price. The effect of an expected spot price increase on the relative efficiency of the supply chain depends on the relative magnitudes of $r_{B}$ and $r_{S}$. When $r_{S}>r_{B}$, the supply chain operates under sales contracts that are more rigid than the option contracts employed when $r_{S}<r_{B}$. With option contracts, an increase in spot price can cause an increase in supply chain efficiency, as can be seen in panel (a) of Figure 5, while with sales contracts, an increase in $E[s]$ reduces the supply chain surplus faster than it reduces the first-best, as demonstrated in panel (b). Thus, option contracts can serve as a "shock absorbent" for the whole supply chain against inclines in spot prices. This is also evident in the increase in the value of the options relative to the sales contracts for the supply chain $\left(\Gamma_{S C}\right)$ with increased expected spot price, as can be seen in Table 2 for cases with $r_{S}<r_{B}$. 


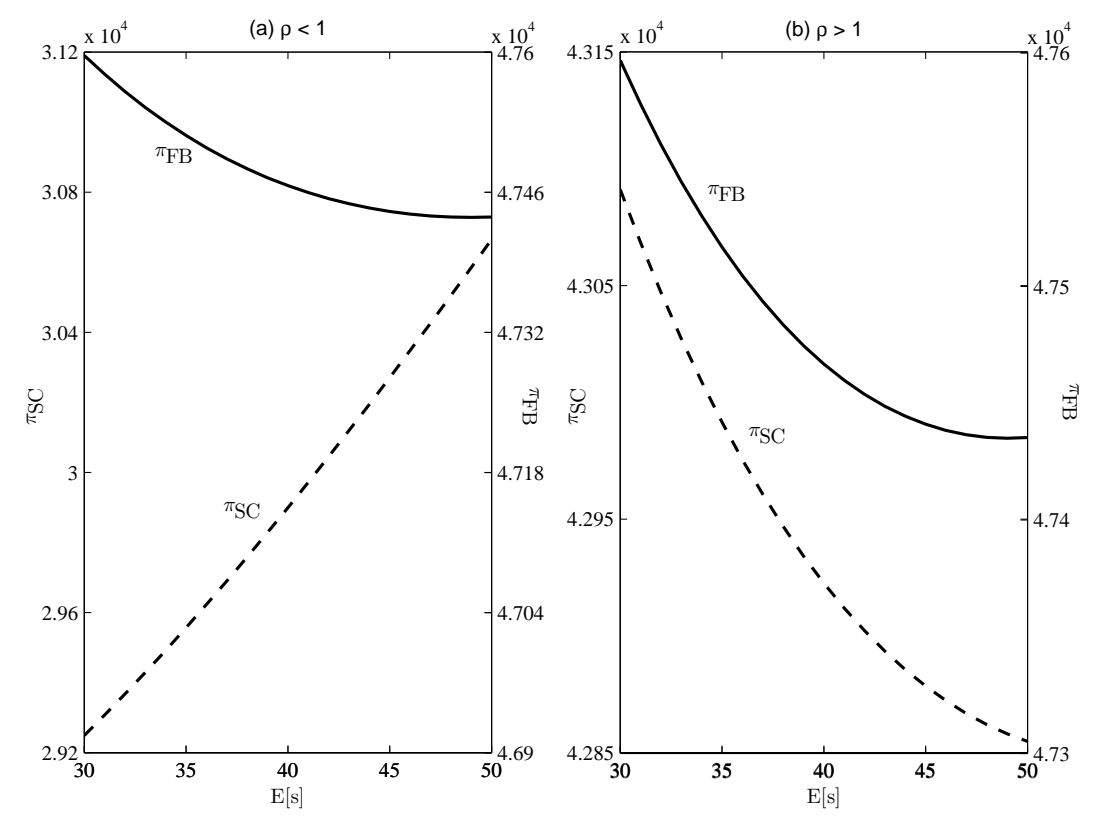

Figure 5: The contrast between the effect of increase in expected spot price on supply chain efficiency with option contracts (panel (a), $\rho=0.8$ ) and sale contracts (panel (b), $\rho=1.2$ ). For both panels, $s$ has truncated normal distribution with standard deviation 3 and support $[E[s]-4, E[s]+4] ; D$ has truncated normal distribution with mean 600 , standard deviation 400 with support $[0,1200]$; and $\theta$ is $U[20,24]$. The remaining parameters are $p=100, \phi=0.95$, and $\beta=13$.

Finally, when spot price variance increases, given the expected spot price stays constant, the relative surplus distribution with the expected sales contracts (which are optimal for the supplier for $r_{S} \geq r_{B}$ ) do not change as the contracts are rigid and their use does not depend on the realization of the spot price. However, for $r_{S}<r_{B}$, option contracts are optimal and the outcome is sensitive to increased spot variance. For option contracts, an increase in the spot price variance introduces an asymmetric effect similar to an increased variance of the buyer's sourcing flexibility as we discussed above. Specifically increased likelihood of lower spot price realizations, $s$, directly increases the buyer's expected profits, while beyond a certain point, the increased spread on the higher end has no effect on the outcome, since for large enough $s$, the buyer exercises the options at the fixed exercise price. Consequently, increased spot price variance benefits the buyer and makes the option contracts with the supplier less valuable. To counter this effect, in order to make the option contracts more attractive at lower spot price realizations, the supplier decreases the exercise price, but sharply increases the unit reservation price to compensate as can be seen from Table 1. However, due to the advantage increased spot variance provides to the buyer, the supplier's share of the surplus decreases, as Table 2 demonstrates. Further, the expected benefits of employing option contracts compared to sales contracts for the supplier and the supply chain also decrease. Supply chain efficiency also suffers from increased uncertainty in the spot market since such an increase in uncertainty substantially increases per-unit reservation fees and reduces the contracted quantities. 


\section{Discussion and Extensions}

In this section, we discuss our model's assumptions and limitations, as well as some related extension suggestions.

We assumed that the spot price distribution is not affected by the contracting between the buyer and the seller. This is a common assumption employed by most papers that study the subject in the literature (see, e.g., Peleg et al. 2002, Wu et al. 2002, as well as the references cited in Kleindorfer and $\mathrm{Wu}$ 2003). One of the main reasons for this is tractability. Making spot price dependent on contracting outcome significantly increases complexity as one has to model the entire microstructure of spot trading and incorporate it with the rest of the model, and that severely hurts the tractability of the model. ${ }^{11}$ In addition, in many cases, with a relatively small number of sellers, many markets may become nearly competitive or highly liquid (see, e.g., Tunca 2008, which explicitly models endogenous market liquidity). The specific number of sellers needed for this to happen changes depending on the structure of trading in the market for instance, with Bertrand style competition, even two sellers is sufficient to make the market competitive. Also it should be noted that for "spot trading" to take place, there does not need to be a specifically designed "spot market". Spot purchases, in fact can be any purchase that is made at or near the time of demand fulfilment. As such, given that there are multiple suppliers available, the market can be reasonably liquid.

Again, similar to most studies in the literature, we assumed there is no limit on the quantity of spot purchases. This assumption also helps with tractability of the model. Further, although there is clearly a finite amount of supply available in any market, in most cases many companies do not have large enough demand to exhaust the entire supply in the industry. Finally, the unlimited spot capacity is not a main driver of our major qualitative results. We believe if one added a finite capacity to the spot market, the main structure of our results would mostly be preserved. Nevertheless, it can be a worthwhile extension direction to study the effect of limited supply availability in the spot purchases. One effect this could have on contracting would be increased overall prices (reservation and/or exercise) for options, and perhaps an increase in the sales contracts instead of options because of the increased value of commitment for the buyer.

In our model, we have information asymmetry between the buyer and the supplier on the buyer's willingness to pay for the supplier's product over other suppliers' products $(\theta)$. In contrast, the discount rates for the buyer and the seller are known to the other party. As we have discussed in Section 3, buyers are often willing to pay a premium for the parts procured from their preferred supplier(s). This premium is idiosyncratic and private for each firm and for a given time point, and consequently, it is almost always private information. The buyer keeps this information to himself and would avoid revealing it to the supplier. Therefore, there is almost always an information asymmetry between the buyer and the seller about this premium, which is one of the main driving engines of the price determination in a contracting

\footnotetext{
${ }^{11}$ See Mendelson and Tunca $(2007 a, b)$ for models that make spot price and contracting endogenous to each other.
} 
process. On the other hand, there is usually much less information asymmetry on firms' discount rates or cost of capital. As we mentioned in Section 5, often, firms can borrow from banks at the ongoing publicly available common rate called "Bank Prime Loan Rate", plus/minus a few percentage points, and the precise rate usually depends on the firm size. Therefore, firms usually have a pretty good idea about each others' discount rates when they engage in contracting. Furthermore, for public companies, loan interest rates can be inferred from quarterly and annual statements. To summarize, firms' information about each others' cost of capital is usually much better than their information on others' firm-specific willingness-to-pay for a particular supplier's product, as the latter information is much more idiosyncratic and private, and, understandably, much better protected. Further, we believe that with uncertainty on the discount rates, our main results would be preserved and the insights (as we discussed in detail in Section $5)$ would continue to hold. Specifically, if the discount rates are uncertain, but the seller's discount rate is likely to be higher than that of the buyers' (i.e., if it is likely that $r_{S} \leq r_{B}$ ), the seller still would prefer to have sales contracts to maximize her discounted profits by collecting the fees at the time of the contract. On the flip side, if the buyer's discount rate is likely to be higher than the seller's, the seller would prefer to shift the payments to the future, since that would make the contracts attractive to the buyer and maximize the seller's profits. If the two discount rate distributions were somewhere in the middle, an outcome that is a mixture of these two polar cases would likely emerge. A detailed exploration of the effects of information asymmetry about discount rates on contract structure could be a potential future research direction as well.

In the general contract structure we study, the reservation price can be volume-dependent, while the unit exercise price $w$ is constant. There are several reasons for that. First, this reflects the common current practice in options contract structure. Highly complex options contract structures that make both reservation prices and exercise prices volume-dependent are not commonly observed in practice. Second, making the exercise price volume-dependent would add enormous complexity to the model and make the analysis intractable. Third, even if one could analyze such contracts, it is unclear how much the extra complexity given by making the exercise price volume-dependent will add to the efficiency of the contracts for most cases. The contracts we study, with fully free reservation price functional form $(R(\cdot))$, and endogenous exercise price $w$, have substantial flexibility, and much of the flexibility brought about by making the exercise price non-linear can be captured by the non-linearity of the reservation prices.

In our analysis we also included separate individual discount rates for the buyer and the seller. The discount rate for a given company is determined by many factors, including firm size, its credit rating, riskiness of the firm's current investments, and the balance of its assets and liabilities. In a general sense, these rates would be determined by combining market-wide factors with firm-specific factors, and by various methods including market portfolio-based methods (such as CAPM) and macroeconomic analysis. The determination of these discount rates are well beyond the scope of our current paper. However, it could be interesting to explore the links between discount rate determination and contracts in a future study that particulary focuses on the interaction of market, industry and firm-specific factors. 
Another extension direction for our model is to study alternative contracting structures. One such structure that is studied in both economics and operations management literatures for mitigating the inefficiencies of vertical disintegration is the two-part tariff (see, e.g., Schmalensee 1981, Tirole 1990, Cachon 1998). This structure involves a constant fee charged alongside a constant unit price for variable quantity. Provided that the setting is sufficiently simple, e.g., when there are no uncertainties, no hidden actions, or no downstream oligopolistic competition in the supply chain, such contracts can in fact be successful in coordinating the supply chain or achieving high contract efficiency. However, it is also known that with additional complexities two-part tariff contracts can prove to be inferior to other contract forms, but hybrid two-part-tariff-like contract structures can be built into or combined with other contract elements to improve supply chain efficiency (see, e.g., Dana and Spier 2001 and Cachon and Lariviere 2005). Another contracting structure that can be explored is Nash Bargaining. Dong and Liu (2007) present the analysis of forward contracting in the presence of spot markets under Nash bargaining. Extending their analysis to option contracts could be an interesting avenue for future research.

As in many other studies that analyze pricing and contracting for procurement, our model focuses on continuously differentiable functions for optimal contracts (see, e.g., Schmalensee and Willig 1989, Dasgupta and Spulber 1989/90, Tunca and Wu 2009). Naturally, in practice, contracts are implemented in simpler forms, such as piece-wise linear price schedules. A well-designed piece-wise linear contract would approximate the globally optimal continuously differentiable contract relatively closely. To that end, the higher the number of segments in the contract, the better the approximation will be. However, too many segments would make a contract too complex and less practically employable. Therefore the performance of such contracts are determined by the number of segments the seller can practically offer to a buyer. The piece-wise linear approximation of differentiable non-linear contract functions should normally not alter the main qualitative insights from the analysis. ${ }^{12}$

Again similar to most related models in the literature, we assumed that the demand and spot price distributions are independent. This assumption was necessary for the tractability of the general model with random $\theta$. However, for the benchmark case of fixed $\theta$ we studied in Section 4 , we can introduce this correlation without destroying tractability. We provide this analysis in Section B in the Online Supplement. Further, we also study the special cases of perfect positive and negative correlation in the general model in Section C in the Online Supplement. A main observation from these analyses is that correlation between consumer demand and spot price does not significantly affect the nature of the main insights of our study. The reason for this is that the assumption of independence of $s$ and $D$ distributions is not a main driver of the results we present in this paper. As we have explained in detail in Section 5, the relationships among the production cost, expected spot price, and buyer's and seller's discount rates determine the main contract structures. Specifically, if the seller has a higher discount rate than the buyer, it tends to be optimal for the seller to offer sales contracts that guarantee the payments and shifts them earlier in time. If the buyer has a higher discount rate, it tends to be optimal for the seller to offer option contracts so

\footnotetext{
${ }^{12}$ In certain "knife-edge" cases, in the optimal contracts in our model the reservation price can be linear. Such cases can emerge for parameter and distribution combinations that solve the differential equation (17) with equality.
} 
the buyer can find the contracts attractive as he benefits from controlling the risk by purchasing only the units he needs and also shifting the payments to the future. If the production costs are low compared to the expected salvage value of the units at the spot price, then it tends to be optimal for the seller to offer a large quantity to the buyer at a fixed price. On the other hand, if the production costs are large, it is best to make the contract offer contingent on the buyer's (random) valuation of the contract to eliminate potential excess costly production. A future study that analyzes the general correlation in the full model with uncertainty of the buyer's willingness-to-pay for the supplier's product can be another interesting extension.

In our model we also assumed that $p>\bar{s}+\bar{\theta}$, guaranteeing that it is always profitable to sell to the consumer market and that the buyer is always better off selling his product to the consumer market rather than to the spot market (since $p>\bar{s}+\bar{\theta}$ implies $p>\bar{s}(1-\phi)$ ). Again, one reason for this assumption is tractability - without this assumption, the analysis becomes very complex and intractable. However, beyond tractability, in our experience, the situations where the spot wholesale purchases are costlier than the consumer prices or where it is more profitable to sell to the spot market than to the consumer market are quite rare and happen only in exceptional cases and circumstances. Such cases often signal flaws in market design or unusual market manipulation caused by ill-conceived regulatory attempts (see, e.g., Wilson 2002 on the design flaws that contributed to the ultimate failure of the now-defunct California Power Exchange). Crossovers are not normal in the day-to-day operation of healthy markets. Thus, the assumption that the buyer always prefers to sell to the consumer market instead of to the wholesale spot market is a reasonable one to make within the scope of our model. Nevertheless, one direction for future research could be exploring cases where the consumer price can cross-over with the spot wholesale price, and studying the effects of such potential cross-over.

For tractability, we also assumed a fixed spot bid-ask spread coefficient $\phi$. The spread in spot trading can in fact depend on a number of market factors such as demand and supply in the spot market. However, although the existence of a bid-ask spread is necessary for our results, its particular form is not a major driver of our main qualitative results and insights. Further, the details of how the spread in the spot market gets determined moves the current model away from its main focus. However, extending the model to a case where the spread can functionally depend on other model elements and parameters could be an interesting subject for a future study.

\section{Concluding Remarks}

In this paper we studied the pricing problem of contracts for a supplier of an industrial good in the presence of spot trading and under information asymmetry about the buyer's valuation premium for the supplier's product. We jointly studied the questions: (i) When is it optimal to offer sales contracts versus options contracts? (ii) When is it optimal to offer flat fee versus volume-dependent contracts? and (iii) When does the optimal contract involve volume discounts and when does it involve volume premia? We derived 
the optimal non-linear pricing of procurement options contracts, derived the structure of the contract agreements, and studied the determinants of pricing and contract characteristics.

Our goal has been to provide an approach to the design of procurement contracts combining buyers' private valuations, spot market trading, information asymmetry, and general pricing structures for option contracts. To this end, we analyzed a solution for a general class of option contracts that encompasses a broad spectrum of procurement contract structures commonly employed in practice. Our analysis aims to provide insights for the endogenously determined nature of procurement contracts, and to build links between variables such as spot price distribution and bid-ask spread of the spot market and procurement contract characteristics, which were not explored previously in the literature. We hope that these findings provide a better understanding of complex non-linear procurement contracts and can help contribute to future studies that continue to build a more generalized theory of procurement agreements.

\section{References}

Ashbaugh-Skaife, H. and R. LaFond (2006). Firms corporate governance and the cost of debt: An analysis of U.S. firms GMI ratings. Working Paper, University of Wisconsin, Madison.

Baker, G., R. Gibbons, and K. J. Murphy (2002). Relational contracts and the theory of the firm. Quarterly Journal of Economics 117(1), 39-83.

Barnes-Shuster, D., Y. Bassok, and R. Anupindi (2002). Coordination and flexibilty in supply contracts with options. MESSOM 4(3), 171-207.

Berk, J. and P. DeMarzo (2007). Corporate Finance (1 ed.). Boston MA: Pearson.

Brown, A. and H. Lee (1998). The win-win nature of options based capacity reservation arrangements. Vanderbilt University working paper.

Cachon, G. (1998). Competitive supply chain inventory management. In S. Tayur, R. Ganeshan, and M. Magazine (Eds.), Quantitative Models for Supply Chain Management. Boston: Kluwer.

Cachon, G. (2003). Supply chain coordination with contracts. In S. Graves and T. de Kok (Eds.), Handbooks in Operations Research and Management Science: Supply Chain Management. Amsterdam: Elsevier.

Cachon, G. and M. Lariviere (2005). Supply chain coordination with revenue sharing contracts. Management Science 51(1), 30-44.

Chen, F. (2003). Information sharing and supply chain coordination. In A. de Kok and S. Graves (Eds.), Handbooks in Operations Research and Management Science, Vol. 11, Supply Chain Management: Design, Coordination, and Operation. Amsterdam: Elsevier. 
Dana, J. D. and K. E. Spier (2001). Revenue sharing and vertical control in the video rental industry. Journal of Industrial Economics 49(3), 223-245.

Dasgupta, S. and D. Spulber (1989/90). Managing procurement auctions. Information Economics and Policy 4, 5-29.

Dong, L. and E. Durbin (2005). Markets for surplus components with a strategic supplier. Naval Research Logistics 52(8), 734-753.

Dong, L. and H. Liu (2007). Equilibrium forward contracts on nonstorable commodities in the presence of market power. Operations Research 55(1), 128-145.

Donohue, K. (2000). Efficient supply contracts for fashion goods with forecast updating and two production modes. Management Science 46(11), 1397-1411.

Duffie, D. (1996). Dynamic Asset Pricing Theory (Second ed.). Princeton, NJ: Princeton University Press.

Eppen, G. D. and A. V. Iyer (1997). Backup agreements in fashion buying - the value of upstream flexibility. Management Science 43(11), 1469-1484.

Fudenberg, D. and J. Tirole (1991). Game Theory. MIT Press.

Grey, W., T. Olavson, and D. Shi (2005). The role of e-marketplaces in relationship-based supply chains. IBM Systems Journal 44(1), 109-123.

Hail, L. and C. Leuz (2009). Cost of capital effects and changes in growth expectations around u.s. cross-listings. Journal of Financial Economics 93(3), 428 - 454.

Hart, O. D. and J. Moore (1988). Incomplete contracts and renegotiation. Econometrica 56, 755-785.

Katz, P., A. Sadrian, and P. Tendick (1994). Telephone companies analyze price quotations with bellcore PDSS software. Interfaces 24, 50-63.

Kleindorfer, P. R. and D. Wu (2003). Integrating long- and short-term contracting via business-tobusiness exchanges for capital-intensive industries. Management Science 49(11), 1597-1615.

Lee, H. S. and S. Whang (2002). The impact of the secondary market on the supply chain. Management Science 48(6), 719-731.

Levi, M., P. Kleindorfer, and D. Wu (2003). Codifiability, relationship-specific Information Technology investment, and optimal contracting. Journal of Management Information Systems 20(2), 77-98.

Malone, T. W., J. Yates, and R. I. Benjamin (1987). Electronic markets and electronic hierarchies. Communications of the ACM 30(6), 484-497.

Martinez-de-Albeniz, V. and D. Simchi-Levi (2005a). Mean-variance trade-offs in supply contracts. Working Paper, OR Center, MIT. 
Martinez-de-Albeniz, V. and D. Simchi-Levi (2005b). A portfolio approach to procurement contracts. Production and Operations Management 14(1), 90-114.

Martinez-de-Albeniz, V. and D. Simchi-Levi (2008). Competition in the supply option market. Operations Research 57, 1082-1097.

Mas-Colell, A., M. D. Whinston, and J. R. Green (1995). Microeconomic Theory. Oxford University Press.

Mendelson, H. and T. I. Tunca (2007a). Business-to-Business Electronic Markets: Does trading on new information create value and for whom? In U. M. Apte and U. S. Karmarkar (Eds.), Managing in the Information Economy: Current Research Issues, Chapter 9, pp. 209-230. Norwell, MA: Kluwer Academic Publishers.

Mendelson, H. and T. I. Tunca (2007b). Strategic spot trading in supply chains. Management Science 53(5), 742-759.

Myerson, R. B. (1981). Optimal auction design. Mathematics of Operations Research 6, 58-73.

Peleg, B., H. S. Lee, and W. Hausman (2002). Short-term e-procurement strategies vs. long-term contracts. Production and Operations Management 11(4), 458-479.

Porteus, E. L. (2002). Foundations of Stochastic Inventory Theory. Stanford, CA: Stanford University Press.

Robertson, J. (2002, July 5). Buyers enjoy fire sale in 256Mbit DRAMs. EBN.

Schmalensee, R. (1981). Monopolistic two-part pricing arrangements. The Bell Journal of Economics 12(2), 445-466.

Schmalensee, R. and R. Willig (1989). Handbook of Industrial Organization, Volume 1. Amsterdam: North-Holland.

Shin, H. and T. I. Tunca (2010). Do firms invest in forecasting efficiently? The effect of competition on demand forecast investments and supply chain coordination,. Forthcoming, Operations Research.

Tirole, J. (1990). The Theory of Industrial Organization. Cambridge, MA: The MIT Press.

Tsay, A. (1999). Quantity-flexibility contract and supplier-customer incentives. Management Science 45(10), 1399-1358.

Tunca, T. I. (2008). Information precision and asymptotic efficiency of industrial markets. Journal of Mathematical Economics 44(9-10), 964-996.

Tunca, T. I. and Q. Wu (2009). Multiple sourcing and procurement process selection with bidding events. Management Science 55(5), 763-780. 
Tunca, T. I. and S. A. Zenios (2006). Supply auctions and relational contracts for procurement. MESOM 8(1), 43-67.

U.S.F.R.B. (2008). Bank prime loan rate changes: Historical dates of changes and rates. Available at http://research.stlouisfed.org/fred2/data/PRIME.txt.

Williamson, O. E. (1981a). The economics of organization: The transaction cost approach. American Journal of Sociology 87(3), 548-575.

Williamson, O. E. (1981b). The modern corporation: Origins, evolution, attributes. Journal of Economic Literature 19(4), 1537-1568.

Wilson, R. (2002). Architecture of power markets. Econometrica 70(4), 1299-1340.

Wilson, R. B. (1993). Nonlinear pricing. New York: Oxford University Press.

Wu, D. and P. Kleindorfer (2005). Competitive options, supply contracting, and electronic markets. Management Science 51(3), 452-466.

Wu, D., P. R. Kleindorfer, and J. E. Zhang (2002). Optimal bidding and contracting strategies for capital-intensive goods. European Journal of Operational Research 137(3), 657-676. 

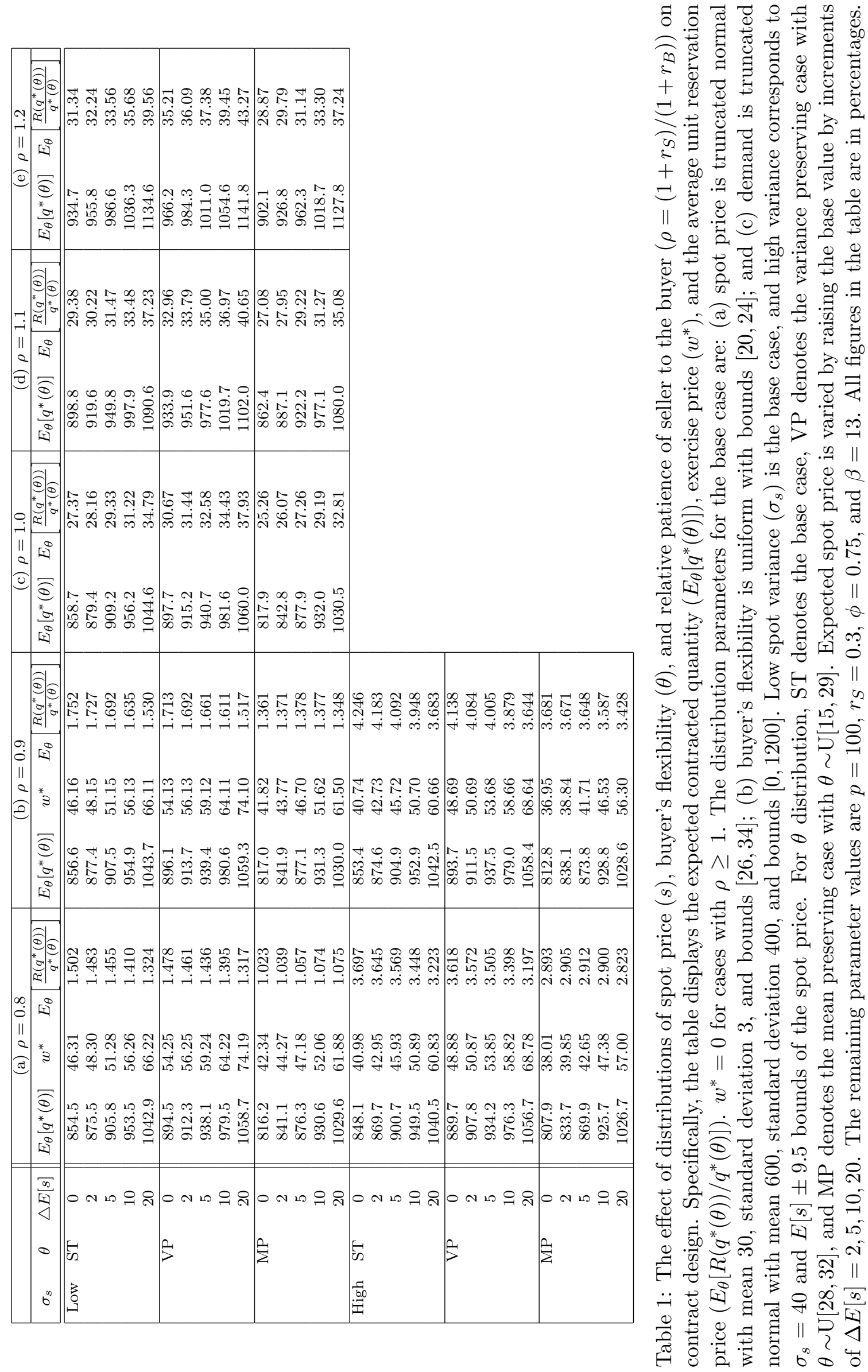


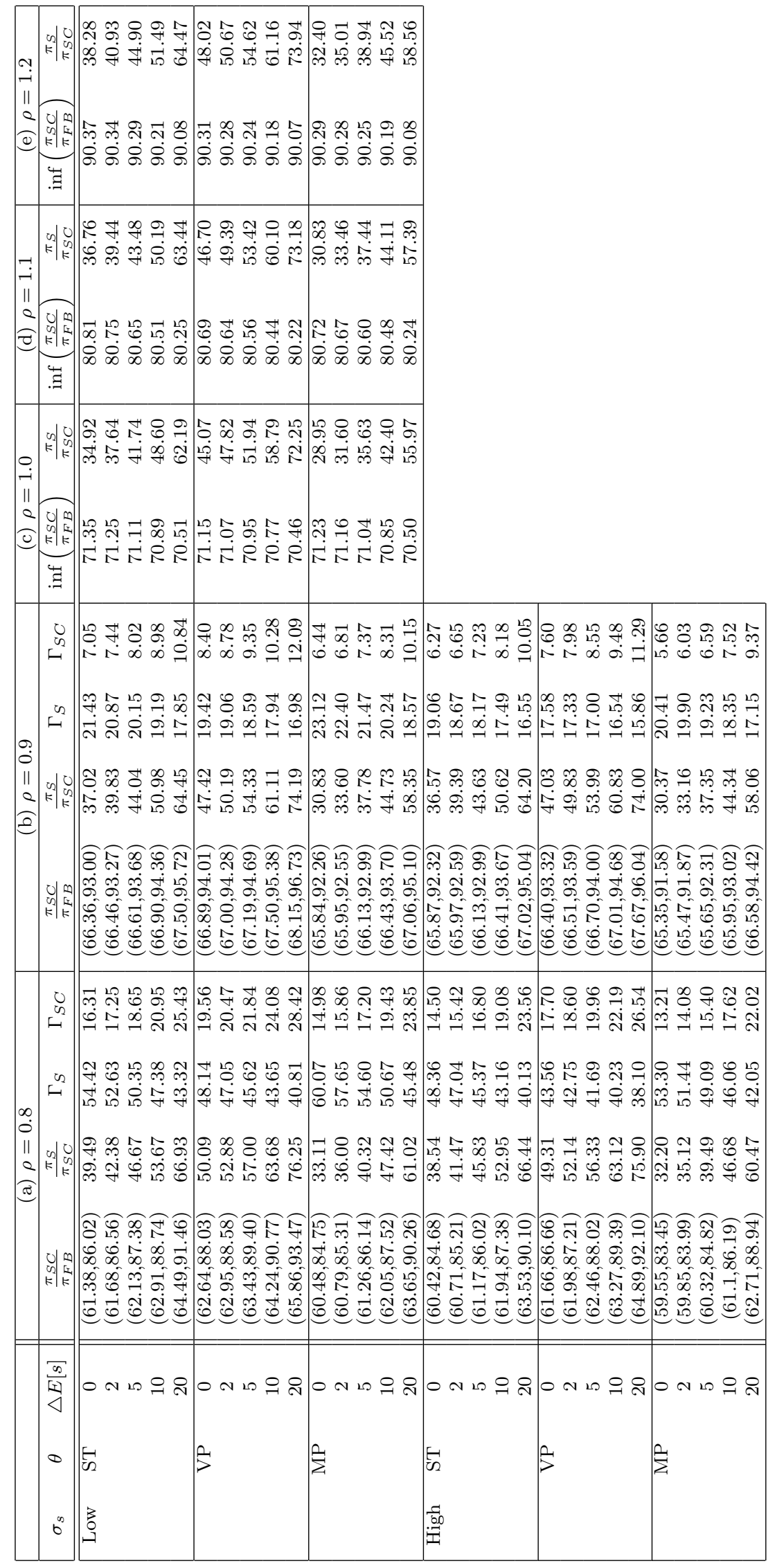

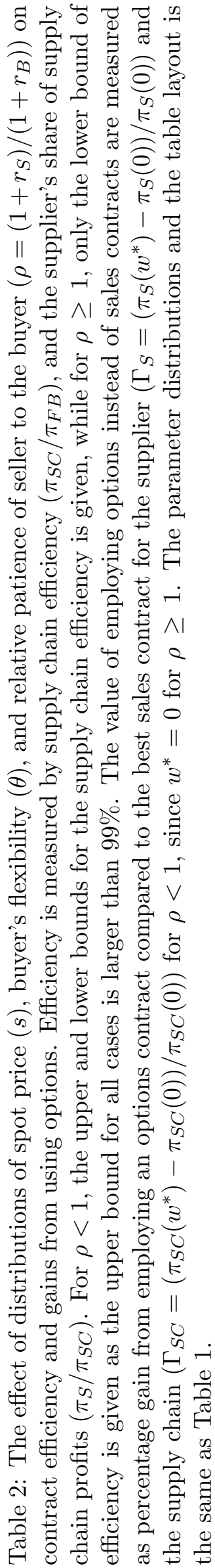




\section{Online Supplement for}

\section{"Sourcing Flexibility, Spot Trading, and Procurement Contract Structure" Pamela Pen-Erh Pei, David Simchi-Levi, and Tunay I. Tunca}

\section{A Proofs of Propositions}

Proof of Proposition 1: In solving the optimization problem of the supplier, we will eliminate the buyer's IR constraint and substitute it into the the seller's objective function, and solve the seller's unconstrained problem. The solution will then unroll by plugging back the unconstrained optimizer into the seller's expected profit expression.

First note that since $\theta$ is fixed, the seller knows the buyer type perfectly, and hence can extract the full buyer surplus by offering a single quantity-price combination, which eliminates the buyer's IC constraint. That is, in the optimal contract $\hat{\pi}_{B}(q, w, \theta)=0$, and $\pi_{S}(q, w)=R(q, w)+V(q, w, \theta)=$ $-\hat{\pi}_{B}(q, w, \theta)+\varphi(q, w, \theta)+V(q, w)=\frac{1}{1+r_{B}} \varphi(q, w, \theta)+V(q, w)$. Now,

$$
\begin{aligned}
\frac{\varphi(q, w)}{1+r_{B}}+V(q, w)= & \frac{1}{1+r_{B}}\left(E_{s}\left[(s+\theta-w)^{+}\right] E_{D}[\min (D, q)]+E_{s}\left[(s(1-\phi)-w)^{+}\right] E_{D}\left[(q-D)^{+}\right]\right) \\
& +\frac{1}{1+r_{S}}\left(\left(E[s](1-\phi)-\left(1+r_{S}\right) \beta-E_{s}\left[(s(1-\phi)-w)^{+}\right]\right) q\right. \\
& \left.\quad+\int_{w-\theta}^{\frac{w}{1-\phi}}(w-s(1-\phi)) E_{D}[\min (D, q)] d F_{s}(s)\right)+\left(\frac{E[s](1-\phi)}{1+r_{S}}-\beta\right)^{+} \cdot(K-q) \\
= & \left.\frac{1}{1+r_{S}}\left(\left(E[s](1-\phi)-\left(1+r_{S}\right)\right) \beta\right) q+\int_{w-\theta}^{\bar{s}}(\phi s+\theta) E_{D}[\min (D, q)] d F_{s}(s)\right) \\
& +\frac{r_{S}-r_{B}}{\left(1+r_{S}\right)\left(1+r_{B}\right)}\left(E_{s}\left[(s+\theta-w)^{+}\right] E_{D}[\min (D, q)]\right. \\
& \left.\quad+E_{s}\left[(s(1-\phi)-w)^{+}\right] E_{D}\left[(q-D)^{+}\right]\right)+\left(\frac{E[s](1-\phi)}{1+r_{S}}-\beta\right)^{+} \cdot(K-q) .(\mathrm{A} .1)
\end{aligned}
$$

Therefore, relaxing the IR constraint for the seller momentarily, writing the first and second derivatives,

$$
\begin{aligned}
& \frac{d(\varphi(q, w)+V(q, w))}{d q}= \frac{1}{1+r_{S}}\left(E[s](1-\phi)-\left(1+r_{S}\right) \beta+\int_{w-\theta}^{\bar{s}}(\phi s+\theta) \bar{F}_{D}(q) d F_{s}(s)\right) \\
&+\frac{r_{S}-r_{B}}{\left(1+r_{S}\right)\left(1+r_{B}\right)}\left(\int_{w-\theta}^{\bar{s}}(s+\theta-w) \bar{F}_{D}(q) d F_{s}(s)\right. \\
&\left.\quad+\int_{\frac{w}{1-\phi}}^{\bar{s}}(s(1-\phi)-w) F_{D}(q) d F_{s}(s)\right)-\left(\frac{E[s](1-\phi)}{1+r_{S}}-\beta\right)^{+}
\end{aligned}
$$


and

$$
\begin{aligned}
\frac{d^{2}(\varphi(q, w)+V(q, w))}{d q^{2}}= & -\frac{1}{1+r_{S}} \int_{w-\theta}^{\bar{s}}(\phi s+\theta) f_{D}(q) d F_{s}(s) \\
& -\frac{r_{S}-r_{B}}{\left(1+r_{S}\right)\left(1+r_{B}\right)}\left(\int_{w-\theta}^{\frac{w}{1-\phi}}(s+\theta-w) f_{D}(q) d F_{s}(s)\right. \\
& \left.+\int_{\frac{w}{1-\phi}}^{\bar{s}}(\phi s+\theta) f_{D}(q) d F_{s}(s)\right) .
\end{aligned}
$$

Note that $\left(r_{S}-r_{B}\right) /\left(\left(1+r_{S}\right)\left(1+r_{B}\right)\right) \geq-1 /\left(1+r_{S}\right)$. Therefore, by (A.3),

$$
\frac{d^{2}(\varphi(q, w)+V(q, w))}{d q^{2}} \leq-\frac{1}{1+r_{S}} \int_{w-\theta}^{\frac{w}{1-\phi}}(w-s(1-\phi)) f_{D}(q) d F_{s}(s) \leq 0 .
$$

That is, the objective function is concave, and hence in optimality, either the first order condition is satisfied or $q^{*}(w)=K$. Further, since $q=0$ is a feasible point, concavity of $\varphi(q, w)+V(q, w)$ in $q$ implies that the IR constraint for the seller is satisfied as well. Therefore, by equating (A.2) to zero, and bounding the optimum by $K$, the optimal reservation quantity $q^{*}(w)$ is given as in (7). Further, since $\hat{\pi}_{B}\left(q^{*}(w), w, \theta\right)=-R\left(q^{*}(w), w\right)+\varphi\left(q^{*}(w), w\right)=0$, the optimal reservation price $R\left(q^{*}(w), w\right)$ for a given $w \geq 0$ can then be found by plugging $(7)$ in $\varphi\left(q^{*}(w), w\right)$, yielding (8). This completes the proof for part (i).

The sketch of the proof for part (ii) is as follows: We first calculate the derivative of $\pi_{S}\left(w, R\left(q^{*}(w), w\right)\right)$ with respect to $w$. Then we evaluate the derivative at relevant regions to derive the shape of the profit function as a function of $w$, which brings us to the conclusions.

By (A.1), and plugging in (7), we have

$$
\begin{aligned}
\frac{d \pi_{S}(w)}{d w}= & \frac{\partial \pi_{S}(w)}{\partial w}+\frac{d \pi_{S}(w)}{d q^{*}(w)} \cdot \frac{d q^{*}(w)}{d w} \\
= & -\frac{1}{1+r_{S}}(\phi(w-\theta)+\theta) E_{D}\left[\min \left(D, q^{*}(w)\right)\right] \cdot f_{s}(w-\theta) \\
& -\frac{r_{S}-r_{B}}{\left(1+r_{S}\right)\left(1+r_{B}\right)}\left(\int_{w-\theta}^{\bar{s}} E_{D}\left[\min \left(D, q^{*}(w)\right)\right] d F_{s}(s)+\int_{\bar{w}}^{\bar{s}} E_{D}\left[\left(q^{*}(w)-D\right)^{+}\right] d F_{s}(s)\right)
\end{aligned}
$$

First, consider the case where $r_{B}<r_{S}$. In this case, both terms in (A.5) are negative for all $w \geq 0$. Therefore, the optimal $w$ for the buyer is 0 and optimal contracts are sales contracts. Now suppose $r_{B}=r_{S}$. In this case $d \pi_{S}(w) / d w=-1 /\left(1+r_{S}\right)(\phi(w-\theta)+\theta) E_{D}\left[\min \left(D, q^{*}(w)\right)\right] \cdot f_{s}(w-\theta)<0$ for $\underline{s}+\theta<w<\bar{s}+\theta$, and $\frac{d \pi_{S}(w)}{d w}=0$ otherwise. Therefore, there is a continuum of optimal contracts, for $w^{*} \in[0, \underline{s}+\theta]$, and both sales $\left(w^{*}=0\right)$ and options contracts $\left(w^{*}>0\right)$ can prevail. Finally, when $r_{B}>r_{S}$, on $w \in[0, \underline{s}+\theta]$, the second term in (A.5) is strictly positive, while the first term is zero. Therefore $\pi_{S}(w)$ is strictly increasing on $w \in[0, \underline{s}+\theta)$. Further, for $w>\bar{s}+\theta$, all terms vanish in (A.5), and $d \pi_{S}(w) / d w=0$. Therefore, for $r_{B}>r_{S}, \pi_{S}(w)$ is strictly increasing on $[0, \underline{s}+\theta)$, and constant on $w>\bar{s}+\theta$. Since $\pi_{S}(w)$ 
is continuous, it is maximized on $w \in[\underline{s}+\theta, \bar{s}+\theta]$. Finally, for sufficiently small $r_{B}-r_{S}$,

$$
\begin{aligned}
\frac{1}{1+r_{S}}(\phi \underline{s} & +\theta) E_{D}\left[\min \left(D, q^{*}(\underline{s}+\theta)\right)\right] \cdot f_{s}(\underline{s}) \\
& >\frac{r_{B}-r_{S}}{\left(1+r_{S}\right)\left(1+r_{B}\right)}\left(\int_{\underline{s}}^{\bar{s}} E_{D}\left[\min \left(D, q^{*}(w)\right)\right] d F_{s}(s)+\int_{\underline{\underline{s}}+\theta}^{\bar{s}} E_{D}\left[\left(q^{*}(w)-D\right)^{+}\right] d F_{s}(s)\right) .
\end{aligned}
$$

Therefore, by (A.5), $d \pi_{S}(w) /\left.d w\right|_{w=\underline{s}+\theta}<0$. Since for $r_{B}$ sufficiently large $\pi_{S}$ is monotonically nondecreasing in $w$, and $\pi_{S}$ is continuous in $r_{B}$, it follows that there exists $\delta_{r}>0$ such that when $r_{S}<r_{B}<$ $r_{S}+r_{B}, \pi_{S}$ is non-monotonic in $w$ and $w^{*}<\bar{s}+\theta$. This completes the proof.

Proof of Lemma 1: We will show that $G$ takes values between 0 and $\left(\left(1+r_{S}\right) \beta-E[s](1-\phi)\right)^{+} /(\rho-1)$ on $[0, \bar{s}(1-\phi)]$, and combine it with the fact that it is continuous and monotonically decreasing to derive the conclusion. First, notice that $\beta \leq \frac{E[s](1-\phi)}{1+r_{B}}$ implies $\rho E[s](1-\phi) \geq\left(1+r_{S}\right) \beta$, which in turn implies $G(0) \geq\left(\left(1+r_{S}\right) \beta-E[s](1-\phi)\right)^{+} /(\rho-1)$, since $G(0)=E[s](1-\phi)$. Further, $G(w)=0$ for all $w \geq \bar{s}(1-\phi)$. Combining this with the fact that $G$ is strictly decreasing for $0 \leq w \leq \bar{s}(1-\phi)$ and $\left(\left(1+r_{S}\right) \beta-E[s](1-\phi)\right)^{+} /(\rho-1)>0$, the statement follows.

Proof of Proposition 2: The outline of the proof is as follows: We first characterize the solution of the buyer's IC constraints, and using that show that all of the buyer's IR constraints are satisfied if and only if the IR constraint for the type $\underline{\theta}$ is satisfied. This allows us to eliminate all of the buyer's IR constraints except for type $\underline{\theta}$, which we show must be binding. Next, utilizing the characterization of the solution of buyer's IC constraints, we substitute for the value of the seller's expected revenue implied by these constraints into the seller's objective function. This allows us to convert the seller's optimization problem to an equivalent one without the buyer's IC constraints. We next temporarily relax the seller's IR constraint, and show that the seller's problem can be solved pointwise for the quantity chosen for each type of buyer from the offered menu, $q^{*}(w, \theta)$. We finally prove the conditions for flat-price and volume dependent contract offers, and calculate the price that corresponds to each quantity, $R\left(q^{*}(w, \theta), w\right)$.

First, for a given price-quantity menu $R(q(w, \theta), w))$, in order type $\theta$ buyer to indeed choose the quantity, $q(w, \theta)$, meant for him, i.e., in order for the $I C$ constraint to be satisfied, the first order condition $d \hat{\pi}_{B}(q, w, \theta) /\left.d q\right|_{q=q(w, \theta)}=0$ must hold for all $\theta \in[\underline{\theta}, \bar{\theta}]$. By the envelope theorem, we then have

$$
\begin{aligned}
\left.\frac{d \hat{\pi}_{B}(q, w, \theta)}{d \theta}\right|_{q=q(w, \theta)} & =\left.\frac{\partial \hat{\pi}_{B}(q, w, \theta)}{\partial \theta}\right|_{q=q(w, \theta)}+\left.\frac{d \hat{\pi}_{B}(q, w, \theta)}{d q}\right|_{q=q(w, \theta)} \cdot \frac{d q(w, \theta)}{d \theta} \\
& =\left.\frac{\partial \hat{\pi}_{B}(q, w, \theta)}{\partial \theta}\right|_{q=q^{*}(w, \theta)} \geq 0 .
\end{aligned}
$$

Therefore,

$$
\hat{\pi}_{B}(q, w, \theta)=\left.\int_{\underline{\theta}}^{\theta} \frac{\partial \hat{\pi}(q, w, a))}{\partial a}\right|_{q=q(w, a)} d a,
$$


and in any given feasible solution to $(12), \hat{\pi}_{B}(q(w, \theta), w, \theta)$ has to be increasing in $\theta$. Therefore, the second set of conditions in (12) are satisfied if and only if $\hat{\pi}_{B}(q(w, \underline{\theta}), w, \underline{\theta}) \geq 0$. Further, in the optimal schedule, this constraint should be binding, i.e., $\hat{\pi}_{B}(q(w, \underline{\theta}), w, \underline{\theta})=0$, since otherwise, the seller can increase the payment charged to all types without violating their IR constraints.

Now, $\partial \hat{\pi}_{B}(q, w, \theta) / \partial \theta=\partial\left(\varphi(q, w, \theta) /\left(1+r_{B}\right)-R(q, w)\right) / \partial \theta=(\partial \varphi(q, w, \theta) / \partial \theta)\left(1+r_{B}\right)^{-1}$. Then by (A.7), and applying integration by parts, we have

$$
\begin{aligned}
\int_{\underline{\theta}}^{\bar{\theta}} R(q(w, \theta), w) d F_{\theta}(\theta)= & \int_{\underline{\theta}}^{\bar{\theta}}\left(\frac{1}{1+r_{B}} \varphi(q(w, \theta), w, \theta)-\hat{\pi}_{B}(q(w, \theta), w, \theta)\right) d F_{\theta}(\theta) \\
= & \frac{1}{1+r_{B}} \int_{\underline{\theta}}^{\bar{\theta}} \varphi(q(w, \theta), w, \theta) d F_{\theta}(\theta)-\hat{\pi}_{B}(q(w, \underline{\theta}), w, \underline{\theta}) \\
& -\left.\frac{1}{1+r_{B}} \int_{\underline{\theta}}^{\bar{\theta}} \int_{\underline{\theta}}^{\theta} \frac{\partial \varphi(q, w, a)}{\partial a}\right|_{q=q(w, a)} d a d F_{\theta}(\theta) \\
= & \frac{1}{1+r_{B}}\left\{\int_{\underline{\theta}}^{\bar{\theta}} \varphi(q(w, \theta), w, \theta) d F_{\theta}(\theta)-\left.\left(\left.F_{\theta}(\theta) \int_{\underline{\theta}}^{\theta} \frac{\partial \varphi(q, w, a)}{\partial a}\right|_{q=q(w, a)} d a\right)\right|_{\underline{\theta}} ^{\bar{\theta}}\right. \\
\left.\quad+\left.\int_{\underline{\theta}}^{\bar{\theta}} F_{\theta}(\theta) \frac{\partial \varphi(q, w, \theta)}{\partial \theta}\right|_{q=q(w, \theta)} d \theta\right\} & \frac{1}{1+r_{B}} \int_{\underline{\theta}}^{\bar{\theta}}\left(\varphi(q(w, \theta), w, \theta)-\left.\frac{\bar{F}_{\theta}(\theta)}{f_{\theta}} \frac{\partial \varphi(q, w, \theta)}{\partial \theta}\right|_{q=q(w, \theta)}\right) d F_{\theta}(\theta) .
\end{aligned}
$$

Plugging (A.9) in the objective function in (12) we obtain

$$
\pi_{S}(w)=\int_{\underline{\theta}}^{\bar{\theta}}\left(V(q(w, \theta), w, \theta)+\frac{1}{1+r_{B}}\left(\varphi(q(w, \theta), w, \theta)-g_{\theta}(\theta) \frac{\partial \varphi(q(w, \theta), w, \theta)}{\partial \theta}\right)\right) d F_{\theta}(\theta)
$$

where $g_{\theta}(\theta)=\bar{F}_{\theta}(\theta) / f_{\theta}(\theta)$. For notational convenience, define $H(q, w, \theta)$ as the integrand in (A.10). Notice that (A.10) can be optimized pointwise. For a constant $\theta$, the first derivative is

$$
\begin{aligned}
\frac{d H(q(w, \theta), w, \theta)}{d q(w, \theta)}=- & \left(\left(1+r_{S}\right) \beta-E[s](1-\phi)\right)^{+}+(\rho-1) \int_{\frac{w}{1-\phi}}^{\bar{s}}(s(1-\phi)-w) d F_{s}(s) \\
& \quad+\bar{F}_{D}(q(w, \theta))\left\{\int_{w-\theta}^{\bar{s}}\left(\phi s+\theta-g_{\theta}(\theta)\right) d F_{s}(s)\right. \\
& \left.+(\rho-1)\left(\int_{w-\theta}^{\bar{s}}\left(s+\theta-g_{\theta}(\theta)-w\right) d F_{s}(s)-\int_{\frac{w}{1-\phi}}^{\bar{s}}(s(1-\phi)-w) d F_{s}(s)\right)\right\} .
\end{aligned}
$$

Equating (A.11) to zero and solving for $q(w, \theta)$ yields

$$
q^{*}(w, \theta)=\bar{F}_{D}^{-1}\left(\frac{\left(\left(1+r_{S}\right) \beta-E[s](1-\phi)\right)^{+}-(\rho-1) G(w)}{\eta(w, \theta)-(\rho-1) G(w)}\right)
$$


where

$$
\eta(w, \theta)=\int_{w-\theta}^{\bar{s}}\left(\phi s+\theta-g_{\theta}(\theta)\right) d F_{s}(s)+(\rho-1) \int_{w-\theta}^{\bar{s}}\left(s+\theta-g_{\theta}(\theta)-w\right) d F_{s}(s),
$$

and $G$ is as defined in Section 5.1. Next we show the concavity of $H$ for the desired range of $\rho$. From (A.11), the second derivative of $H$ with respect to $q(w, \theta)$ is

$$
\frac{d^{2} H(q(w, \theta), w, \theta)}{d q(w, \theta)^{2}}=-f_{D}(q(w, \theta))(\eta(w, \theta)-(\rho-1) G(w))
$$

Now define

$$
\begin{aligned}
\varrho(w, \theta) \triangleq & \eta(w, \theta)-(\rho-1) G(w) \\
= & \int_{w-\theta}^{\bar{s}}\left(\phi s+\theta-g_{\theta}(\theta)\right) d F_{s}(s) \\
& +(\rho-1)\left(\int_{w-\theta}^{\bar{s}}\left(s+\theta-g_{\theta}(\theta)-w\right) d F_{s}(s)-\int_{\frac{w}{1-\phi}}^{\bar{s}}(s(1-\phi)-w) d F_{s}(s)\right) .
\end{aligned}
$$

Taking the derivative of $\varrho$ with respect to $w$,

$$
\frac{d \varrho(w, \theta)}{d w}= \begin{cases}-(\rho-1) \bar{F}_{s}\left(\frac{w}{1-\phi}\right) & \text { for } w<\underline{s}+\theta \\ -f_{s}(w-\theta) \nu(w, \theta) & \text { for } w \geq \underline{s}+\theta\end{cases}
$$

where

$$
\nu(w, \theta)=\phi(w-\theta)+\theta-g_{\theta}(\theta)+(\rho-1)\left(g_{s}(w-\theta)-g_{\theta}(\theta)\right),
$$

and $g_{s}(s)=\bar{F}_{s}(s) / f_{s}(s)$. Notice that

$$
\begin{aligned}
\nu(\underline{s}+\underline{\theta}, \underline{\theta}) & =\phi \underline{s}+\underline{\theta}-\frac{1}{f_{\theta}(\underline{\theta})}+(\rho-1)\left(\frac{1}{f_{s}(\underline{s})}-\frac{1}{f_{\theta}(\underline{\theta})}\right) \\
& \geq \phi \underline{s}+\underline{\theta}-\frac{1}{f_{s}(\underline{s})}>0,
\end{aligned}
$$

since $\rho>0$, and $(\phi \underline{s}+\underline{\theta})^{-1}<f_{s}(\underline{s}) \leq f_{\theta}(\underline{\theta})$. Now,

$$
\frac{d g_{s}(s)}{d s}=\frac{d}{d s} \frac{\bar{F}_{s}(s)}{f_{s}(s)}=-1-g_{s}(s) \frac{d f_{s}(s) / d s}{f_{s}(s)} .
$$

Since $g_{s}(s)$ is monotonically decreasing in $s$, finite at $\underline{s}$, and $d \log \left(f_{s}(s)\right) / d s$ is bounded on $[\underline{s}, \bar{s}]$,

$$
\left|\frac{d g_{s}(s)}{d s}\right|<\infty, \text { for all } s \in[\underline{s}, \bar{s}] .
$$

In addition, from (A.17), we also have

$$
\frac{d \nu(w, \theta)}{d w}=\phi+\left.(\rho-1) \frac{d g_{s}(s)}{d s}\right|_{s=w-\theta}
$$


which is nonnegative for $0<\rho<1+\frac{\phi}{\sup _{s \in[s, \bar{s}]}\left|\frac{d g_{s}(s)}{d s}\right|} \triangleq \bar{\rho}$, since $g_{s}(s)$ is decreasing in $s$, and where $\bar{\rho}>1$, by (A.20). Further

$$
\frac{d \nu(w, \theta)}{d \theta}=(1-\phi)-\rho \frac{d g_{\theta}(\theta)}{d \theta} \geq 0,
$$

since $g_{\theta}(\theta)$ is decreasing in $\theta$. It follows that $\nu(w, \theta) \geq 0$ for all $\theta$ and $w \geq \underline{s}+\theta$. Combining this with (A.16), it follows that, for $\rho<\bar{\rho}, d \varrho(w, \theta) / d w<0$ for $\underline{s}+\theta \leq w<\bar{s}+\theta$, and $d \varrho(w, \theta) / d w=0$ for $w \geq \bar{s}+\theta$, for all $\theta \in[\underline{\theta}, \bar{\theta}]$. From (A.16), it also follows that for $0 \leq w<\underline{s}+\theta, d \varrho(w, \theta) / d w<0$ if and only if $\rho<1$. But

$$
\varrho(0, \theta)=\rho\left(\phi E[s]+\theta-g_{\theta}(\theta)\right)>0,
$$

and

$$
\varrho(w, \theta)=0, \forall w \geq \bar{s}+\theta .
$$

Therefore $\varrho(w, \theta)>0$ for $0 \leq w<\bar{s}+\theta$. Combining this with (A.14) and (A.15), it follows that $H(q, w, \theta)$ is strictly concave for all $q$. Further, since $H(q, w, \theta)$ is strictly concave and $q(w, \theta)=0$ is feasible for all $\theta$, and yields zero profits for the seller, the IR constraint for the seller is satisfied as well. Therefore $q^{*}(w, \theta)$ as given in (14) is the pointwise optimum quantity for the seller to offer to type $\theta$ buyer. Hence, $\left(q^{*}(w, \theta), \theta\right),[\underline{\theta}, \bar{\theta}]$ is the optimal quantity menu for the seller's problem.

We can now derive the conditions for flat price and volume dependent contracts, and calculate the contract price $R\left(q^{*}(w, \theta), w\right)$. By (14), since $\varrho(w, \theta)>0$, we have $q^{*}(w, \theta)=\bar{D}$ if and only if $(\rho-1) G(w) \geq$ $\left(1+r_{S}\right) \beta-E[s](1-\phi)$. By Lemma 1 , given $\rho>1$ and $\beta \leq \frac{E[s](1-\phi)}{1+r_{B}}$, there exists a $w_{c}$ such that $(\rho-1) G\left(w_{c}\right)=\left(1+r_{S}\right) \beta-E[s](1-\phi)$, and since $G$ is strictly decreasing in $w$, for $0 \leq w \leq w_{c}$, $(\rho-1) G(w) \geq\left(1+r_{S}\right) \beta-E[s](1-\phi)$. This means that, when $\rho>1$ and $\beta \leq \frac{E[s](1-\phi)}{1+r_{B}}, q^{*}(w, \theta)=\bar{D}$ for all $\theta$, i.e., the seller offers a constant contract. The price of the contract follows by plugging $q^{*}(w, \theta)=\bar{D}$ in $\hat{\pi}(q(w, \theta), w, \underline{\theta})$ as $R(w)=\frac{1}{1+r_{B}} \varphi(\bar{D}, w, \underline{\theta})$ as stated. This proves part $(i)$.

For part $(i i)$, similar to above, $\varrho(w, \theta) \geq 0$ for any $(w, \theta)$. The optimal reservation price schedule is volume-dependent only when the seller is able to differentiate the buyer's type. By $(14), q^{*}(w, \theta)$ is dependent on $\theta$ if and only if $\eta(w)<\left(1+r_{S}\right) \beta-E[s](1-\phi)$, which can only happen if the flat price conditions are violated. Note that

$$
\frac{d \eta(w, \theta)}{d \theta}=\left(1-\rho g_{\theta}^{\prime}(\theta)\right) \bar{F}_{s}(w-\theta)+f_{s}(w-\theta) \nu(w, \theta),
$$

where $\nu(w, \theta)$ is as defined in (A.17). As we have shown above, $\nu(w, \theta) \geq 0$. Combining this with the fact that $g_{\theta}^{\prime}(\theta) \leq 0$, it follows that $d \eta(w, \theta) / d \theta \geq 0$, and $\eta(w, \theta)$ is increasing in $\theta$. Since $\eta(w, \theta)$ is increasing in $\theta$ for all $\theta$, the denominator of $\bar{F}_{D}\left(q^{*}(w, \theta)\right)$ in (14) is increasing in $\theta$, which implies $\bar{F}_{D}\left(q^{*}(w, \theta)\right)$ is decreasing and $q^{*}(w, \theta)$ is monotonically increasing in $\theta$ on $[\underline{\theta}, \bar{\theta}]$, thus the seller can differentiate among the buyer types, and the contract price schedule is volume dependent. By (A.7), the optimal reservation 
price schedule is

$$
\begin{aligned}
R(w, \theta) & =\frac{1}{1+r_{B}} \varphi\left(q^{*}(w, \theta), w, \theta\right)-\hat{\pi}_{B}\left(q^{*}(w, \theta), w, \theta\right) \\
& =\frac{1}{1+r_{B}} \varphi\left(q^{*}(w, \theta), w, \theta\right)-\hat{\pi}_{B}\left(q^{*}(w, \underline{\theta}), w, \underline{\theta}\right)-\left.\int_{\underline{\theta}}^{\theta} \frac{d \hat{\pi}_{B}(q, w, a)}{d a}\right|_{q=q^{*}(w, a)} d a \\
& =\frac{1}{1+r_{B}}\left(\varphi\left(q^{*}(w, \theta), w, \theta\right)-\left.\int_{\underline{\theta}}^{\theta} \frac{\partial \varphi(q, w, a)}{\partial a}\right|_{q=q^{*}(w, a)} d a\right) .
\end{aligned}
$$

Finally, $R\left(q^{*}(w, \theta), w\right)$ can be calculated by combining the two maps $q^{*}(w, \theta)$ and $R(w, \theta)$ on $[\underline{\theta}, \bar{\theta}]$. This completes the proof.

Proof of Proposition 3: The steps of the solution to the seller's optimization problem follows a similar outline as that for Proposition 2. First, with the capacity constraints, again defining $V(q, w, \theta)=$ $\pi_{S}(q, w, \theta)-R(w, \theta)$, we can rewrite the seller's optimization problem as

$$
\begin{aligned}
\max _{R(\cdot)} & \int_{\underline{\theta}}^{\bar{\theta}}(R(w, \theta)+V(q(w, \theta), w, \theta)) d F_{\theta}(\theta) \\
\text { s.t. } & q(w, \theta)=\arg \max _{\xi} \hat{\pi}_{B}(\xi, w, \theta), \quad \forall \theta \in[\underline{\theta}, \bar{\theta}] \\
& \hat{\pi}_{B}(q(w, \theta), w, \theta) \geq 0, \quad \forall \theta \in[\underline{\theta}, \bar{\theta}] \\
& \int_{\underline{\theta}}^{\bar{\theta}}(R(w, \theta)+V(q(w, \theta), w, \theta)) d F_{\theta}(\theta) \geq 0, \\
& 0 \leq q(w, \theta) \leq K .
\end{aligned}
$$

First consider $\beta \geq E[s](1-\phi) /\left(1+r_{S}\right)$. In this case, in (A.27), relaxing the capacity constraint together with the seller's IR constraint, the solution proceeds exactly as given in the proof of Proposition 2, with the exception that at the final pointwise optimization step, we need to ensure $0 \leq q(w, \theta) \leq K$. But since $H(q, w, \theta)$ given in (A.11) is concave, we conclude

$$
q^{*}(w, \theta, K)=\min \left\{K, \bar{F}_{D}^{-1}\left(\frac{\left(1+r_{S}\right) \beta-E[s](1-\phi)-(\rho-1) G(w)}{\eta(w, \theta)-(\rho-1) G(w)}\right)\right\} .
$$

Note that $q^{*}(w, \theta, K)=K$ if

$$
\eta(w, \theta) \bar{F}_{D}(K) \geq\left(1+r_{S}\right) \beta-E[s](1-\phi)-(\rho-1) G(w) F_{D}(K)
$$

and $q^{*}(w, \theta, K)<K$ otherwise.

Next consider the case where $\beta<E[s](1-\phi) /\left(1+r_{S}\right)$. Note that in this case the seller's optimal production is $K$, since every unit unsold to the buyer at $t=1$ brings in positive expected net profit to the 
seller through spot sales. Therefore the seller's expected profit for buyer type $\theta$ is

$$
\begin{array}{r}
\pi_{S}(q, w, \theta)=R(w, \theta)+\left(\frac{E[s](1-\phi)}{1+r_{S}}-\beta\right) K+\frac{1}{1+r_{S}} \int_{w-\theta}^{\frac{w}{1-\phi}}(w-s(1-\phi)) d F_{s}(s) \cdot E_{D}[\min (D, q)] \\
+\frac{1}{1+r_{S}} \int_{\frac{w}{1-\phi}}^{\bar{s}}(w-s(1-\phi)) d F_{s}(s) \cdot q .
\end{array}
$$

In addition, the seller's IR constraint also changes, as now she can make profit by selling all her capacity at the spot with no contracting with the buyer. That is, her IR constraint becomes

$$
\int_{\underline{\theta}}^{\bar{\theta}}(R(w, \theta)+V(q(w, \theta), w, \theta)) d F_{\theta}(\theta) \geq K \cdot\left(\frac{E[s](1-\phi)}{1+r_{S}}-\beta\right) .
$$

Following similar steps to those given in the proof of Proposition 2 to plug in the buyer's IC constraints into the seller's objective function we obtain

$$
\pi_{S}(w) \equiv \int_{\underline{\theta}}^{\bar{\theta}} H(q, w, \theta)
$$

where

$$
\begin{aligned}
H(q, w, \theta)=(-\beta & \left.+\frac{E[s](1-\phi)}{1+r_{S}}\right) K+\frac{1}{1+r_{S}} \int_{w-\theta}^{\bar{s}}\left(\phi s+\theta-g_{\theta}(\theta)\right) d F_{s}(s) E_{D}[\min (D, q)] \\
+ & \frac{r_{S}-r_{B}}{\left(1+r_{S}\right)\left(1+r_{B}\right)}\left(\int_{w-\theta}^{\bar{s}}\left(s+\theta-g_{\theta}(\theta)-w\right) d F_{s}(s) E_{D}[\min (D, q)]\right. \\
& \left.+\int_{\frac{w}{1-\phi}}^{\bar{s}}(s(1-\phi)-w) d F_{s}(s) E_{D}\left[(q-D)^{+}\right]\right) .
\end{aligned}
$$

Again, temporarily relaxing the seller's IR constraint and the capacity constraint, we can maximize this objective function pointwise. The first order condition for a given $\theta$ is

$$
\begin{aligned}
\frac{d H(q, w, \theta)}{d q}=\frac{1}{1+r_{S}} \int_{w-\theta}^{\bar{s}}\left(\phi s+\theta-g_{\theta}(\theta)\right) d F_{s}(s) \bar{F}_{D}(q) \\
+\frac{r_{S}-r_{B}}{\left(1+r_{S}\right)\left(1+r_{B}\right)}\left(\int_{w-\theta}^{\bar{s}}\left(s+\theta-g_{\theta}(\theta)-w\right) d F_{s}(s) \bar{F}_{D}(q)\right. \\
\left.\quad+\int_{\frac{w}{1-\phi}}^{\bar{s}}(s(1-\phi)-w) d F_{s}(s) F_{D}(q)\right)=0 .
\end{aligned}
$$


The second derivative of $H(q, w, \theta)$ with respect to $q$ is

$$
\begin{aligned}
\frac{d^{2} H(q, w, \theta)}{d q^{2}}=-\frac{f_{D}(q)}{1+r_{S}} \int_{w-\theta}^{\bar{s}}\left(\phi s+\theta-g_{\theta}(\theta)\right) d F_{s}(s) & \\
& -\frac{\left(r_{S}-r_{B}\right) f_{D}(q)}{\left(1+r_{S}\right)\left(1+r_{B}\right)}\left(\int_{w-\theta}^{\bar{s}}\left(s+\theta-g_{\theta}(\theta)-w\right) d F_{s}(s)\right. \\
& \left.-\int_{\frac{w}{1-\phi}}^{\bar{s}}(s(1-\phi)-w) d F_{s}(s)\right) .
\end{aligned}
$$

Note that since $s(1-\phi)-w<0$ for $w-\theta \leq s \leq w /(1-\phi)$, we have

$$
\int_{\frac{w}{1-\phi}}^{\bar{s}}(s(1-\phi)-w) d F_{s}(s) \geq \int_{w-\theta}^{\bar{s}}(s(1-\phi)-w) d F_{s}(s)
$$

and thus

$$
\int_{w-\theta}^{\bar{s}}\left(s+\theta-g_{\theta}(\theta)-w\right) d F_{s}(s)-\int_{\frac{w}{1-\phi}}^{\bar{s}}(s(1-\phi)-w) d F_{s}(s) \leq \int_{w-\theta}^{\bar{s}}\left(\phi s+\theta-g_{\theta}(\theta)\right) d F_{s}(s) .
$$

Plugging (A.36) into (A.34), we then have $d^{2} H(q, w, \theta) / d q^{2} \leq 0$, i.e., the pointwise objective function is weakly concave. Therefore the solution to (A.33) for each $\theta$ maximizes the objective function pointwise. Further, since $q=0$ is feasible for each $\theta$ and yields an expected profit of $E[s](1-\phi)\left(1+r_{S}\right)^{-1}-\beta$, concavity of $H(q, w, \theta)$ implies that the solution to (A.33) also maximizes the seller's problem without relaxing her IR constraint. Now, solving (A.33), combining it with the capacity constraint $q(w, \theta) \leq K$ and concavity of $H(q, w, \theta)$ we obtain

$$
q^{*}(w, \theta, K)=\min \left\{K, \bar{F}_{D}^{-1}\left(\frac{-(\rho-1) G(w)}{\eta(w, \theta)-(\rho-1) G(w)}\right)\right\}
$$

Therefore, $q^{*}(w, \theta, K)=K$ if and only if

$$
-(\rho-1) G(w) \leq \bar{F}_{D}(K)(\eta(w, \theta)-(\rho-1) G(w)) .
$$

Combining (A.37) and (A.28) gives us the desired expression. Further, combining (A.38) with (A.29), we obtain the condition

$$
\eta(w, \theta) \bar{F}_{D}(K) \geq\left(\left(1+r_{S}\right) \beta-E[s](1-\phi)\right)^{+}-(\rho-1) G(w) F_{D}(K)
$$

for $q^{*}(w, \theta, K)$ to equal $K$. As we have shown in the proof of Proposition 2, $\eta(w, \theta)$ is increasing in $\theta$. Therefore, if $\eta(w, \underline{\theta}) \bar{F}_{D}(K) \geq\left(\left(1+r_{S}\right) \beta-E[s](1-\phi)\right)^{+}-(\rho-1) G(w) F_{D}(K)$, (A.39) is satisfied by all $\theta \in[\underline{\theta}, \bar{\theta}]$, i.e., $q^{*}(w, \theta, K)=K$ for all $\theta \in[\underline{\theta}, \bar{\theta}]$, and there is flat pricing. On the other hand, if $\eta(w, \bar{\theta}) \bar{F}_{D}(K) \leq\left(\left(1+r_{S}\right) \beta-E[s](1-\phi)\right)^{+}-(\rho-1) G(w) F_{D}(K), q^{*}(w, \theta, K) \leq K$, for all $\theta$. Since $\eta(w, \theta)$ is increasing in $\theta$ for all $\theta$, the denominator of $\bar{F}_{D}\left(q^{*}(w, \theta)\right)$ in (14) is increasing in $\theta$, which implies 
$\bar{F}_{D}\left(q^{*}(w, \theta, K)\right)$ is decreasing and $q^{*}(w, \theta, K)$ is increasing in $\theta$ on $[\underline{\theta}, \bar{\theta}]$. Otherwise, there will be a $\theta_{c} \in[\underline{\theta}, \bar{\theta}]$ such that for $\theta \leq \theta_{c}, q^{*}(w, \theta, K) \leq K$ and increasing in $\theta$, while for $\theta \geq \theta_{c}, q^{*}(w, \theta, K)=K$. This completes the proof.

Proof of Corollary 1: For the case $K>\bar{D}$ and $\beta>E[s](1-\phi)\left(1+r_{S}\right)^{-1}$, the results can be directly observed from the flat price conditions. For low $K$ and $\beta$ cases, we derive the results by exploring the effects of the parameters on the two sides of the inequality

$$
\eta(w, \theta) \bar{F}_{D}(K) \geq\left(\left(1+r_{S}\right) \beta-E[s](1-\phi)\right)^{+}-(\rho-1) F_{D}(K) G(w),
$$

which, by Proposition (3) defines the menu structure for $\theta=\underline{\theta}$ and $\theta=\bar{\theta}$. We will only show the result for $K$. The remaining results will follow similar fashion.

For a given $\theta$, by rearranging (A.40), we obtain

$$
(\eta(w, \theta)-(\rho-1) G(w)) \bar{F}_{D}(K)+(\rho-1) G(w) \geq\left(\left(1+r_{S}\right) \beta-E[s](1-\phi)\right)^{+} .
$$

Since, as we have shown in the proof of Proposition 2, $\eta(w, \theta)-(\rho-1) G(w) \geq 0$ for all $w$ and $\theta$, the left hand side of (A.41) decreases as $K$ increases. Therefore, when $K$ increases (A.41) is less likely to be satisfied for both $\theta=\underline{\theta}$ and $\theta=\bar{\theta}$, which, by parts (i)-(iii) of Proposition 3 means that the contract is less likely to be flat price, and more likely to be mixed menu or fully volume dependent.

Proof of Proposition 4: The proof proceeds by applying the chain rule twice with $\theta$ as the common differentiator for taking the second derivative of $R(w, \theta)$ with respect to $q(\theta)$. We then evaluate the sign of the obtained second derivative for the $w$ ranges given in parts (i) and (ii) to obtian conditions for concavity and convexity of $R$ with respect to $q$.

By Proposition 2, given that the flat price conditions are not satisfied, the optimal reservation fee, $R(w, \theta(q))$, will depend on $\theta$. Using the implicit function theorem, we then have

$$
\frac{d R(w, \theta(q))}{d q}=\frac{d R(w, \theta)}{d \theta}\left(\frac{d q^{*}(w, \theta)}{d \theta}\right)^{-1}
$$

and

$$
\frac{d^{2} R(w, \theta(q))}{d q^{2}}=\left(\frac{d^{2} R(w, \theta)}{d \theta^{2}} \frac{d q^{*}(w, \theta)}{d \theta}-\frac{d R(w, \theta)}{d \theta} \frac{d^{2} q^{*}(w, \theta)}{d \theta^{2}}\right) \cdot\left(\frac{d q^{*}(w, \theta)}{d \theta}\right)^{-3}
$$

where $q^{*}(w, \theta)$ is given by (A.28). First, differentiating (14) with respect to $\theta$, we have

$$
-f_{D}\left(q^{*}(w, \theta)\right) \frac{d q^{*}(w, \theta)}{d \theta} \varrho(w, \theta)+\bar{F}_{D}\left(q^{*}(w, \theta)\right) \frac{d \varrho(w, \theta)}{d \theta}=0 .
$$

for all $q^{*}(w, \theta)<\min (K, \bar{D})$. As we have shown in the proof of Proposition 2, $\varrho(w, \theta) \geq 0$, for all $(w, \theta)$. 
Further, differentiating (A.15)

$$
\frac{d \varrho(w, \theta)}{d \theta}=\left(1-\rho \frac{d g_{\theta}(\theta)}{d \theta}\right) \bar{F}_{s}(w-\theta)+f_{s}(w-\theta) \nu(w, \theta)
$$

where we define $\nu(w, \theta)$ in (A.17). In the proof of Proposition 2, we showed $\nu(w, \theta) \geq 0, \forall w \geq \underline{s}+\theta$. Furthermore, the condition $d g_{\theta}(\theta) / d \theta \leq 0$ guarantee that $d \varrho(w, \theta) / d \theta \geq 0$. Therefore, by (A.44) it follows that $d q^{*}(w, \theta) / d \theta \geq 0$. Now by (16),

$$
\frac{d R(w, \theta)}{d \theta}=\left.\left(1+r_{B}\right)^{-1} \frac{d \varphi(q, w, \theta)}{d q}\right|_{q=q^{*}(w, \theta)} q^{\prime}(w, \theta)
$$

and

$$
\begin{array}{r}
\frac{d^{2} R(w, \theta)}{d \theta^{2}}=\left(1+r_{B}\right)^{-1}\left(\left.\frac{d \varphi(q, w, \theta)}{d q}\right|_{q=q^{*}(w, \theta)} \cdot \frac{d^{2} q^{*}(w, \theta)}{d \theta^{2}}+\left.\frac{\partial \varphi(q, w, \theta)}{\partial q}\right|_{q=q^{*}(w, \theta)} \frac{d q^{*}(w, \theta)}{d \theta}\right. \\
\left.+\left.\frac{d^{2} \varphi(q, w, \theta)}{d q^{2}}\right|_{q=q^{*}(w, \theta)}\left(\frac{d q^{*}(w, \theta)}{d \theta}\right)^{2}\right)
\end{array}
$$

Plugging (A.44), (A.46) and (A.47) in (A.43), we obtain

$\frac{d^{2} R(w, \theta(q))}{d q^{2}}=\frac{\bar{F}_{D}\left(q^{*}(w, \theta)\right)}{\left(1+r_{B}\right) \frac{d q^{*}(w, \theta)}{d \theta}}\left(\bar{F}_{s}(w-\theta)-\frac{d \varrho(w, \theta)}{d \theta} \frac{1}{\varrho(w, \theta)}\left(E_{s}\left[(s+\theta-w)^{+}\right]-E_{s}\left[(s(1-\phi)-w)^{+}\right]\right)\right)$.

Now suppose $w \leq \underline{s}(1-\phi)$. Then by (A.45),

$$
\varrho(w, \theta)=\rho\left(\phi E[S]+\theta-g_{\theta}(\theta)\right)
$$

and

$$
\frac{d \varrho(w, \theta)}{d \theta}=\rho\left(1-\frac{d g_{\theta}(\theta)}{d \theta}\right) .
$$

Plugging (A.49) and (A.50) in (A.48), it then follows that

$$
\frac{d^{2} R(w, \theta(q))}{d q^{2}}=\frac{1}{\left(1+r_{B}\right) \frac{d q^{*}(w, \theta)}{d \theta}} \cdot \frac{-g_{\theta}(\theta)+\frac{d g_{\theta}(\theta)}{d \theta}(\phi E[s]+\theta)}{\phi E[s]+\theta-g_{\theta}(\theta)} \leq 0,
$$

which proves part $(i)$.

Now, consider any $w=\underline{s}+\tilde{\theta}$, where $\tilde{\theta} \in[\underline{\theta}, \bar{\theta}]$, and for any $\theta \geq \tilde{\theta}$, using the condition $\bar{s}(1-\phi) \leq \underline{s}+\underline{\theta}$ :

$$
\varrho(w, \theta)=\rho\left(\phi E[s]+\theta-g_{\theta}(\theta)\right)-(\rho-1) E\left[(s+\tilde{\theta}-s(1-\phi))^{+}\right],
$$


and $d \varrho(w, \theta) / d \theta$ again satisfies (A.50). Plugging (A.52) and (A.50) in (A.48), we then have

$$
\frac{d^{2} R(w, \theta(q))}{d q^{2}}=\frac{1}{\left(1+r_{B}\right) \frac{d q^{*}(w, \theta)}{d \theta}}\left(1-\frac{\rho\left(1-\frac{d g_{\theta}(\theta)}{d \theta}\right)(E[s]+\theta-\underline{s}-\tilde{\theta})}{\rho\left(\phi E[s]+\theta-g_{\theta}(\theta)\right)-(\rho-1) E\left[(s+\tilde{\theta}-s(1-\phi))^{+}\right]}\right) .
$$

Simplifying (A.53), $d^{2} R(w, \theta(q)) / d q^{2} \leq 0$ if and only if

$$
\rho \geq \frac{E\left[(s+\tilde{\theta}-s(1-\phi))^{+}\right]}{g_{\theta}(\theta)-\frac{d g_{\theta}(\theta)}{d \theta}(E[s]-\underline{s}+\theta-\tilde{\theta})+E\left[(s(1-\phi)-\underline{s}-\tilde{\theta})^{+}\right]} .
$$

This completes the proof.

Proof of Corollary 3: We will first show that if $\frac{d^{2} g_{\theta}}{d \theta^{2}} \geq 0$, the right hand side of (17) is non-decreasing for $\theta>\tilde{\theta}$. Combining this with the condition (17) itself will imply that we can find boundary $\rho$ values above and below which the reservation price curve as a function of quantity will be concave and convex for all $\theta>\tilde{\theta}$ respectively.

Taking the derivative of the denominator in the right hand side of (17) with respect to $\theta$, we obtain $-d^{2} g_{\theta}(\theta) /(d \theta)^{2}(E[s]-\underline{s}+\theta-\tilde{\theta})$, which is negative for $\theta \geq \tilde{\theta}$ given $\frac{d^{2} g_{\theta}}{d \theta^{2}} \geq 0$. Therefore the denominator is non-increasing and the right hand side of (17) is non-decreasing in $\theta$. Now notice that $g_{\theta}(\bar{\theta})=0$ and $d g_{\theta}(\bar{\theta}) / d \theta=-1$. Therefore,

$$
\frac{E\left[(s+\tilde{\theta}-s(1-\phi))^{+}\right]}{g_{\theta}(\theta)-\frac{d g_{\theta}(\theta)}{d \theta}(E[s]-\underline{s}+\theta-\tilde{\theta})+E\left[(s(1-\phi)-\underline{s}-\tilde{\theta})^{+}\right]} \leq \frac{E\left[(w-s(1-\phi))^{+}\right]}{E[s]+\bar{\theta}-w+E\left[(s(1-\phi)-w)^{+}\right]},
$$

for all $\theta \in[\tilde{\theta}, \bar{\theta}]$, where the right hand side of (A.55) is the right hand side of (17) evaluated at $\bar{\theta}$. Similarly, evaluating the right hand side of (17) at $\tilde{\theta}$, and since $d g_{\theta}(\theta) / d \theta \leq 0$ for all $\theta$, we obtain

$$
\frac{E\left[(s+\tilde{\theta}-s(1-\phi))^{+}\right]}{g_{\theta}(\tilde{\theta})-\frac{d g_{\theta}(\tilde{\theta})}{d \theta}(E[s]-\underline{s})+E\left[(s(1-\phi)-\underline{s}-\tilde{\theta})^{+}\right]} .
$$

Hence, again by (17) and its monotonicity, it follows that on the range $\theta \in[\tilde{\theta}, \bar{\theta}], R(w, \theta(q))$ is concave in $q$ if $\rho$ is larger than the right hand side of (A.55), and convex in $q$ if $\rho$ is smaller than (A.56).

Proof of Proposition 5: The proof will proceed by taking the total derivative of the optimal contract quantity for a given $\theta$ with respect to $w$. From the resulting expression, we will isolate $d q^{*}(w, \theta) / d w$, take the expectation over $[\underline{\theta}, \bar{\theta}]$ and evaluate its sign for the desired $w$ ranges.

First, applying $\bar{F}_{D}$ to both sides of (14) and subsequently taking the total derivatives with respect to $w$, we have

$$
-f_{D}\left(q^{*}(w, \theta)\right) \frac{d q^{*}(w, \theta)}{d w} \varrho(w, \theta)+\bar{F}_{D}\left(q^{*}(w, \theta)\right) \frac{d \varrho(w, \theta)}{d w}=(\rho-1) \bar{F}_{s}\left(\frac{w}{1-\phi}\right)
$$


Now, when $r_{S} \geq r_{B}$, the right hand side of (A.57) is positive. Further, as we have shown in the proof of Proposition 2, $\frac{d \varrho(w, \theta)}{d w} \leq 0$ for all $w \geq 0$. It then follows from (A.57) that $\frac{d q^{*}(w, \theta)}{d w} \leq 0$. Therefore when $r_{S} \geq r_{B}$, for all $w \geq 0$,

$$
\frac{d E_{\theta}\left[q^{*}(w, \theta)\right]}{d w}=\int_{\underline{\theta}}^{\bar{\theta}} \frac{d q^{*}(w, \theta)}{d w} d F_{\theta}(\theta) \leq 0 .
$$

This proves part $(i)$. For part $(i i)$, when $r_{S}<r_{B}$ and $w<\underline{s}+\theta$, by (A.16), $\frac{d \varrho(w, \theta)}{d w} \geq 0$. Substituting in (A.57) and noticing that the right hand side is negative, it follows that $\frac{d q^{*}(w, \theta)}{d w} \geq 0$ for $w<\underline{s}+\theta$ for all $t$. It then follows that for $w<\underline{s}+\underline{\theta}, \frac{d E_{\theta}\left[q^{*}(w, \theta)\right]}{d w}=\int_{\underline{\theta}}^{\bar{\theta}} \frac{d q^{*}(w, \theta)}{d w} d F_{\theta}(\theta) \geq 0$. However, when $w>\underline{s}+\theta$, as $\rho \rightarrow 1$, the right hand side of (A.57) vanishes, and since $\frac{d \varrho(w, \theta)}{d w} \leq 0, \frac{d q^{*}(w, \theta)}{d w} \leq 0$ follows. On the other hand, when $q^{*}(w, \theta)$ is sufficiently close to $\bar{D}$, the second term on the left hand side of (A.57) vanishes, which implies $\frac{d q^{*}(w, \theta)}{d w} \geq 0$. This completes the proof.

Proof of Proposition 6: The sketch of the proof is as follows: First, using the results of Proposition 2, we calculate $\pi_{S}^{*}(w)$. Then taking the total derivative of $\pi_{S}^{*}(w)$ with respect to $w$, we calculate $d \pi_{S}^{*}(w) / d w$. Using this expression, we derive the shape of the curve $\pi_{S}^{*}(w)$. More specifically, we show that, $\pi_{S}$ is strictly monotonically decreasing in $w$ when $r_{S}>r_{B}$; flat until $w=\underline{s}+\underline{\theta}$ and monotonically decreasing afterwards when $r_{S}=r_{B}$; and peaks on $[\underline{s}+\underline{\theta}, \underline{s}+\bar{\theta}]$ when $r_{S}<r_{B}$.

First, substituting $q^{*}(w, \theta)$ in the seller's expected profit function, we have

$$
\pi_{S}^{*}(w)=\int_{\underline{\theta}}^{\bar{\theta}} H\left(q^{*}(w, \theta), w, \theta\right) d F_{\theta}(\theta),
$$

where $H(q, w, \theta)$ is the integrand in (A.10). Taking the total derivative with respect to $w$ in (A.59), and applying the envelope theorem, we have

$$
\begin{aligned}
\frac{d \pi_{S}^{*}(w)}{d w}= & \int_{\underline{\theta}}^{\bar{\theta}}\left(\frac{\partial H\left(q^{*}(w, \theta), w, \theta\right)}{\partial w}+\left.\frac{\partial H\left(q^{*}(w, \theta), w, \theta\right)}{\partial q}\right|_{q=q^{*}(w, \theta)} \cdot \frac{d q^{*}(w, \theta)}{d w}\right) d F_{\theta}(\theta) \\
= & \int_{\underline{\theta}}^{\bar{\theta}} \frac{\partial H\left(q^{*}(w, \theta), w, \theta\right)}{\partial w} d F_{\theta}(\theta) \\
= & \int_{\underline{\theta}}^{\bar{\theta}}\left\{\begin{array}{l}
-(\rho-1) \bar{F}_{s}\left(\frac{w}{1-\phi}\right) q^{*}(w, \theta)-(\rho-1)\left(F_{s}\left(\frac{w}{1-\phi}\right)-F_{s}(w-\theta)\right) E_{D}\left[\min \left(D, q^{*}(w, \theta)\right)\right] \\
\quad \\
\quad-(\rho-1) g_{\theta}(\theta) f_{s}(w-\theta) E_{D}\left[\min \left(D, q^{*}(w, \theta)\right)\right] \\
\left.\quad-f_{s}(w-\theta)\left(\phi(w-\theta)+\theta-g_{\theta}(\theta)\right) E_{D}\left[\min \left(D, q^{*}(w, \theta)\right)\right]\right\} d F_{\theta}(\theta)
\end{array}\right.
\end{aligned}
$$


By (A.17), for any given $\theta \in[\underline{\theta}, \bar{\theta}]$,

$$
\frac{\partial H\left(q^{*}(w, \theta), w, \theta\right)}{\partial w}=\left\{\begin{array}{cl}
-(\rho-1)\left(q^{*}(w, \theta)-F_{s}\left(\frac{w}{1-\phi}\right) E_{D}\left[\left(q^{*}(w, \theta)-D\right)^{+}\right]\right) & \text {for } w<\underline{s}+\theta ; \\
-f_{s}(w-\theta) \nu(w, \theta) E_{D}\left[\min \left(D, q^{*}(w, \theta)\right)\right] & \\
-(\rho-1) \bar{F}_{s}\left(\frac{w}{1-\phi}\right) E_{D}\left[\left(q^{*}(w, \theta)-D\right)^{+}\right] & \text {for } w \in[\underline{s}+\theta, \bar{s}+\theta] ; \\
0 & \text { for } w>\bar{s}+\theta .
\end{array}\right.
$$

Substituting (A.61) in (A.60), we then have

$$
\frac{d \pi_{S}^{*}(w)}{d w}=-(\rho-1) \int_{\underline{\theta}}^{\bar{\theta}}\left(q^{*}(w, \theta)-F_{s}\left(\frac{w}{1-\phi}\right) E_{D}\left[\left(q^{*}(w, \theta)-D\right)^{+}\right]\right) d F_{\theta}(\theta)
$$

for $w<\underline{s}+\underline{\theta}$, and

$$
\begin{array}{r}
\frac{d \pi_{S}^{*}(w)}{d w}=-\int_{\underline{\theta}}^{\tilde{\theta}} f_{s}(w-\theta) \nu(w, \theta) E_{D}\left[\min \left(D, q^{*}(w, \theta)\right)\right]+(\rho-1) \bar{F}_{s}\left(\frac{w}{1-\phi}\right) E_{D}\left[\left(q^{*}(w, \theta)-D\right)^{+}\right] d F_{\theta}(\theta) \\
\left.-(\rho-1) \int_{\tilde{\theta}}^{\bar{\theta}}\left(q^{*}(w, \theta)-F_{s}\left(\frac{w}{1-\phi}\right) E_{D}\left[\left(q^{*}(w, \theta)-D\right)^{+}\right]\right) d F_{\theta}(\theta), \quad \text { (A. } 63\right)
\end{array}
$$

for any $w=\underline{s}+\tilde{\theta}$, where $\tilde{\theta} \in[\underline{\theta}, \bar{\theta}]$. Further, as shown in the proof of Proposition 2 that $\nu(w, \theta) \geq 0, \forall w \geq$ $\underline{s}+\theta$; and by (A.61), $d \pi_{S}^{*}(w) / d w \leq 0$ for all $w \geq \underline{s}+\bar{\theta}$. Now, when $\rho>1$, since $F_{s}\left(\frac{w}{1-\phi}\right) E_{D}\left[\left(q^{*}(w, \theta)-D\right)^{+}\right]<$ $q^{*}(w, \theta)$ for all $\theta$, the integrand in (A.62) is positive for all $\theta$. As a consequence, $d \pi_{S}^{*}(w) / d w<0$ for $0 \leq w<\underline{s}+\underline{\theta}$. Similarly, since both terms in (A.63) are negative, $d \pi_{S}^{*}(w) / d w<0$ also follows for $\underline{s}+\underline{\theta} \leq w \leq \bar{s}+\bar{\theta}$. Therefore, for $\rho>1, \pi_{S}^{*}(w)$ is maximized at $w^{*}=0$, which proves part $(i)$. When $\rho=1$, by $(\mathrm{A} .62), d \pi_{S}^{*}(w) / d w=0$ for $0 \leq w<\underline{s}+\underline{\theta}$, and by $(\mathrm{A} .63), d \pi_{S}^{*}(w) / d w<0$ for $\underline{s}+\underline{\theta} \leq w \leq \bar{s}+\bar{\theta}$. Hence $\pi_{S}^{*}(w)$ is flat on $w \in[0, \underline{s}+\underline{\theta}]$, strictly decreasing on $[\underline{s}+\underline{\theta}, \bar{s}+\bar{\theta}]$, and flat again for $w>\bar{s}+\bar{\theta}$, and thus is maximized for any $w \in[0, \underline{s}+\underline{\theta}]$, as stated in part (ii). Finally, when $\rho<1$, by (A.62), $d \pi_{S}^{*}(w) / d w>0$ for $0 \leq w<\underline{s}+\underline{\theta}$. Further, by (A.63), $d \pi_{S}^{*}(w) / d w>0$ for $w=\underline{s}+\underline{\theta}$, and $\pi_{S}^{*}(w)$ is non-increasing for $w>\bar{s}+\bar{\theta}$. Since $\pi_{S}^{*}(w)$ is continuous in $w$, it follows that for $\rho<1, \pi_{S}^{*}(w)$ is maximized at a $w^{*} \in[\underline{s}+\underline{\theta}, \bar{s}+\bar{\theta}]$ as stated in part $($ iii). This completes the proof.

Proof of Proposition 7: The steps of the proof proceed similar to the proof of Proposition 6, this time using the contract price and quantity expressions from Proposition 3, highlighting the differences in the shape of the $\pi_{S}$ curve as a function of $w$ as given in the proposition statement.

First, writing $\pi_{S}$ as a function of $w$, for $q^{*}(w, \theta)<K$, i.e., $\theta \leq \theta^{c}(w)$, the price schedule is volume dependent, i.e., $q^{*}(w, \theta)$ is increasing in $\theta$. On the other hand, for $\theta \geq \theta^{c}(w), q^{*}(w, \theta)=K$, the price schedule is flat, and the seller's expected profit from any type $\theta$ buyer in this region is the sum of the reservation price she will obtain for contracting $K$ units, $R\left(w, \theta^{c}(w)\right)$, and the profit she will make with $K$ 
contracts sold to type $\theta$ buyer, $V(K, w, \theta)$. Therefore, we have

$$
\pi_{S}(w)=\int_{\underline{\theta}}^{\theta^{c}(w)} H\left(q^{*}(w, \theta), w, \theta\right) d F_{\theta}(\theta)+\bar{F}_{\theta}\left(\theta^{c}(w)\right) R\left(w, \theta^{c}(w)\right)+\int_{\theta^{c}(w)}^{\bar{\theta}} V(K, w, \theta) d F_{\theta}(\theta),
$$

or equivalently,

$$
\pi_{S}(w)=\int_{\underline{\theta}}^{\bar{\theta}} \pi_{S}(w, \theta) d F_{\theta}(\theta)
$$

where

$$
\pi_{S}(w, \theta)= \begin{cases}H\left(q^{*}(w, \theta), w, \theta\right) & \text { for } \theta<\theta^{c}(w) \\ R\left(w, \theta^{c}(w)\right)+V(K, w, \theta) & \text { for } \theta \geq \theta^{c}(w) .\end{cases}
$$

Now,

$$
\frac{d \pi_{S}(w)}{d w}=\int_{\underline{\theta}}^{\bar{\theta}} \frac{d \pi_{S}(w, \theta)}{d w} d F_{\theta}(\theta) .
$$

For $\theta<\theta^{c}(w)$, by the envelope theorem, $d \pi_{S}(w, \theta) / d w=\partial H\left(q^{*}(w, \theta), w, \theta\right) / \partial w$, and hence

$$
\frac{\partial H\left(q^{*}(w, \theta), w, \theta\right)}{\partial w}=\left\{\begin{aligned}
-\frac{r_{S}-r_{B}}{\left(1+r_{S}\right)\left(1+r_{B}\right)}\left(E_{D}\left[\min \left(D, q^{*}(w, \theta)\right)\right]\right. & \\
\left.+\bar{F}_{s}\left(\frac{w}{1-\phi}\right) E_{D}\left[\left(q^{*}(w, \theta)-D\right)^{+}\right]\right) & \text {for } w<\underline{s}+\theta ; \\
-\frac{1}{1+r_{S}} f_{s}(w-\theta) E_{D}\left[\min \left(D, q^{*}(w, \theta)\right)\right] \nu(w, \theta) & \text { for } w \in[\underline{s}+\theta, \bar{s}+\theta] ; \\
0 & \text { otherwise. }
\end{aligned}\right.
$$

where $\nu(w, \theta)=\phi(w-\theta)+\theta-g_{\theta}(\theta)+(\rho-1)\left(g_{s}(w-\theta)-g_{\theta}(\theta)\right)$. On the other hand, for $\theta \geq \theta^{c}(w)$, by taking the total derivative of $\pi_{S}(w, \theta)$ with respect to $w$,

$$
\frac{d \pi_{S}(w, \theta)}{d w}=\frac{\partial R\left(w, \theta^{c}(w)\right)}{\partial w}+\frac{\partial V(K, w, \theta)}{\partial w}+\frac{\partial R\left(w, \theta^{c}(w)\right)}{\partial \theta^{c}(w)} \frac{d \theta^{c}(w)}{d w} .
$$

Now, by (A.26),

$$
R\left(w, \theta^{c}(w)\right)=\frac{1}{1+r_{B}}\left(\varphi\left(K, w, \theta^{c}(w)\right)-\left.\int_{\underline{\theta}}^{\theta^{c}(w)} \frac{\partial \varphi(q, w, a)}{\partial a}\right|_{q=q^{*}(w, a)} d a\right) .
$$

Thus,

$$
\frac{\partial R\left(w, \theta^{c}(w)\right)}{\partial \theta^{c}(w)}=\frac{\partial \varphi\left(K, w, \theta^{c}(w)\right)}{\partial \theta^{c}(w)}-\frac{\partial \varphi\left(K, w, \theta^{c}(w)\right)}{\partial \theta^{c}(w)}=0,
$$

and

$$
\begin{aligned}
\frac{\partial R\left(w, \theta^{c}(w)\right)}{\partial w}= & \frac{\partial \varphi\left(K, w, \theta^{c}(w)\right)}{\partial w}-\int_{\underline{\theta}}^{\theta^{c}(w)} \frac{\partial^{2} \varphi(q, w, a)}{\partial w \partial a} d a \\
= & \frac{1}{1+r_{B}}\left\{-\bar{F}_{s}\left(w-\theta^{c}(w)\right) E_{D}[\min (D, K)]-\bar{F}_{s}\left(\frac{w}{1-\phi}\right) E_{D}\left[(K-D)^{+}\right]\right. \\
& \left.\quad+\int_{\underline{\theta}}^{\theta^{c}(w)} f_{s}(w-\theta) E_{D}[\min (D, K)] d \theta\right\} .
\end{aligned}
$$


In addition,

$\frac{\partial V(K, w, \theta)}{\partial w}=\frac{1}{1+r_{S}}\left(\bar{F}_{s}\left(\frac{w}{1-\phi}\right) E_{D}\left[(K-D)^{+}\right]-f_{s}(w-\theta)\left(\theta+\phi(w-\theta)-g_{s}(w-\theta)\right) E_{D}[\min (D, K)]\right)$.

Therefore, plugging (A.71)-(A.73) into (A.70), for $\theta \geq \theta^{c}(w)$ we then have

$$
\begin{aligned}
\frac{d \pi_{S}(w, \theta)}{d w}= & \frac{\partial R\left(w, \theta^{c}(w)\right)}{\partial w}+\frac{\partial V(K, w, \theta)}{\partial w} \\
= & -\frac{1}{1+r_{B}} \bar{F}_{s}(w-\underline{\theta}) E_{D}[\min (D, K)]-\frac{1}{1+r_{S}} f_{s}(w-\theta)\left(\theta+\phi(w-\theta)-g_{s}(w-\theta)\right) E_{D}[\min (D, K)] \\
& -\frac{r_{S}-r_{B}}{\left(1+r_{B}\right)\left(1+r_{S}\right)} \bar{F}_{s}\left(\frac{w}{1-\phi}\right) E_{D}\left[(K-D)^{+}\right]
\end{aligned}
$$

Simplifying (A.74), we obtain

$$
\frac{d \pi_{S}(w, \theta)}{d w}=\left\{\begin{array}{cl}
-\frac{1}{1+r_{B}} \bar{F}_{s}(w-\underline{\theta}) E_{D}[\min (D, K)]-\frac{r_{S}-r_{B}}{\left(1+r_{B}\right)\left(1+r_{S}\right)} \bar{F}_{s}\left(\frac{w}{1-\phi}\right) E_{D}\left[(K-D)^{+}\right] & \text {for } w<\underline{s}+\theta ; \\
-\frac{1}{1+r_{B}} \bar{F}_{s}(w-\underline{\theta}) E_{D}[\min (D, K)]-\frac{r_{S}-r_{B}}{\left(1+r_{B}\right)\left(1+r_{S}\right)} \bar{F}_{s}\left(\frac{w}{1-\phi}\right) E_{D}\left[(K-D)^{+}\right] & \\
-\frac{1}{1+r_{S}} f_{s}(w-\theta)\left(\theta+\phi(w-\theta)-g_{s}(w-\theta)\right) E_{D}[\min (D, K)] & \text { for } w \in[\underline{s}+\theta, \bar{s}+\theta] \\
0 & \text { otherwise }
\end{array}\right.
$$

for $\theta>\theta^{c}(w)$. By (A.68) and (A.75), $d \pi_{S}(w, \theta) / d w \leq 0$ for all $w>\underline{s}+\bar{\theta}$. Therefore, $\pi_{S}(w, \theta)$ attains its optimum on $w \in[0, \underline{s}+\bar{\theta}]$. Suppose $r_{B} \leq r_{S}$. Then, again by (A.68) and (A.75), $d \pi_{S}(w, \theta) / d w \leq 0$ for all $w<\underline{s}+\bar{\theta}$ as well. Hence, for $r_{B} \leq r_{S}, w^{*}=0$, i.e., selling the intermediate good is optimal for the supplier. This proves part (i).

Now suppose $r_{B}>r_{S}$. For part (ii)(a), suppose $E[s]+\bar{\theta}-g_{\theta}(\bar{\theta})>\beta\left(1+r_{S}\right)$. If $E[s](1-\phi)<\left(1+r_{S}\right) \beta$, then $\phi E[s]+\bar{\theta}-g_{\theta}(\bar{\theta})>\left(\beta\left(1+r_{S}\right)-E[s](1-\phi)\right)^{+}$, which is also automatically true when $E[s](1-\phi) \geq$ $\left(1+r_{S}\right) \beta$. This means that for small enough $\rho-1$ and $K, \eta(0, \bar{\theta}) \bar{F}_{D}(K)>\left(\beta\left(1+r_{S}\right)-E[s](1-\phi)\right)^{+}-$ $(\rho-1) F_{D}(K) G(0)$, i.e., in the optimal contract for $w=0, \theta^{c}(0)<\bar{\theta}$. Further, note that for $w<\underline{s}+\underline{\theta}$, we have

$$
\frac{d \pi_{S}(w, \theta)}{d w}=\left\{\begin{array}{cc}
-\frac{r_{S}-r_{B}}{\left(1+r_{S}\right)\left(1+r_{B}\right)}\left(E_{D}\left[\min \left(D, q^{*}(w, \theta)\right)\right]\right. & \text { for } \theta<\theta^{c}(w) \\
\left.+\bar{F}_{s}\left(\frac{w}{1-\phi}\right) E_{D}\left[\left(q^{*}(w, \theta)-D\right)^{+}\right]\right) & \text {for } \theta \geq \theta^{c}(w) \\
-\frac{1}{1+r_{B}} \bar{F}_{s}(w-\underline{\theta}) E_{D}[\min (D, K)]-\frac{r_{S}-r_{B}}{\left(1+r_{B}\right)\left(1+r_{S}\right)} \bar{F}_{s}\left(\frac{w}{1-\phi}\right) E_{D}\left[(K-D)^{+}\right]
\end{array}\right.
$$

and for $w \in[\underline{s}+\underline{\theta}, \underline{s}+\bar{\theta}], d \pi_{S}(w, \theta) / d w$ is proportional to $r_{B}-r_{S}$. As a result, for small enough $\rho-1$ and $K, \pi_{S}(w, \theta)$ is strictly decreasing at $w=0$, and never reaches the value attained at $w=0$ on $(\underline{s}+\underline{\theta}, \underline{s}+\bar{\theta}]$. That is $w^{*}=0$, proving part (ii)(a). Finally, for part (ii)(b), for any $\rho<1$, notice that for large enough $K$,

$$
\eta(w, \bar{\theta}) \bar{F}_{D}(K)<\left(\beta\left(1+r_{S}\right)-E[s](1-\phi)\right)^{+}-(\rho-1) F_{D}(K) G(w)
$$


for all $w \geq 0$, i.e., the optimal contract is fully volume dependent for all $w \geq 0$. In this case $d \pi_{S}(w, \theta) / d w=$ $\partial H\left(q^{*}(w, \theta), w, \theta\right) / \partial w$, and hence, as we have shown in the proof of Proposition $6, \pi_{S}(w, \theta)$ attains its maximum on $w \in(\underline{s}+\underline{\theta}, \underline{s}+\bar{\theta}]$. This completes the proof.

Proof of Proposition 8: The sketch of the proof is as follows: First, we obtain the total derivative of the supplier's profit $\pi_{S}$ with respect to $w$. Utilizing the fact that at $\rho=1, w^{*}=\underline{s}+\underline{\theta}$, plugging in the optimal contract quantity, $q^{*}(w, \theta)$ from (14), and having a Taylor expansion on the total derivative of $\pi_{S}$ with respect to $w$ at $\underline{s}+\underline{\theta}$, we obtain a Taylor expansion for $w^{*}$ in powers of $\rho-1$. We can then use this expansion to explore the effects of various parameters on $w^{*}$ for small $\rho-1$.

Given that $r_{S} \leq r_{B}$, by Proposition 6 , we know that $\underline{s}+\underline{\theta} \leq w^{*} \leq \underline{s}+\bar{\theta}$. Defining $\tilde{\theta}(w) \triangleq w-\underline{s}$, at optimality and by (A.63)

$$
\begin{aligned}
\left.\frac{d \pi_{S}^{*}(w)}{d w}\right|_{w=w^{*}}= & -\int_{\underline{\theta}}^{\tilde{\theta}\left(w^{*}\right)} f_{s}(w-\theta) \nu(w, \theta) E_{D}\left[\min \left(D, q^{*}(w, \theta)\right)\right] d F_{\theta}(\theta) \\
& -(\rho-1) \int_{\tilde{\theta}\left(w^{*}\right)}^{\bar{\theta}} E_{D}\left[\min \left(D, q^{*}(w, \theta)\right)\right] d F_{\theta}(\theta) \\
= & 0
\end{aligned}
$$

where $\nu(w, \theta)$ is defined in (A.17), and $q^{*}(w, \theta)$ is defined in (14). Define $\hat{q}(\theta)$ as the optimal reservation level for a type $\theta$ buyer when exercise price is $\underline{s}+\underline{\theta}$. By (14), we have

$$
\hat{q}(\theta)=\left.q^{*}(w, \theta)\right|_{w=\underline{s}+\underline{\theta}}=\bar{F}_{D}^{-1}\left(\frac{\left(1+r_{S}\right) \beta-E[s](1-\phi)}{\phi E[s]+\theta-g_{\theta}(\theta)}\right) .
$$

By applying the implicit function theorem to (A.78), combining with (A.79) and simplifying, it then follows that, for $\rho<1$,

$$
w^{*}=\underline{s}+\underline{\theta}-(\rho-1) \frac{\int_{\underline{\theta}}^{\bar{\theta}} E_{D}[\min (D, \hat{q}(\theta))] d F_{\theta}(\theta)}{f_{\theta}(\underline{\theta}) f_{s}(\underline{s})\left[\phi \underline{s}+\underline{\theta}-g_{\theta}(\underline{\theta})\right] E_{D}[\min (D, \hat{q}(\theta))]}+O\left((\rho-1)^{2}\right) .
$$

By (A.79) and (A.80), as $\operatorname{Var}[s]$ increases with a mean preserving spread that decreases $\underline{s}, w^{*}$ decreases for small enough $\rho-1$ values. Similarly, when there is a rightward shift for $\theta$ or $s$ distributions, $\underline{s}$ and $\underline{\theta}$ increase and $w^{*}$ increases when $\rho$ is close enough to 1 . The remaining comparative statics can be obtained similarly.

Proof of Proposition 9: For both parts (i) and (ii), the proof proceeds by plugging in the corresponding $w^{*}$ into (14), taking the expectation over $\theta$. For applying Taylor expansion around $\rho=1$. The effects of parameters can then be driven (for small enough $\rho-1$ for part (ii)) by using the resulting expression.

To see part $(i)$, first we know that when $r_{S}>r_{B}$, by Proposition 6 that $w^{*}=0$. Plugging in (14), and 
taking expectations over $\theta$, we have

$$
E_{\theta}\left[q^{*}(0, \theta)\right]=\int_{\underline{\theta}}^{\bar{\theta}} \bar{F}_{D}^{-1}\left(\frac{\left(1+r_{S}\right) \beta-\rho E[s](1-\phi)}{\rho\left(\phi E[s]+\theta-g_{\theta}(\theta)\right)}\right) d F_{\theta}(\theta)
$$

By (A.81), when there is a rightward shift in $s$, the numerator of the argument of $\bar{F}_{D}^{-1}$ decreases, and the denominator increases. Hence, the argument decreases and since $\bar{F}_{D}$ is monotonically decreasing, the integrand and consequently $E_{\theta}\left[q^{*}(0, \theta)\right]$ increases. The remaining comparative statics follow similarly. This completes the proof of part $(i)$.

For part (ii), plugging (A.80) in (14) and (15), and simplifying,

$$
E_{\theta}\left[q^{*}\left(w^{*}, \theta\right)\right]=\int_{\underline{\theta}}^{\bar{\theta}} \hat{q}(\theta) d F_{\theta}(\theta)+(\rho-1) \int_{\underline{\theta}}^{\bar{\theta}} \frac{\bar{F}_{D}(\hat{q}(\theta))}{f_{D}(\hat{q}(\theta))} \frac{E[s]-\underline{s}-\underline{\theta}+\theta-g_{\theta}(\theta)}{\phi E[s]+\theta-g_{\theta}(\theta)} d F_{\theta}(\theta)+O\left((\rho-1)^{2}\right) .
$$

By (A.79) and (A.82), we can observe the effects of the parameters on $E_{\theta}\left[q^{*}\left(w^{*}, \theta\right)\right]$. When there is a rightward shift in $s$, the numerator of the argument of $\bar{F}_{D}$ in (A.79) decreases, and the denominator in the same expression increases. Hence, the argument decreases and since $\bar{F}_{D}$ is monotonically decreasing, the integrand, $\hat{q}(\theta)$, increases in the constant term in (A.82). Similarly, with a $\theta$ rightward shift in $\theta$, the denominator in (A.79) increases, and again the constant term in (A.82) increases. Consequently $E_{\theta}\left[q^{*}\left(w^{*}, \theta\right)\right]$ increases with a rightward shift in $s$ or $\theta$ for $\rho$ sufficiently close to 1 . The remaining comparative statics follow similarly.

Proof of Proposition 10: The sketch of the proof is similar to those of Propositions 8 and 9. Specifically, for both parts (i) and (ii), the expressions for $w^{*}$ and $q^{*}(w, \theta)$ are plugged in the expression for $R\left(q^{*}(w, \theta), w^{*}\right)$. Then the expectation of $R\left(q^{*}(w, \theta), w^{*}\right)$ is taken over $\theta$ and Taylor expansion around $\rho=1$ is obtained. The effects of the parameters on the expected reservation fee are then derived from the final expression.

For part $(i)$, by Proposition 6 , since $r_{S}>r_{B}, w^{*}=0$. Plugging $w^{*}=0$ in (14) and (A.26) and taking the expectation over $\theta$,

$$
\begin{array}{r}
E_{\theta}\left[R\left(q^{*}(0, \theta), 0\right)\right]=\frac{1}{1+r_{B}} \int_{\underline{\theta}}^{\bar{\theta}}\left\{\left(E[s]+\theta-g_{\theta}(\theta)\right) E_{D}\left[\min \left(D, \bar{F}_{D}^{-1}\left(\frac{\left(1+r_{S}\right) \beta-\rho E[s](1-\phi)}{\rho\left(\phi E[s]+\theta-g_{\theta}(\theta)\right)}\right)\right)\right]\right. \\
\left.+E[s](1-\phi) E_{D}\left[\left(\bar{F}_{D}^{-1}\left(\frac{\left(1+r_{S}\right) \beta-\rho E[s](1-\phi)}{\rho\left(\phi E[s]+\theta-g_{\theta}(\theta)\right)}\right)-D\right)^{+}\right]\right\} d F_{\theta}(\theta) . \quad(\mathrm{A} .
\end{array}
$$

By (A.83), as $\rho$ increases, the numerator of the argument of $\bar{F}_{D}$ decreases and the denominator increases. Hence, the argument decreases, and the integrand increases. Therefore, $E_{\theta}\left[R\left(q^{*}(0, \theta), 0\right)\right]$ increases with $\rho$. The remaining comparative statics follow in a similar fashion. 
To see part (ii), plugging (A.80) in (A.26), taking expectations and simplifying, we obtain

$$
\begin{aligned}
E_{\theta}\left[R\left(q^{*}\left(w^{*}, \theta\right), w^{*}\right)\right]= & \int_{\underline{\theta}}^{\bar{\theta}}\left(E[s]+\theta-g_{\theta}(\theta)-\underline{s}-\underline{\theta}\right) E_{D}[\min (D, \hat{q}(\theta))] d F_{\theta}(\theta) \\
& +(\rho-1)\left(\int_{\underline{\theta}}^{\bar{\theta}}\left(\kappa+E[s]+\theta-g_{\theta}(\theta)-\underline{s}-\underline{\theta}\right) E_{D}[\min (D, \hat{q}(\theta))] d F_{\theta}(\theta)\right. \\
& \left.+\int_{\underline{\theta}}^{\bar{\theta}} \frac{\bar{F}_{D}(\hat{q}(\theta))^{2}\left[E[s]+\theta-g_{\theta}(\theta)-\underline{s}-\underline{\theta}\right]^{2}}{f_{D}(\hat{q}(\theta))\left[\phi E[s]+\theta-g_{\theta}(\theta)\right]} d F_{\theta}(\theta)\right)+O\left((\rho-1)^{2}\right)
\end{aligned}
$$

where $\hat{q}(\theta)$ is as defined in (A.79), and

$$
\kappa=\frac{\int_{\underline{\theta}}^{\bar{\theta}} E_{D}[\min (D, \hat{q}(\theta))] d F_{\theta}(\theta)}{f_{\theta}(\underline{\theta}) f_{s}(\underline{s})\left[\phi \underline{s}+\underline{\theta}-g_{\theta}(\underline{\theta})\right] E_{D}[\min (D, \hat{q}(\underline{\theta}))]} \geq 0 .
$$

Now,

$$
\int_{\underline{\theta}}^{\bar{\theta}}\left(E[s]+\theta-g_{\theta}(\theta)-\underline{s}-\underline{\theta}\right) d F_{\theta}(\theta)=E[s]-\underline{s} \geq 0 .
$$

Note that the integrand in (A.86) and also $E_{D}[\min (D, \hat{q}(\theta))]$ are both non-decreasing in $\theta$. Further, $E_{D}[\min (D, \hat{q}(\theta))] \geq 0$ implies that

$$
\int_{\underline{\theta}}^{\bar{\theta}}\left(E[s]+\theta-g_{\theta}(\theta)-\underline{s}-\underline{\theta}\right) E_{D}[\min (D, \hat{q}(\theta))] d F_{\theta}(\theta) \geq 0 .
$$

Therefore, the coefficient of $\rho-1$ in (A.84) is non-negative, which implies $E_{\theta}\left[R\left(q^{*}\left(w^{*}, \theta\right), w^{*}\right)\right]$ increases with $\rho$ when $\rho$ is sufficiently close to 1 . The remaining comparative statics can likewise be obtained. This completes the proof.

Proof of Proposition 11: The proof will proceed as the optimization of (19), with respect to the integrated supply chain production quantity $y(\theta)$. First, notice that (19) is strictly concave in $y(\theta)$. Hence, by taking the first order condition in (19) for each $\theta$-type, equating to zero and solving for $y$, the corresponding optimal production quantity for the supply chain is given as in (21). Substituting (21) into (19), and taking expectations over $\theta$, the first-best profit level for the centralized system is

$$
\begin{aligned}
\pi_{S C}=-\beta E_{\theta}\left[y^{*}(\theta)\right]+\frac{1}{1+r_{S C}} \int_{\underline{\theta}}^{\bar{\theta}}\left(p E[D]+E[s](1-\phi) E_{D}\left[\left(y^{*}(\theta)-D\right)^{+}\right]\right. \\
\left.-(E[s]+\theta) E_{D}\left[\left(D-y^{*}(\theta)\right)^{+}\right]\right) d F_{\theta}(\theta) \\
=\frac{1}{1+r_{S C}}\left((p-E[s]-E[\theta]) E[D]+\int_{\underline{\theta}}^{\bar{\theta}}(\phi E[s]+\theta) \int_{0}^{y^{*}(\theta)} x d F_{D}(x) d F_{\theta}(\theta)\right),
\end{aligned}
$$

as stated. 


\section{B The Analysis of the Fixed Buyer Type Case with Correlation Be- tween Demand and Spot Price}

In this section, we study the correlation between demand and spot price random variables for the fixed buyer type case that we presented in Section 4.

Let $F_{D, s}$, and $f_{D, s}$ denote the c.d.f. and p.d.f. of the joint distribution of $D$ and $s$ respectively, and $F_{D}(\cdot \mid s)$ denote the conditional distribution of $D$ given $s$. The analysis proceeds similar to that in Section 4. We first consider the optimal contracts for fixed exercise price, $w$, and then proceed with deriving the optimal $w$. The following proposition gives the solution for the supplier's optimal contract offer for fixed $w$.

Proposition B.1 For a given $w \geq 0$, in the optimal contract, the supplier offers the quantity $q^{*}(w)$ that solves the equation

$$
\begin{aligned}
& \int_{w-\theta}^{\bar{s}}\left(\phi s+\theta+\frac{r_{S}-r_{B}}{1+r_{B}}(s+\theta-w)\right) \bar{F}_{D}\left(q^{*}(w) \mid s\right) d F_{s}(s) \\
&+\frac{r_{S}-r_{B}}{1+r_{B}} \int_{\frac{w}{1-\phi}}^{\bar{s}}(s(1-\phi)-w) F_{D}\left(q^{*}(w) \mid s\right) d F_{s}(s)=\left(1+r_{S}\right) \beta-E[s](1-\phi),
\end{aligned}
$$

to the buyer at the price

$$
R(w)=\frac{1}{1+r_{B}}\left(E_{D, s}\left[(s+\theta-w)^{+} \min \left(D, q^{*}(w)\right)\right]+E_{D, s}\left[(s(1-\phi)-w)^{+}\left(q^{*}(w)-D\right)^{+}\right]\right) .
$$

Proof: The proof proceeds similar to that of Proposition 1. The buyer's expected profit can be written as

$$
\begin{aligned}
\pi_{B}(w, \theta)=-R(w)+\frac{1}{1+r_{B}} \int_{\underline{s}}^{\bar{s}}(p-s-\theta) E_{D}[D \mid s] f_{s}(s) d s \\
\quad+\frac{1}{1+r_{B}} \int_{w-\hat{\theta}}^{\bar{s}} \int_{\underline{D}}^{\bar{D}}(s+\theta-w) \min (x, q) f_{D, s}(x, s) d x d s \\
\quad+\frac{1}{1+r_{B}} \int_{\frac{w}{1-\phi}}^{\bar{s}} \int_{\underline{D}}^{\bar{D}}(s(1-\phi)-w)(q-x)^{+} f_{D, s}(x, s) d x d s
\end{aligned}
$$

As before, we can write the buyer's net benefit from entering the options contract as

$$
\hat{\pi}_{B}(q, w)=\pi_{B}(q, w)-\frac{1}{1+r_{B}} \int_{\underline{s}}^{\bar{s}}(p-s-\theta) E_{D}[D \mid s] f_{s}(s) d s
$$


Therefore,

$$
\begin{aligned}
\hat{\pi}_{B}(q, w)=-R(w)+\frac{1}{1+r_{B}} \int_{w-\theta}^{\bar{s}} \int_{\underline{D}}^{\bar{D}}(s+\theta-w) \min (x, q) f_{D, s}(x, s) d x d s & \\
& +\frac{1}{1+r_{B}} \int_{\frac{w}{1-\phi}}^{\bar{s}} \int_{\underline{D}}^{\bar{D}}(s(1-\phi)-w)(q-x)^{+} f_{D, s}(x, s) d x d s .
\end{aligned}
$$

Let us again define $\varphi(q, w)=\hat{\pi}_{B}(q, w)+R(w)$. The seller's problem formulation is the same as in (6), with the seller's expected profit now being

$$
\begin{aligned}
\pi_{S}(q, w)= & R(w)+\frac{1}{1+r_{S}}\left(\left(E[s](1-\phi)-\left(1+r_{S}\right) \beta\right) q\right. \\
& \left.+\int_{w-\theta}^{\frac{w}{1-\phi}} \int_{\underline{D}}^{\bar{D}}(w-s(1-\phi)) \min (x, q) f_{D, s}(x, s) d x d s+\int_{\frac{w}{1-\phi}}^{\bar{s}}(w-s(1-\phi)) q f_{s}(s) d s\right) .
\end{aligned}
$$

Define $V(q, w)=\pi_{S}(q, w)-R(w)$ as before. The solution to the supplier's problem proceeds with identical steps to that as given in the proof of Proposition 1. Knowing the buyer's type $\theta$, the seller can charge for the contract up to the point where the buyer's IR constraint is binding and satisfy his IC constraint with a single offer contract. As a consequence in the optimal contract, $R(q, w)=\varphi(q, w)$. Substituting this into the seller's objective and temporarily ignoring the seller's IR constraint, we have the unconstrained optimization problem $\max _{q}\{\varphi(q, w)+V(q, w)\}$, where

$$
\begin{aligned}
\varphi(q, w)+V(q, w)= & \frac{1}{1+r_{B}}\left(E_{D, s}\left[(s+\theta-w)^{+} \min (D, q)\right]+E_{D, s}\left[(s(1-\phi)-w)^{+}(q-D)^{+}\right]\right) \\
& +\frac{1}{1+r_{S}}\left(\left(E[s](1-\phi)-\beta-E_{s}\left[(s(1-\phi)-w)^{+}\right]\right) q\right. \\
& \left.\quad+\int_{w-\theta}^{\frac{w}{1-\phi}} \int_{\underline{D}}^{\bar{D}}(w-s(1-\phi)) \min (x, q) f_{D, s}(x, s) d x d s\right) \\
=\frac{1}{1+r_{S}}((E[s](1-\phi)-\beta) q+ & \int_{w-\theta}^{\bar{s}} \int_{\underline{D}}^{\bar{D}}(\phi s+\theta) \min (x, q) f_{D, s}(x, s) d x d s \\
& \left.+\frac{r_{S}-r_{B}}{\left(1+r_{B}\right)}\left(E_{D, s}\left[(s+\theta-w)^{+} \min (D, q)+(s(1-\phi)-w)^{+}(q-D)^{+}\right]\right)\right) .
\end{aligned}
$$

The first order condition is

$$
\begin{aligned}
\frac{d(\varphi(q, w)+V(q, w))}{d q}= & \frac{1}{1+r_{S}}\left(E[s](1-\phi)-\left(1+r_{B}\right) \beta+\int_{w-\theta}^{\bar{s}}(\phi s+\theta) \bar{F}_{D}(q \mid s) d F_{s}(s)\right) \\
& +\frac{r_{S}-r_{B}}{\left(1+r_{S}\right)\left(1+r_{B}\right)}\left(\int_{w-\theta}^{\bar{s}}(s+\theta-w) \bar{F}_{D}(q \mid s) d F_{s}(s)\right. \\
& \left.\quad+\int_{\frac{w}{1-\phi}}^{\bar{s}}(s(1-\phi)-w) \bar{F}_{D}(q \mid s) d F_{s}(s)\right)=0 .
\end{aligned}
$$


The second derivative is

$$
\begin{aligned}
\frac{d^{2}(\varphi(q, w)+V(q, w))}{d q^{2}}= & -\frac{1}{1+r_{S}} \int_{w-\theta}^{\bar{s}}(\phi s+\theta) f_{D \mid s}(q \mid s) d F_{s}(s) \\
& -\frac{r_{S}-r_{B}}{\left(1+r_{S}\right)\left(1+r_{B}\right)}\left(\int_{w-\theta}^{\frac{w}{1-\phi}}(s+\theta-w) f_{D \mid s}(q \mid s) d F_{s}(s)\right. \\
& \left.\quad+\int_{\frac{w}{1-\phi}}^{\bar{s}}(\phi s+\theta) f_{D \mid s}(q \mid s) d F_{s}(s)\right)
\end{aligned}
$$

Note that since $\left(r_{S}-r_{B}\right) /\left(1+r_{S}\right)\left(1+r_{B}\right) \geq-1 /\left(1+r_{S}\right)$, we have

$$
\frac{d^{2}(\varphi(q, w)+V(q, w))}{d q^{2}} \leq-\frac{1}{1+r_{S}} \int_{w-\theta}^{\frac{w}{1-\phi}}(w-s(1-\phi)) f_{D \mid s}(q \mid s) d F_{s}(s)<0 .
$$

Therefore, the solution of (B.8) maximizes $\varphi(q, w)+V(q, w)$. Further, since $q=0$ is feasible and yields the seller zero expected profit, and, by (B.10), $\varphi(q, w)+V(q, w)$ is strictly concave, the IR constraint of the seller is satisfied. Hence $q^{*}$ that solves (B.1) is the seller's optimal contract quantity offer. The optimal reservation price for this quantity can be calculated by plugging $q^{*}$ in $\varphi(q, w)$ since the buyer's IR constraint is binding, i.e., $R\left(q^{*}, w\right)=\varphi\left(q^{*}, w\right)$. This completes the proof.

We can now present the solution for the optimization of the exercise price $w$.

\section{Proposition B.2}

(i) When $r_{B}<r_{S}$, sales contracts are optimal, i.e. $w^{*}=0$.

(ii) When $r_{B}=r_{S}$, there is a continuum of optimal contracts. In particular, any $w^{*} \in[0, \underline{s}+\theta]$ is optimal, hence, both sales and options contracts can optimally emerge.

(iii) When $r_{B}>r_{S}$, options contracts are optimal. In particular the optimal exercise price is $w^{*} \in$ $[\underline{s}+\theta, \bar{s}+\theta]$.

Proof: The proof again proceeds similar to its counterpart in Section 4, i.e., the proof of part (ii) of Proposition 1. From the proof of Proposition B.1, we know that $\pi_{S}(w)=\varphi\left(q^{*}(w), w\right)+V\left(q^{*}(w), w\right)$. Then, by taking the total derivative of (B.7) with respect to $w$, we have

$$
\begin{aligned}
\frac{d \pi_{S}(w)}{d w}= & \frac{\partial \pi_{S}(w)}{\partial w}+\frac{d \pi_{S}(w)}{d q^{*}(w)} \cdot \frac{d q^{*}(w)}{d w} \\
= & -\frac{1}{1+r_{S}}(\phi(w-\theta)+\theta) E_{D}\left[\min \left(D, q^{*}(w)\right) \mid s=w-\theta\right] \cdot f_{s}(w-\theta) \\
& -\frac{r_{S}-r_{B}}{\left(1+r_{S}\right)\left(1+r_{B}\right)}\left(\int_{w-\theta}^{\bar{s}} E_{D}\left[\min \left(D, q^{*}(w)\right) \mid s\right] d F_{s}(s)\right. \\
& \left.+\int_{\frac{w}{1-\phi}}^{\bar{s}} E_{D}\left[\left(q^{*}(w)-D\right)^{+} \mid s\right] d F_{s}(s)\right),
\end{aligned}
$$


where $q^{*}(w)$ is solved by (B.1).

Now, first, consider the case where $r_{B}<r_{S}$. In this case, all terms in (A.5) are negative for all $w \geq 0$. Therefore, the optimal $w$ for the buyer is 0 and optimal contracts are sales contracts. Now suppose $r_{B}=r_{S}$. In this case $\frac{d \pi_{S}(w)}{d w}=-\frac{1}{1+r_{S}}(\phi(w-\theta)+\theta) E_{D}\left[\min \left(D, q^{*}(w)\right)\right] \cdot f_{s}(w-\theta)<0$ for all $w>\underline{s}+\theta$, and $\frac{d \pi_{S}(w)}{d w}=0$ otherwise. Therefore, there is a continuum of optimal contracts, for $w^{*} \in[0, \underline{s}+\theta]$, and both sales $\left(w^{*}=0\right)$ and options contracts $\left(w^{*}>0\right)$ can prevail. Finally, when $r_{B}>r_{S}$, on $w \in[0, \underline{s}+\theta]$, the second term in (A.5) is strictly positive, while the first term is zero. Therefore $\pi_{S}(w)$ is strictly increasing on $w \in[0, \underline{s}+\theta]$. Note that for $w>\bar{s}+\theta, d \pi_{S}(w) / d w=0$. Combining these with the continuity of $p i_{S}(w)$, it follows that $\pi_{S}(w)$ is maximized $w \in[\underline{s}+\theta, \bar{s}+\theta]$. This completes the proof.

\section{The Analysis of the Case with Perfect Correlation between Spot Price and Consumer Demand Distributions for Random Buyer Type}

In this section, we analyze the case of perfect correlation between the spot price and consumer demand and demonstrate that the main conclusions and insights are preserved. We start with a perfect positive correlation case, and then study the perfect negative correlation. We show that in both cases the main conclusions are preserved. For both the positive and negative correlation cases, we will focus on the analysis for $K \geq \bar{D}$.

\section{C.1 Perfect Positive Correlation between Spot Price and Demand Distributions}

Suppose

$$
D=\underline{D}+\frac{\bar{D}-\underline{D}}{\bar{s}-\underline{s}}(s-\underline{s})
$$

Define $\alpha_{0}=\underline{D}-((\bar{D}-\underline{D}) /(\bar{s}-\underline{s})) \underline{s}$ and $\alpha_{1}=((\bar{D}-\underline{D}) /(\bar{s}-\underline{s}))$. That is $D=\alpha_{0}+\alpha_{1} s$. The following proposition, which is parallel to Proposition 2 in Section 5 gives the optimal contracting outcome for a given exercise price $w \geq 0$.

\section{Proposition C.1}

(i) If the flat price conditions as defined in Section 5 are satisfied, the optimal contracts are not volumedependent. Rather, in the optimal offer, the reservation price is constant and given by $\varphi(K, w, \underline{\theta}) /(1+$ $\left.r_{B}\right)$, and $q^{*}(w, \theta)=K$ for all $\theta \in[\underline{\theta}, \bar{\theta}]$.

(ii) Suppose the flat price conditions are not satisfied. Then given $0<\rho<\bar{\rho}$, where $\bar{\rho}$ is as defined in Proposition 2, the optimal reservation price schedule for the seller is volume-dependent. Specifically, 
the optimal quantity ordered for type $\theta$ buyer is

$$
q^{*}(w, \theta)=\alpha_{0}+\alpha_{1} \bar{F}_{s}^{-1}\left(\frac{\left(\left(1+r_{S}\right) \beta-E[s](1-\phi)\right)^{+}-(\rho-1) G(w)}{\eta(w, \theta)-(\rho-1) G(w)}\right),
$$

where $\eta$ is as defined in Proposition 2 and $G$ is as defined in Lemma 1. The optimal total reservation fee paid by a type $\theta$ buyer is is again as given in (16).

Proof: The proof will follow the parallel steps to Proposition 2. We can write the type $\theta$ buyer's expected profit from purchasing $q$ options from the seller at exercise price $w$ as

$$
\begin{aligned}
\pi_{B}(q, w, \theta) & =-R(w, q)+\frac{1}{1+r_{B}}\left\{\int_{\underline{s}}^{\bar{s}}(p-s-\theta)\left(\alpha_{0}+\alpha_{1} s\right) d F_{s}(s)\right. \\
& \left.+\int_{w-\theta}^{\bar{s}}(s+\theta-w) \min \left\{\alpha_{0}+\alpha_{1} s, q\right\} d F_{s}(s)+\int_{w /(1-\phi)}^{\bar{s}}(s(1-\phi)-w)\left(q-\alpha_{0}-\alpha_{1} s\right)^{+} d F_{s}(s)\right\} \\
& =-R(w, q)+\frac{1}{1+r_{B}}\left\{\alpha_{0}(p-\theta)-\left(\alpha_{0}-\alpha_{1}(p-\theta)\right)-\left(\alpha_{0}-\alpha_{1}(p-\theta)\right) E[s]-\alpha_{1} E\left[s^{2}\right]\right. \\
& +\int_{w-\theta}^{\bar{s}}(s+\theta-w)\left(\alpha_{0}+\alpha_{1} \min \left\{s, \frac{q-\alpha_{0}}{\alpha_{1}}\right\}\right) d F_{s}(s) \\
& \left.+\int_{w /(1-\phi)}^{\bar{s}}(s(1-\phi)-w)\left(q-\alpha_{0}-\alpha_{1} \min \left\{s, \frac{q-\alpha_{0}}{\alpha_{1}}\right\}\right) d F_{s}(s)\right\} .
\end{aligned}
$$

Similar to Section 4, define

$$
\hat{\pi}_{B}(q, w, \theta)=\pi_{B}(q, w, \theta)-\frac{1}{1+r_{B}}\left(\alpha_{0}(p-\theta)-\left(\alpha_{0}-\alpha_{1}(p-\theta)\right) E[s]-\alpha_{1} E\left[s^{2}\right]\right),
$$

as the buyer's net benefit from purchasing option contracts. Then,

$$
\begin{aligned}
\hat{\pi}_{B}(q, w, \theta)= & -R(w, q)+\frac{1}{1+r_{B}} \int_{w-\theta}^{\bar{s}}(s+\theta-w)\left(\alpha_{0}+\alpha_{1} \min \left\{s, \frac{q-\alpha_{0}}{\alpha_{1}}\right\}\right) d F_{S}(s) \\
& +\frac{1}{1+r_{B}} \int_{w /(1-\phi)}^{\bar{s}}(s(1-\phi)-w)\left(q-\alpha_{0}-\alpha_{1} \min \left(s, \frac{q-\alpha_{0}}{\alpha_{1}}\right)\right) d F_{s}(s) .
\end{aligned}
$$

Define $\varphi(q, w, \theta)=\left(1+r_{B}\right)\left(\hat{\pi}_{B}(q, w, \theta)+R(w, q)\right)$.

The seller's profit from contracting $q$ options at exercise price $w$ with a type $\theta$ buyer can then be written 
as $\pi_{S}(q, w, \theta)=R(q, w)+V(q, w, \theta)$, where

$$
\begin{aligned}
V(q, w, \theta)=-\beta q+\frac{1}{1+r_{S}} & \left\{\int_{\underline{s}}^{w-\theta} s(1-\phi) d F_{s}(s) q+w q \bar{F}_{s}\left(\frac{w}{1-\phi}\right)\right. \\
& \left.+\int_{w-\theta}^{w /(1-\phi)}\left(w \min \left\{\alpha_{0}+\alpha_{1} s, q\right\}+s(1-\phi) E\left[\left(q-\alpha_{0}-\alpha_{1} s\right)^{+}\right] d F_{s}(s)\right)\right\} \\
& +\left(\frac{E[s](1-\phi)}{1+r_{S}}-\beta\right)^{+} \cdot(K-q) \\
=-\beta q+\frac{1}{1+r_{S}} & \left\{\left(E[s](1-\phi)-E\left[(s(1-\phi)-w)^{+}\right]\right) q\right. \\
& +\int_{w-\theta}^{w /(1-\phi)}\left(w-s(1-\phi)\left(\alpha_{0}+\alpha_{1} \min \left(s, \frac{q-\alpha_{0}}{\alpha_{1}}\right)\right) d F_{s}(s)\right\} \\
& +\left(\frac{E[s](1-\phi)}{1+r_{S}}-\beta\right)^{+} \cdot(K-q) .
\end{aligned}
$$

Now, as we have shown in the proof of Proposition 2, the seller's expected profit can be written as

$$
\pi_{S}(w)=\int_{\underline{\theta}}^{\bar{\theta}} H(q(w, \theta), w, \theta) d F_{\theta}(\theta),
$$

where

$$
H(q(w, \theta), w, \theta)=V(q(w, \theta), w, \theta)+\frac{1}{1+r_{B}}\left\{\varphi(q(w, \theta), w, \theta)-g_{\theta}(\theta) \frac{\partial \varphi(q(w, \theta), w(\theta))}{\partial \theta}\right\}
$$

From (C.5) and (C.6), we have

$$
\begin{aligned}
& H(q(w, \theta), w, \theta)= \frac{1}{1+r_{S}}\left\{\left(E[s](1-\phi)-\left(1+r_{S}\right) \beta\right) q\right. \\
& \quad+\int_{w-\theta}^{\bar{s}}\left(\phi s+\theta-g_{\theta}(\theta)\right)\left(\alpha_{0}+\alpha_{1} \min \left\{s, \frac{q-\alpha_{0}}{\alpha_{1}}\right\} d F_{s}(s)\right\} \\
& \quad+\frac{r_{S}-r_{B}}{\left(1+r_{S}\right)\left(1+r_{B}\right)}\left\{\int_{w-\theta}^{\bar{s}}\left(s+\theta-g_{\theta}(\theta)-w\right)\left(\alpha_{0}+\alpha_{1} \min \left\{s, \frac{q-\alpha_{0}}{\alpha_{1}}\right\}\right) d F_{s}(s)\right. \\
&\left.+\int_{w /(1-\phi)}(s(1-\phi)-w)\left(q-\alpha_{0}-\alpha_{1} s\right)^{+} d F_{s}(s)\right\}+\left(\frac{E[s](1-\phi)}{1+r_{S}}-\beta\right)^{+} \cdot(K-q)(. \mathrm{C} .9
\end{aligned}
$$

Again as in the proof of Proposition 2, (C.7) can be optimized pointwise. By (C.9), writing the first and second derivatives

$$
\begin{aligned}
\frac{d H(q(w, \theta), w, \theta)}{d q(w, \theta)}= & \frac{1}{1+r_{S}}\left\{\left(\left(1+r_{S}\right) \beta-E[s](1-\phi)\right)^{+}+\bar{F}_{s}\left(\frac{q-\alpha_{0}}{\alpha_{1}}\right) \int_{w-\theta}^{\bar{s}}\left(\phi s+\theta-g_{\theta}(\theta)\right) d F_{s}(s)\right\} \\
+ & \frac{r_{S}-r_{B}}{\left(1+r_{S}\right)\left(1+r_{B}\right)}\left\{\bar{F}_{s}\left(\frac{q-\alpha_{0}}{\alpha_{1}}\right) \int_{w-\theta}^{\bar{s}}\left(s+\theta-g_{\theta}(\theta)(\theta)-w\right) d F_{s}(s)\right. \\
& \left.+F_{s}\left(\frac{q-\alpha_{0}}{\alpha_{1}}\right) \int_{w /(1-\phi)}^{\bar{s}}(s(1-\phi)-w) d F_{s}(s)\right\},
\end{aligned}
$$


and

$$
\frac{d^{2} H(q(w(\theta), w, \theta))}{d q^{2}(w, \theta)}=-\frac{f_{s}\left(\frac{q-\alpha_{0}}{\alpha_{1}}\right)}{\alpha_{1}\left(1+r_{S}\right)}(\eta(w, \theta)-(\rho-1) G(w)) .
$$

As we have shown in the proof of Proposition 2, $\eta(w, \theta)-(\rho-1) G(w) \geq 0$. Therefore $\left(d^{2} H(q(w(\theta), w, \theta))\right) /\left(d q^{2}(w, \theta)\right) \leq 0$, and the first order condition is sufficient for pointwise optimality of (C.7) for all $\theta \in[\underline{\theta}, \bar{\theta}]$. Hence, equating (C.10) to zero and solving for $q$, we obtain

$$
q^{*}(w, \theta)=\alpha_{0}+\alpha_{1} \bar{F}_{s}^{-1}\left(\frac{\left(\left(1+r_{S}\right) \beta-E[s](1-\phi)\right)^{+}-(\rho-1) G(w)}{\eta(w, \theta)-(\rho-1) G(w)}\right) .
$$

Therefore, similar to Proposition 2, and by Lemma 1, $q^{*}$ will be constant if the flat-price conditions are satisfied, and will be volume dependent otherwise. The rest of the proof follows the same steps in the proof of Proposition 2.

We next explore the seller's optimization of the exercise price $w$. The following proposition presents the result.

\section{Proposition C.2}

(i) If $r_{S}>r_{B}, w^{*}=0$. That is, selling the intermediate good is optimal for the supplier, rather than offering options to the buyer.

(ii) If $r_{S}=r_{B}$, the seller's profit is maximized by setting any exercise price $w^{*}$, where $0 \leq w^{*} \leq \underline{s}+\underline{\theta}$.

(iii) If $r_{S}<r_{B}, \underline{s}+\underline{\theta}<w^{*} \leq \bar{s}+\bar{\theta}$, i.e., the seller prefers offering options to selling the intermediate good.

Proof: The proof will proceed following steps parallel to those in the proof of Proposition 6. By (C.7) and using the envelope theorem,

$$
\frac{d \Pi_{S}^{*}(w)}{d w}=\int_{\underline{\theta}}^{\bar{\theta}} \frac{\partial H\left(q^{*}(w, \theta), w, \theta\right)}{\partial w} d F_{\theta}(\theta) .
$$


Therefore,

$$
\begin{aligned}
\frac{d \Pi_{S}^{*}(w)}{d w} & =\frac{1}{1+r_{S}} \int_{\underline{\theta}}^{\bar{\theta}}\left\{-\left(\phi(w-\theta)+\theta-g_{\theta}(\theta)(t)\right)\left(\alpha_{0}+\alpha_{1} \min \left\{w-\theta, \frac{q-\alpha_{0}}{\alpha_{1}}\right\}\right) f_{s}(w-\theta)\right. \\
& -(\rho-1)\left(\int_{w-\theta}^{\bar{s}}\left(\alpha_{0}+\alpha_{1} \min \left\{s, \frac{q-\alpha_{0}}{\alpha 1}\right\}\right) d F_{s}(s)-g_{\theta}(\theta)\left(\alpha_{0}+\alpha_{1} \min \left\{w-\theta, \frac{q-\alpha_{0}}{\alpha_{1}}\right\}\right) f_{s}(w-\theta)\right. \\
& \left.\left.\int_{w /(1-\phi)}^{\bar{s}}\left(q-\alpha_{0}-\alpha_{1} s\right)^{+} d F_{s}(s)\right)\right\} d F_{\theta}(\theta) \\
& =\int_{\underline{\theta}}^{\bar{\theta}}\left\{-\left(\phi(w-\theta)+\theta-\rho g_{\theta}(\theta)\right)\left(\alpha_{0}+\alpha_{1} \min \left\{w-\theta, \frac{q-\alpha_{0}}{\alpha_{1}}\right\}\right) f_{s}(w-\theta)\right. \\
& \left.-(\rho-1)\left(\int_{w-\theta}^{\bar{s}}\left(\alpha_{0}+\alpha_{1} \min \left\{s, \frac{q-\alpha_{0}}{\alpha_{1}}\right\}\right) d F_{s}(s)+\int_{\frac{w}{1-\phi}}^{\bar{s}}\left(q-\alpha_{0}-\alpha_{1} s\right)^{+} d F_{s}(s)\right)\right\} d F_{\theta}(\theta) .(\mathrm{C} .14)
\end{aligned}
$$

Now, for any given $\theta \in[\underline{\theta}, \bar{\theta}]$,

$$
\frac{\partial H\left(q^{*}(w, \theta), w, \theta\right)}{\partial w}= \begin{cases}-(\rho-1)\left(q^{*}(w, \theta)-\int_{\underline{s}}^{w /(1-\phi)}\left(q-\alpha_{0}-\alpha_{1} s\right)^{+} d F_{s}(s)\right) & \text { for } w<\underline{s}+\theta ; \\ -f_{s}(w-\theta) \nu(w, \theta)\left(\alpha_{0}+\alpha_{1} \min \left\{w-\theta, \frac{q-\alpha_{0}}{\alpha_{1}}\right\}\right) & \\ -(\rho-1)\left(\int_{w-\theta}^{\bar{s}}\left(\min \left\{s, \frac{q-\alpha_{0}}{\alpha_{1}}\right\}-\min \left\{w-\theta, \frac{q-\alpha_{0}}{\alpha_{1}}\right\}\right) d F_{s}(s)\right. & \\ \left.\quad+\int_{\frac{w}{1-\phi}}^{\bar{s}}\left(q-\alpha_{0}-\alpha_{1} s\right)^{+} d F_{s}(s)\right) & \text { for } w \in[\underline{s}+\theta, \bar{s}+\theta] ; \\ 0 & \text { for } w>\bar{s}+\theta .\end{cases}
$$

Substituting (C.15) in (C.14), we then have

$$
\frac{d \pi_{S}^{*}(w)}{d w}=-(\rho-1) \int_{\underline{\theta}}^{\bar{\theta}}\left(q^{*}(w, \theta)-\int_{\underline{s}}^{w /(1-\phi)}\left(q-\alpha_{0}-\alpha_{1} s\right)^{+} d F_{s}(s)\right) d F_{\theta}(\theta),
$$

for $w<\underline{s}+\underline{\theta}$, and

$$
\begin{aligned}
\frac{d \pi_{S}^{*}(w)}{d w}= & -\int_{\underline{\theta}}^{\tilde{\theta}}\left\{f_{s}(w-\theta) \nu(w, \theta)\left(\alpha_{0}+\alpha_{1} \min \left\{w-\theta, \frac{q-\alpha_{0}}{\alpha_{1}}\right\}\right)\right. \\
& +(\rho-1)\left(\int_{w-\theta}^{\bar{s}}\left(\min \left\{s, \frac{q-\alpha_{0}}{\alpha_{1}}\right\}-\min \left\{w-\theta, \frac{q-\alpha_{0}}{\alpha_{1}}\right\}\right) d F_{s}(s)\right. \\
& \left.\left.\quad+\int_{\frac{w}{1-\phi}}^{\bar{s}}\left(q-\alpha_{0}-\alpha_{1} s\right)^{+} d F_{s}(s)\right)\right\} d F_{\theta}(\theta) \\
& -(\rho-1) \int_{\underline{\theta}}^{\bar{\theta}}\left(q^{*}(w, \theta)-\int_{\underline{s}}^{w /(1-\phi)}\left(q-\alpha_{0}-\alpha_{1} s\right)^{+} d F_{s}(s)\right) d F_{\theta}(\theta),
\end{aligned}
$$

for any $w=\underline{s}+\tilde{\theta}$, and $\tilde{\theta} \in[\underline{\theta}, \bar{\theta}]$, and where $\nu(w, \theta)$ is as defined in the proof of Proposition 2. As shown in the proof of Proposition $2 \nu(w, \theta) \geq 0, \forall w \geq \underline{s}+\theta$; and by (C.15), $d \pi_{S}^{*}(w) / d w \leq 0$ for all $w \geq \underline{s}+\bar{\theta}$. When $\rho>1$, since the integrand in (C.16) is positive for all $\theta, d \pi_{S}^{*}(w) / d w<0$ for $0 \leq w<\underline{s}+\underline{\theta}$. Similarly, since both terms in (C.17) are negative, $d \pi_{S}^{*}(w) / d w<0$ also follows for $\underline{s}+\underline{\theta} \leq w \leq \bar{s}+\bar{\theta}$. Therefore, 
similar to Proposition 6 , for $\rho>1, \pi_{S}^{*}(w)$ is maximized at $w^{*}=0$, which proves part $(i)$. When $\rho=1$, by (C.16), $d \pi_{S}^{*}(w) / d w=0$ for $0 \leq w<\underline{s}+\underline{\theta}$, and by $(\mathrm{C} .17), d \pi_{S}^{*}(w) / d w<0$ for $\underline{s}+\underline{\theta} \leq w \leq \bar{s}+\bar{\theta}$. Hence $\pi_{S}^{*}(w)$ is flat on $w \in[0, \underline{s}+\underline{\theta}]$, strictly decreasing on $[\underline{s}+\underline{\theta}, \bar{s}+\bar{\theta}]$, and flat again for $w>\bar{s}+\bar{\theta}$, and thus is again maximized for any $w \in[0, \underline{s}+\underline{\theta}]$, as stated in part (ii). Finally, again similar to Proposition 6 , when $\rho<1$, by $\left(\right.$ C.16), $d \pi_{S}^{*}(w) / d w>0$ for $0 \leq w<\underline{s}+\underline{\theta}$. Further, by $\left(\right.$ C.17), $d \pi_{S}^{*}(w) / d w>0$ for $w=\underline{s}+\underline{\theta}$, and $\pi_{S}^{*}(w)$ is non-increasing for $w>\bar{s}+\bar{\theta}$. Since $\pi_{S}^{*}(w)$ is continuous in $w$, it follows that for $\rho<1, \pi_{S}^{*}(w)$ is maximized at a $w^{*} \in[\underline{s}+\underline{\theta}, \bar{s}+\bar{\theta}]$ as stated in part (iii) of the proposition. This completes the proof.

\section{C.2 Perfect Negative Correlation between Spot Price and Demand Distributions}

Now suppose

$$
D=\underline{D}-\frac{\bar{D}-\underline{D}}{\bar{s}-\underline{s}}(s-\underline{s}) .
$$

The analysis will closely follow the analysis for the positive correlation case. Define $\alpha_{0}=\bar{D}+((\bar{D}-\underline{D}) /(\bar{s}-$ $\underline{s})) \underline{s}$ and $\alpha_{1}=((\bar{D}-\underline{D}) /(\bar{s}-\underline{s}))$. That is, $D=\alpha_{0}-\alpha_{1} s$.

\section{Proposition C.3}

(i) If the flat price conditions as defined in Section 5 are satisfied, the optimal contracts are not volumedependent. Rather, in the optimal offer, the reservation price is constant and given by $\varphi(K, w, \underline{\theta}) /(1+$ $\left.r_{B}\right)$, and $q^{*}(w, \theta)=K$ for all $\theta \in[\underline{\theta}, \bar{\theta}]$.

(ii) Suppose the flat price conditions are not satisfied. Then given $0<\rho<\bar{\rho}$, where $\bar{\rho}$ is as defined in Proposition 2, the optimal reservation price schedule for the seller is volume-dependent. Specifically, the optimal quantity ordered for type $\theta$ buyer is

$$
q^{*}(w, \theta)=\alpha_{0}-\alpha_{1} F_{s}^{-1}\left(\frac{\left(\left(1+r_{S}\right) \beta-E[s](1-\phi)\right)^{+}-(\rho-1) G(w)}{\eta(w, \theta)-(\rho-1) G(w)}\right)
$$

where $\eta$ is as defined in Proposition 2 and $G$ is as defined in Lemma 1. The optimal total reservation fee paid by a type $\theta$ buyer is is again as given in (16).

Proof: The proof is very similar to that of Proposition C.1. Therefore, we will just provide a sketch of it highlighting the differences. Evaluating a type $\theta$ buyer's net profit from purchasing $q$ options at the exercise price $w \geq 0$, we obtain

$$
\begin{aligned}
\hat{\pi}_{B}(q, w, \theta)=-R(w, q)+ & \frac{1}{1+r_{B}} \int_{w-\theta}^{\bar{s}}(s+\theta-w)\left(\alpha_{0}-\alpha_{1} \max \left\{s, \frac{\alpha_{0}-q}{\alpha_{1}}\right\}\right) d F_{S}(s) \\
& +\frac{1}{1+r_{B}} \int_{w /(1-\phi)}^{\bar{s}}(s(1-\phi)-w)\left(\alpha_{1} s-\alpha_{0}+q\right)^{+} d F_{s}(s),
\end{aligned}
$$


while the seller's expected profit from this transaction can be written as $\pi_{S}(q, w, \theta)=R(q, w)+V(q, w, \theta)$, where

$$
\begin{aligned}
V(q, w, \theta)=-\beta q+\frac{1}{1+r_{S}} & \left\{\left(E[s](1-\phi)-E\left[(s(1-\phi)-w)^{+}\right]\right) q\right. \\
+ & \int_{w-\theta}^{w /(1-\phi)}\left(w-s(1-\phi)\left(\alpha_{0}-\alpha_{1} \max \left(s, \frac{q-\alpha_{0}}{\alpha_{1}}\right)\right) d F_{s}(s)\right\} \\
+ & \left(\frac{E[s](1-\phi)}{1+r_{S}}-\beta\right)^{+} \cdot(K-q) .
\end{aligned}
$$

Once again, the seller's expected profit can be written as (C.7), where $H(q(w, \theta), w, \theta)$ is as defined in (C.8). Substituting $D=\alpha_{0}-\alpha_{1} s$, we then obtain

$$
\begin{aligned}
& H(q(w, \theta), w, \theta)= \frac{1}{1+r_{S}}\left\{\left(E[s](1-\phi)-\left(1+r_{S}\right) \beta\right) q\right. \\
& \quad+\int_{w-\theta}^{\bar{s}}\left(\phi s+\theta-g_{\theta}(\theta)\right)\left(\alpha_{0}-\alpha_{1} \max \left\{s, \frac{q-\alpha_{0}}{\alpha_{1}}\right\} d F_{s}(s)\right\} \\
&+\frac{r_{S}-r_{B}}{\left(1+r_{S}\right)\left(1+r_{B}\right)}\left\{\int_{w-\theta}^{\bar{s}}\left(s+\theta-g_{\theta}(\theta)-w\right)\left(\alpha_{0}-\alpha_{1} \max \left\{s, \frac{q-\alpha_{0}}{\alpha_{1}}\right\}\right) d F_{s}(s)\right. \\
&\left.\quad+\int_{w /(1-\phi)}(s(1-\phi)-w)\left(q-\alpha_{0}+\alpha_{1} s\right)^{+} d F_{s}(s)\right\} \\
&+\left(\frac{E[s](1-\phi)}{1+r_{S}}-\beta\right)^{+} \cdot(K-q) .
\end{aligned}
$$

Again $\Pi_{S}(w)$ can be optimized pointwise. By (C.22), writing the first and second derivatives

$$
\begin{aligned}
\frac{d H(q(w, \theta), w, \theta)}{d q(w, \theta)}= & \frac{1}{1+r_{S}}\left\{\left(\left(1+r_{S}\right) \beta-E[s](1-\phi)\right)^{+}+F_{s}\left(\frac{\alpha_{0}-q}{\alpha_{1}}\right) \int_{w-\theta}^{\bar{s}}\left(\phi s+\theta-g_{\theta}(\theta)\right) d F_{s}(s)\right\} \\
+ & \frac{r_{S}-r_{B}}{\left(1+r_{S}\right)\left(1+r_{B}\right)}\left\{F_{s}\left(\frac{\alpha_{0}-q}{\alpha_{1}}\right) \int_{w-\theta}^{\bar{s}}\left(s+\theta-g_{\theta}(\theta)(\theta)-w\right) d F_{s}(s)\right. \\
& \left.+\bar{F}_{s}\left(\frac{\alpha_{0}-q}{\alpha_{1}}\right) \int_{w /(1-\phi)}^{\bar{s}}(s(1-\phi)-w) d F_{s}(s)\right\},
\end{aligned}
$$

and

$$
\frac{d^{2} H(q(w(\theta), w, \theta))}{d q^{2}(w, \theta)}=-\frac{f_{s}\left(\frac{\alpha_{0}-q}{\alpha_{1}}\right)}{\alpha_{1}\left(1+r_{S}\right)}(\eta(w, \theta)-(\rho-1) G(w)) \leq 0 .
$$

Therefore, again the first order condition is sufficient, and the optimal quantity for type $\theta$ can be found by equating (C.23) to zero. The rest of the proof follows similar to the proof of Proposition C.1.

Finally, for the optimization of the exercise price $(w)$, the statement and the results for the perfect positive correlation case, as stated in Proposition C.2, are identically preserved. The proof proceeds with closely parallel steps and hence is skipped. 